\title{
Myxomycetes of Russia: a history of research and a checklist of species
}

\author{
Bortnikov F.M. ." ${ }^{*}$ Matveev A.V. ${ }^{1}$, Gmoshinskiy V.I. ${ }^{1}$, \\ Novozhilov Yu.K. ${ }^{2}$, Zemlyanskaya I.V. ${ }^{3}$, Vlasenko A.V. ${ }^{4}$, \\ Schnittler M. ${ }^{5}$, Shchepin O.N. ${ }^{2}$, Fedorova N.A. ${ }^{2,6}$
}

1 Lomonosov Moscow State University, Faculty of Biology, Leninskie Gory 1-12, Moscow 119991, Russia

2 Komarov Botanical Institute of the Russian Academy of Sciences, Laboratory of Systematics and Geography of Fungi, Prof. Popov Street 2, St. Petersburg 197376, Russia

3 Volgograd State Medical University, Pavshikh Bortsov Square 1, Volgograd 400131, Russia ${ }^{4}$ Central Siberian Botanical Garden, Siberian Branch of the Russian Academy of Sciences,

Zolotodolinskaya, 101, Novosibirsk 630090, Russia

${ }^{5}$ Institute of Botany and Landscape Ecology, University of Greifswald, Soldmannstr. 15, Greifswald D-17487, Germany

6 Saint Petersburg State University, Universitetskaya emb. 7-9, 199034 St. Petersburg, Russia

* Corresponding author:

f.m.bortnikov@gmail.com

Keywords: Amoebozoa, bibliography, biodiversity, database, geographical distribution, literature review, Palearctic, slime molds

Article info:

Received: 06 August 2020

Accepted: 02 October 2020

Published online: 07 December 2020

Corresponding Editor: Nikki Heherson A. Dagamac

\begin{abstract}
The main objective of this paper is to provide an overview of the history and the level of research on biodiversity of myxomycetes in Russia. The first doubtless mention of myxomycetes in Russia dates back to the 18th century. Since then, numerous studies on myxomycete diversity in different regions of Russia have been published. Yu.K. Novozhilov summarized all accumulated data by publishing a list of 304 species in 2005. However, new data on species diversity, biogeography, and ecology of myxomycetes have been published in recent years. Recent research aims to fill this gap. This paper includes 321 sources, including studies published after 2005 and several works absent in previous reports. A full list of 455 myxomycete species found in Russia includes references to all literature sources. The analyzed database consists of more than 14600 records in the "publication-region-species" format. Additionally, our research includes a detailed historical overview of the myxomycete studies in Russia. We hope that our information system, also available online at https://russia.myxomycetes.org/, will create a solid foundation for future studies of myxomycete biodiversity in Russia, particularly in the understudied regions.
\end{abstract}




\section{Introduction}

An assessment of myxomycete species diversity in Russia is especially relevant due to its vast territory with various landscapes and terrestrial biomes such as tundra, taiga, broad-leaved forests, dry and wet subtropics, steppes, and deserts. It should be noted that myxomycete studies of these biomes in Russia are still restricted to a limited number of sites (Novozhilov 2005). However, many new data on species diversity, biogeography, and ecology of myxomycetes have been published in recent years. These studies are based on the herbarium collections of myxomycete fruiting bodies (sporocarps) collected over decades in the field and/or obtained using the moist chamber culture technique, as well as on data from metagenomic analyses, a method that has lately been used to reveal the hidden diversity of myxomycetes (Shchepin et al. 2017, 2019a,b).

Our study aims to analyze the myxomycete distribution in Russia based on all available bibliographic sources and compile an exhaustive list of species found in each region accompanied by a reference base of the sources used.

We did not aim to perform a full critical revision of all available literature sources on myxomycete biodiversity, since it would demand additional efforts. This list was compiled to aid scientists in searching for the required bibliographic sources, as well as to save some lesser-known works from "scientific oblivion". We also urge all researchers to refer to the indicated primary sources in order to avoid spreading possible mistakes that are almost inevitable in large biodiversity reviews like this.

\section{Myxomycete studies in Russia}

The earliest mention of myxomycetes in the territory of modern Russia might probably belong to the German botanist, Johannes Loeselius. His works considering plants of Prussia, which part has become the Kaliningrad region, included a brief description on the taxon Fungus non vescus XXXI
(Loeselius 1654, 1703), that might have actually been Lycogala epidendrum (Rostafiński 1874).

The first report of myxomycete collection in the territory of modern Russia in 18th century was made by Johann Christian Buxbaum (head of the Apothecary Garden in St. Petersburg), who worked at the Russian Academy of Sciences in 1721-1729 and provided the first fairly accurate description of the St. Petersburg Governorate flora. His work (Buxbaum 1740) included an illustration of Lycoperdon sanguineum sphaericum (Lycogala epidendrum (L.) Fr.), although the location was not indicated for this specimen. The next mention of myxomycetes was discovered in the manuscript of Stepan Krasheninnikov Flora ingrica, which was edited and published in 1761 by David de Gorter. In this publication, he noted two species: Mucor embolus L. (presumably a species of Arcyria or Stemonitis) and Lycoperdon epidendrum L. (Lycogala epidendrum (L.) Fr.), collected in the vicinity of St. Petersburg (Gorter 1761). In 1799, the Russian botanist Grigory Fedorovich Sobolewski published Flora Petropolitana, where he reported 13 myxomycete species for the Petersburg Governorate, although it is impossible to provide reliable modern synonyms for six of these species (Sobolewski 1799, 1802; the second publication includes the same material, but in Russian). There are only a few reports of myxomycetes in 18th-century floristic publications (Pallas 1771, 1773, 1788, 1794; Falk 1786; Georgi 1790, 1800; Stephan 1792). These studies can be referred to as a starting point for the study of myxomycete diversity in Russia.

In the 19th and early 20th centuries, at least 30 publications included data on myxomycetes. These studies covered the vicinity of Moscow (Martius 1817; Bucholtz 1897; Heyden 1899; Hennings 1903, 1904, 1906; Dokturovskiy 1905; Mosolov 1906) and St. Petersburg (Weinmann 1836; Rosanoff 1868; Rostafiński 1874, 1875; Grimm 1896), as well as Smolensk (Jaczewski 1893, 1895), Kaluga (Rostafiðski 1874), Chernigov ${ }^{1}$ (Borščow 1869), Novgorod (Tranzschel 1901), Ufa (Schell 1883) and Kazan Governorates (Shiliakow 1889), North Caucasus (Hollós 1902, 1905; Filarszky 1907), Samland (now a part of the Kaliningrad region) (Hennings 1895), Eastern Lapland (Karsten 1882), Crimea (Léveillé 1842), Si-

${ }^{1}$ Chernigov Governorate included a large section of the modern Bryansk region 
beria (Thümen 1878, 1880a,b, 1881; Saccardo 1880), and Transbaikalia (Karsten 1906).

In 1907, Arthur Arthurovich Jaczewski published the first monograph in Russian devoted exclusively to slime molds (Jaczewski 1907). In this work, he compiled the results of previous researchers and provided a list of more than 100 species of slime molds with identification keys. Additionally, a large section of this monograph focused on various aspects of slime mold biology. This substantial publication remained the most important and, in fact, the only comprehensive source of data on myxomycetes in Russia for 86 years, until 1993.

Before the 1980s, fewer than 20 studies were published that included information on the diversity of myxomycetes. These publications addressed the myxomycete biota of the Northern European Russia (Lebedeva 1933), the former territories of Finland, that are now part of the Leningrad and Murmansk regions (Hintikka 1919), the territories of modern Nizhny Novgorod region (Murashkinskiy 1911; Javoronkowa 1914), the Kursk and the Belgorod regions (Benike 1915), Siberia (Lavrov 1927, 1929, 1931; Killermann 1943; Beglianova \& Kattsyna 1973; Petrenko 1978; Eliasson \& Lundqvist 1979), Kamchatka (Tranzschel 1914), and the southern Far East (Naoumoff 1914; Bunkina \& Koval 1967; Bunkina 1978).

The first annual myxomycete surveys in Russia took place during student summer field courses at the Zvenigorod Biological Station of the Lomonosov Moscow State University. There T.P. Sizova began research on the myxomycete species composition of the Moscow region in the 1960s. Later, in the 1990s, such studies were conducted under the guidance of T.N. Barsukova, and since 2010 V.I. Gmoshinskiy has been doing the research (Abramov et al. 2014; Adashev et al. 2014; Barsukova \& Dunayev 1997; Barsukova et al. 2012; Belyaeva et al. 2017; Gerasimovich et al. 2014; Gluhareva et al. 2017; Sizova et al. 1983; Sizova \& Titova 1985; etc.).

Starting from 1980, myxomycetes in different regions of the RSFSR (republic of the USSR, that currently comprises the most part of Russia) have been studied at the Komarov Botanical Institute of the Russian Academy of Sciences (Novozhilov 1980a,b, 1981a,b, 1984, 1985a,b, 1986a,b,c, 1987, 1988; Novozhilov \& Krussanova 1989). As a result, based on the extensive collections and the compilation of bibliographic sources, Yu.K. Novozhilov published an identification key for myxomycetes of Russia (Novozhilov 1993). This monograph remains thus far the main guide to myxomycete identification in Russia. The identification key contains information on 232 species, which only amounts to approximately $50 \%$ of the total number of species currently known in Russia. Additionally, the moist chamber culture technique (Novozhilov et al. 2000) has become a common technique of myxomycete species diversity studies. These studies and publications have stimulated interest in the field of myxomycete research in Russia. As a result, eight Ph.D. dissertations on myxomycete biodiversity were written within the last two decades.

A scientific community of researchers studying taxonomy, ecology, phylogeny, and biogeography of myxomycetes has formed in different regions of Russia. The researchers currently involved in the study of the myxomycete diversity in Russia are Yu.K. Novozhilov, O.N. Shchepin, I.S. Prikhodko, and N.A. Fedorova (St. Petersburg); M. Schnittler (Greifswald, Germany); V.I. Gmoshinskiy, A.V. Matveev, F.M. Bortnikov, N.I. Borzov, E.S. Gubanov, and A.A. Soldatenkova (Moscow); I.V. Zemlyanskaya (Volgograd); A.V. Vlasenko and V.A. Vlasenko (Novosibirsk); A.A. Shirokikh (Kirov); A.N. Lebedev (Tver); G.M. Melkumov (Voronezh); A.D. Luptakova and E.S. Korchikov (Samara); and V.N. Botyakov (Krasnodar territory). Most researchers actively collaborate and participate in joint projects throughout the country.

\section{Collections of myxomycetes in Russia}

Herbarium collections serve as one of the main tools for taxonomic, environmental, floristic, or faunistic work (Carine et al. 2018). In recent years characterized by rapid, often dramatic environmental and biodiversity changes, proper biological collections have become particularly valuable.

Traditionally, the main purpose of herbaria was to obtain data on the morphology and distribution of the deposited species, as well as to provide a retrospective view on the biodiversity of different regions. Herbaria also made it possible to restudy the 
stored collections in order to increase the objectivity and therefore the value of the previously obtained results. At present, herbarium collections have increased in importance since they are essential sources of reference specimens of morphological species. Reference specimens are used to create libraries of marker gene sequences as a precondition for DNA barcoding (Schnittler et al. 2017).

Current estimates suggest there are at least 13 myxomycete collections in Russia. Most of them are stored in various institutions, although there are a number of private collections. At the present moment, there are 25 type specimens including 16 holotypes ( 13 of them are stored in LE and 6 of them - in NSK) (see Table 1). There is a lack of information on location and condition of Trichia brevicapillata Sizova, Titova \& Darakov type specimens, that have been described in the Moscow region, and 10 taxa described by N.N. Lavrov from the vicinity of Tomsk.

In all major Russian collections myxomycete specimens are mainly stored in match boxes or occasionally in envelopes. They are situated on shelves and in herbarium cabinets in dry places without any specific conditions. In order to prevent damaging by insects some specimens undergo the procedure of preliminary freezing; in LE many specimens are isolated into individual plastic zip-lock bags to prevent the spread of insects and cross-contamination. Part of the specimens kept in the LEP herbarium have been treated with mercuric chloride.

As it was mentioned above, interest in the study of myxomycetes in Russia has grown considerably over the past 30 years, which led to the appearance of new collections in different parts of the country. The following data were obtained from a survey of collection curators. It is possible that some collections were missed during the compilation of the list.

In order to study specimens from any collection, one should contact curators of herbaria and discuss each separate inquiry.

\section{Myxomycete collection at the Mycological} Herbarium of Komarov Botanical Institute, St. Petersburg (LE)

Year of establishment: 1898. Estimated volume (specimens / different morphospecies): 30000/>300. Main regions: Belgorod, Irkutsk, Kursk, Lenin- grad, Moscow, Murmansk, Novosibirsk, Orenburg, Pskov, Sverdlovsk, Tver, Volgograd, and Voronezh regions; republics of Adygea, Crimea, Kalmykia, Karachay-Cherkessia, Karelia, and North Ossetia-Alania; Altai, Kamchatka, Krasnoyarsk, Primorye, and Trans-Baikal territory; Chukotka autonomous area. Curator of the herbarium: Olga Viktorovna Morozova(OMorozova@binran.ru), curator of the myxomycete collection: Yuri Kapitonovich Novozhilov (yurinovozhilov@gmail.com). Link and references: https://www.binran.ru/collections/, Novozhilov 1983, 2007-2020. Major contributions to the collection were made by D.A. Erastova, V.I. Gmoshinskiy, A.A. Jaczewski, A.P. Kosheleva, Yu.A. Morozova, Yu.K. Novozhilov, I.S. Prikhodko, N.A. Fedorova, M. Schnittler, O.N. Shchepin, A.V. Vlasenko, I.V. Zemlyanskaya and others.

Myxomycete collection at the Department of Mycology and Algology, Lomonosov Moscow State University (MYX)

Year of establishment: 2009. Estimated volume (specimens / different morphospecies): 16000/300. Main regions: Moscow, Samara, Tver, and Vladimir regions. Extensive collections represent Southern (Republic of Dagestan, Krasnodar territory) and Northern (Republic of Karelia, Murmansk region) Russia. Particularly valuable specimens were collected from the Russian Far East (Primorye territory). Curator: Vladimir Ivanovich Gmoshinskiy (rubisco@list.ru). Link and references: https:// micro.depo.msu.ru, Gmoshinskiy \& Matveev 2017; Gmoshinskiy et al. 2011, 2018a. Major contributions to the collection were made by M.F. Akimova, A.V. Alexandrova, E.I. Andronova, T.N. Barsukova, F.M. Bortnikov, V.N. Botyakov, N.Yu. Buchtoyarova, E.N. Vinogradskaya, V.I. Gmoshinskiy, E.S. Gubanov, N.I. Kireeva, A.D. Luptakova, A.V. Matveev, A.A. Mishulin, V.P. Prokhorov, T.P. Sizova, A.A. Soldatenkova.

Myxomycete collection of K.A. Fefelov, donated to the Museum of the Institute of Plant and Animal Ecology, the Ural Branch of the Russian Academy of Sciences

Year of establishment: 1999. Estimated volume (specimens): 8364. Main regions: Astrakhan, Che- 
Table 1. List of type specimens that are stored in Russian collections.

\begin{tabular}{|c|c|c|c|}
\hline Species & Voucher & Status & Notes \\
\hline Comatricha spinispora Novozh. \& D.W. Mitch. & LE 286575 & Holotype & \\
\hline $\begin{array}{l}\text { Cribraria bicolor S. L. Stephenson, } \\
\text { Novozh. \& P. Wellman }\end{array}$ & LE 317311 & Isotype & \\
\hline Dianema mongolicum Novozh. & LE 46251 & Holotype & $\begin{array}{l}\text { There are two intact } \\
\text { plasmodiocarps left. }\end{array}$ \\
\hline Diderma cattiense Novozh. \& D.W. Mitch. & LE 286673 & Holotype & \\
\hline Diderma dalatense Novozh., Prikhodko \& Shchepin & LE 317550 & Holotype & \\
\hline Diderma pseudotestaceum Novozh. \& D.W. Mitch. & LE 291396 & Holotype & \\
\hline Diderma velutinum Bortnikov & LE 318752 & Holotype & \\
\hline Diderma velutinum Bortnikov & MYX 8240 & Isotype & \\
\hline Diderma velutinum Bortnikov & LE 318753 & Paratype & \\
\hline Didymium reticulosporum Novozh. \& Zemly. & LE 220327 & Holotype & $\begin{array}{l}\text { There was the } \\
\text { wrong specimen } \\
\text { number given in } \\
\text { the publication } \\
\text { (Novozhilov \& } \\
\text { Zemlianskayia, } \\
\text { 2006); it should be } \\
\text { LE220327 instead } \\
\text { of LE204007. }\end{array}$ \\
\hline Didymium reticulosporum Novozh. \& Zemly. & LE 253367 & Paratype & \\
\hline Didymium reticulosporum Novozh. \& Zemly. & LE 253362 & Paratype & \\
\hline Echinostelium microsporum A. Vlasenko & NSK 1026149 & Holotype & \\
\hline Echinostelium microsporum A. Vlasenko & NSK 1026127 & Paratype & \\
\hline Echinostelium novozhilovii A. Vlasenko & NSK 1026069 & Holotype & \\
\hline Macbrideola vesiculifera Novozh. & LE 46028 & Holotype & \\
\hline $\begin{array}{l}\text { Perichaena heterospinispora Novozh., Zemly., } \\
\text { Schnittler \& S. L. Stephenson }\end{array}$ & LE 253366 & Holotype & \\
\hline $\begin{array}{l}\text { Perichaena polygonospora Novozh., Zemly., } \\
\text { Schnittler \& S. L. Stephenson }\end{array}$ & LE 253364 & Holotype & \\
\hline Perichaena taimyriensis Novozh. \& Schnittler & LE 204007 & Holotype & \\
\hline $\begin{array}{l}\text { Physarum australiense S. L. Stephenson, Novozh. } \\
\text { \& Prikhodko }\end{array}$ & LE 327851 & Holotype & \\
\hline Stemonitis pseudoflavogenita A. Vlasenko \& Novozh. & NSK 1026501 & Holotype & \\
\hline Stemonitis pseudoflavogenita A. Vlasenko \& Novozh. & LE 319201 & Isotype & \\
\hline Stemonitis pseudoflavogenita A. Vlasenko \& Novozh. & NSK 1026481 & Paratype & \\
\hline Stemonitis pseudoflavogenita A. Vlasenko \& Novozh. & NSK 1026499 & Paratype & \\
\hline Trichioides iridescens Novozh., Hooff \& Jagers & LE 266526 & Holotype & \\
\hline
\end{tabular}


lyabinsk, Kaliningrad, Kirov, Kurgan, Novosibirsk, Orenburg, Perm, Pskov, Sakhalin, Sverdlovsk, Tyumen, and Volgograd regions; Altai, Kamchatka, Khabarovsk, Krasnodar, and Primorye territories; republics of Altai, Bashkortostan, Buryatia, Crimea, Kabardino-Balkaria, Komi, North Ossetia - Alania; Chukotka autonomous area. Curator: Alexandr Anatolievich Vorobyov (eng@ipae.uran.ru). Note: K.A. Fefelov personally donated his collection to the museum in 2016 and 2017; it was designated as a separate "author's collection".

Personal collection of

I.V. Zemlyanskaya

(Volgograd)

Year of establishment: 1999. Estimated volume (specimens): 6000. Main regions: Republic of Tatarstan; Astrakhan, Moscow, Samara, Tula, and Volgograd regions. Curator: Inna Vladimirovna Zemlyanskaya (ignis@list.ru). Notes: most specimens collected by I.V. Zemlyanskaya are stored in the Myxomycetes Collection at the V.L. Komarov Botanical Institute (LE); however, some specimens, which are labeled but not identified, remain in the personal collection. Nevertheless, data on the specimens are included in the general database with unique collection numbers. After identification, the materials are transferred to the Botanical Institute of the Russian Academy of Sciences for permanent storage.

Myxomycete collection

of the Central

Siberian Botanical

Garden Herbarium (NSK)

Year of establishment: 2007. Estimated volume (specimens / different morphospecies): 4000/210. Main regions: Khanty-Mansi autonomous area; Novosibirsk, Omsk, Tomsk, and Yaroslavl regions; Altai, Buryatia, Sakha, and Tyva republics; Altai, Krasnodar, and Trans-Baikal territories. Curator: Anastasia Vladimirovna Vlasenko (anastasiamix81@mail.ru). Link: https://csbg-nsk.ru/unu_herbarium. Note: in addition to personal collections of A.V. Vlasenko and V.A. Vlasenko, it includes N.N. Lavrov's material (1916-1930) from the Tomsk Governorate.

\section{Fungarium of All-Russian Institute of Plant Protection (LEP)}

Year of establishment: 1891. Estimated volume (specimens / different morphospecies): 2000/>150. Curator: Maria Mikhailovna Gomzhina (gomzhina91@mail.ru). Reference: Berestetskaya et al. 2012. Note: the collection is currently undergoing a critical revision.

\section{Myxomycete collection of the Young Naturalists Club of Zoological Museum of Lomonosov Moscow State University}

Year of establishment: 1992. Estimated volume (specimens / different morphospecies): 1700/140. Main regions: Moscow, Tver, and Tyumen regions. Contains a significant number of specimens from Irkutsk, Murmansk, and Ryazan regions, as well as Primorye territory. Curators: Evgeny Anatolievich Dunayev (dunayeve@mail.ru), Vladimir Ivanovich Gmoshinskiy (rubisco@list.ru). References: Dunayev \& Barsukova 2002; Gmoshinskiy et al. 2018, 2020; Gmoshinskiy \& Matveev 2018. Major contributions to the collection were made by O.M. Germant, N.N. Kotelenets, E.A. Dunayev, T.N. Barsukova, V.I. Gmoshinskiy.

\section{Myxomycete collection of the Department} of Ecology, Botany and Nature Protection, Faculty of Biology, Samara University

Year of establishment: 2012. Estimated volume (specimens / different morphospecies): 700/100. Main regions: Samara and Orenburg regions. Curators: Evgeny Sergeevich Korchikov (evkor@inbox.ru) and Anna Dmitrievna Luptakova (annaloseva3545@ gmail.com).

Myxomycete collection of the herbarium of the Botanical Garden of Tver State University (TVBG)

Years of establishment: 1965-1967. Estimated volume (specimens / different morphospecies): 600/141. Main regions: Moscow, Pskov, and Tver regions. Curator: Alexandr Nikolaevich Lebedev (rumat@inbox.ru). Link: garden.tversu.ru. 
Table 2. List of federal subjects of the Russian Federation and number of the reported myxomycete species and related publications.

Federal subject

$\begin{array}{ll}\text { Code Name } & \begin{array}{l}\text { Number of } \\ \text { species }\end{array}\end{array}$

Period of study

Total

number of

publications

I journal

articles

\begin{tabular}{|c|c|c|c|c|}
\hline$A D$ & Republic of Adygeya & 2 & $2002-2005$ & $2 / 1$ \\
\hline $\mathrm{AL}$ & Republic of Altai & 165 & $1929-2020$ & $15 / 12$ \\
\hline ALT & Altai territory & 170 & $1931-2020$ & $21 / 15$ \\
\hline AMU & Amur region & 1 & 1907 & $1 / 0$ \\
\hline ARK & Arkhangelsk region & 8 & $1933-2002$ & $2 / 2$ \\
\hline AST & Astrakhan region & 104 & $1907-2020$ & $19 / 10$ \\
\hline BA & Republic of Bashkortostan & 105 & $1883-2010$ & $6 / 2$ \\
\hline BEL & Belgorod region & 5 & 1915 & $1 / 1$ \\
\hline BRY & Bryansk region & 28 & $1869-2015$ & $5 / 1$ \\
\hline BU & Republic of Buryatia & 27 & $1993-2017$ & $4 / 3$ \\
\hline CE & Chechen Republic & 3 & $1902-2005$ & $5 / 2$ \\
\hline $\mathrm{CHE}$ & Chelyabinsk region & 81 & 1907-2009 & $5 / 2$ \\
\hline $\mathrm{CHU}$ & Chukotka autonomous area & 50 & $1986-2005$ & $7 / 5$ \\
\hline CR & Sevastopol and Republic of Crimea & 145 & $1842-2020$ & $21 / 16$ \\
\hline $\mathrm{CU}$ & Chuvash Republic & 2 & 1907 & $1 / 0$ \\
\hline DA & Republic of Dagestan & 33 & $1902-2017$ & $4 / 3$ \\
\hline IN & Republic of Ingushetia & - & - & - \\
\hline IRK & Irkutsk region & 34 & $1979-2020$ & $4 / 3$ \\
\hline IVA & Ivanovo region & 1 & 2005 & $1 / 0$ \\
\hline KAM & Kamchatka territory & 41 & $1914-2017$ & $4 / 0$ \\
\hline KB & Kabardino-Balkarian Republic & 1 & 1907 & $1 / 0$ \\
\hline KC & Karachay-Cherkess Republic & 48 & $1902-2016$ & $17 / 10$ \\
\hline KDA & Krasnodar territory & 105 & $1907-2020$ & $13 / 8$ \\
\hline KEM & Kemerovo region & 1 & 1943 & $1 / 1$ \\
\hline KGD & Kaliningrad region & 6 & 1895 & $1 / 1$ \\
\hline KGN & Kurgan region & 2 & 1907-2002 & $2 / 1$ \\
\hline KHA & Khabarovsk territory & 44 & $2005-2020$ & $3 / 2$ \\
\hline KHM & Khanty-Mansi autonomous area - Yugra & 87 & $1999-2019$ & $7 / 3$ \\
\hline KIR & Kirov region & 62 & $2009-2019$ & $18 / 2$ \\
\hline KK & Republic of Khakassia & 10 & $1878-1881$ & $5 / 5$ \\
\hline KL & Republic of Kalmykia & 26 & $2003-2014$ & $7 / 4$ \\
\hline KLU & Kaluga region & 41 & $1874-2020$ & $5 / 3$ \\
\hline KO & Komi Republic & 114 & $2002-2019$ & $5 / 2$ \\
\hline KOS & Kostroma region & 9 & $1907-2005$ & $2 / 0$ \\
\hline KR & Republic of Karelia & 149 & $1882-2020$ & $17 / 13$ \\
\hline KRS & Kursk region & 59 & $1907-2014$ & $8 / 5$ \\
\hline KYA & Krasnoyarsk territory & 152 & $1878-2014$ & $29 / 16$ \\
\hline LEN & St. Petersburg and Leningrad region & 193 & $1761-2019$ & $34 / 18$ \\
\hline
\end{tabular}




\begin{tabular}{|c|c|c|c|c|}
\hline LIP & Lipetsk region & 67 & $1907-2005$ & $3 / 0$ \\
\hline MAG & Magadan region & 49 & $2000-2019$ & $3 / 1$ \\
\hline ME & Republic of Mari El & 2 & 1907 & $1 / 0$ \\
\hline MO & Republic of Mordovia & 1 & 1907 & $1 / 0$ \\
\hline MOS & Moscow and Moscow region & $252\left(229^{*}\right)$ & $1792-2020$ & $33 / 18$ \\
\hline MUR & Murmansk region & 110 & $1882-2020$ & $18 / 13$ \\
\hline NEN & Nenets autonomous area & - & - & - \\
\hline NGR & Novgorod region & 11 & $1907-2005$ & $2 / 0$ \\
\hline NIZ & Nizhny Novgorod region & 15 & $1771-2005$ & $8 / 1$ \\
\hline NVS & Novosibirsk region & 138 & $2001-2020$ & $19 / 14$ \\
\hline OMS & Omsk region & - & - & - \\
\hline ORE & Orenburg region & 50 & 1907-2009 & $5 / 1$ \\
\hline ORL & Oryol region & 14 & $2005-2019$ & $2 / 0$ \\
\hline PER & Perm territory & 74 & 2005 & $2 / 0$ \\
\hline PNZ & Penza region & 2 & $1907-2005$ & $2 / 0$ \\
\hline PRI & Primorye territory & 189 & $1914-2020$ & $12 / 10$ \\
\hline PSK & Pskov region & 78 & $1907-2020$ & $8 / 4$ \\
\hline ROS & Rostov region & 72 & $1907-2014$ & $7 / 3$ \\
\hline RYA & Ryazan region & 48 & $1907-2020$ & $5 / 2$ \\
\hline SA & Republic of Sakha (Yakutia) & 26 & $1978-2019$ & $5 / 1$ \\
\hline SAK & Sakhalin region & 14 & $2005-2015$ & $2 / 1$ \\
\hline SAM & Samara region & $44\left(43^{*}\right)$ & $1907-2019$ & $6 / 0$ \\
\hline SAR & Saratov region & 11 & $1907-2005$ & $2 / 0$ \\
\hline SE & Republic of North Ossetia - Alania & 5 & $2002-2005$ & $2 / 1$ \\
\hline SMO & Smolensk region & 77 & $1893-2005$ & $4 / 2$ \\
\hline STA & Stavropol territory & 27 & $1902-2014$ & $7 / 3$ \\
\hline SVE & Sverdlovsk region & 195 & $1907-2014$ & $25 / 7$ \\
\hline TA & Republic of Tatarstan & 45 & $1889-2005$ & $3 / 1$ \\
\hline TAM & Tambov region & 2 & 1907-2005 & $2 / 0$ \\
\hline TOM & Tomsk region & 65 & $1927-2005$ & $7 / 3$ \\
\hline TUL & Tula region & - & - & - \\
\hline TVE & Tver region & 207 & $1901-2020$ & $26 / 12$ \\
\hline TY & Republic of Tuva & 15 & 2020 & $1 / 0$ \\
\hline TYU & Tyumen region & 84 & $1907-2020$ & $4 / 2$ \\
\hline UD & Udmurtian Republic & - & - & - \\
\hline ULY & Ulyanovsk region & 5 & $1907-2005$ & $2 / 0$ \\
\hline VGG & Volgograd region & 173 & $1907-2018$ & $35 / 15$ \\
\hline VLA & Vladimir region & 40 & $1997-2020$ & $6 / 3$ \\
\hline VLG & Vologda region & 6 & $1907-2005$ & $2 / 0$ \\
\hline VOR & Voronezh region & 67 & $1907-2018$ & $12 / 5$ \\
\hline YAN & Yamal-Nenets autonomous area & 38 & $1998-2005$ & $6 / 3$ \\
\hline YAR & Yaroslavl region & 8 & $1907-2020$ & $5 / 3$ \\
\hline YEV & Jewish autonomous region & - & - & - \\
\hline ZAB & Trans-Baikal territory & 27 & $1906-2019$ & $5 / 3$ \\
\hline
\end{tabular}

* Number of species without doubtful records (marked with DR in the annotated species list). 
Myxomycete collection of the Club of Young Nature Researchers, Faculty of Biology, Lomonosov Moscow State University

Year of establishment: 2005. Estimated volume (specimens / different morphospecies): 350/50. Main regions: Kirov and Moscow regions; republics of Buryatia, Mordovia, and Tyva; Primorye territory. Curator: Anna Sergeevna Khizhnyakova (zbs_ school@mail.ru). Major contributions to the collection were made by the students of the Club of Young Nature Researchers and by A.S. Khizhnyakova.

Myxomycete collection of the Kozo-Polyansky Herbarium of Voronezh State University (VOR)

Year of establishment: 2017. Estimated volume (specimens / different morphospecies): 150/>50. Main regions: Bryansk, Lipetsk, Moscow, Oryol, Ryazan and Voronezh regions. Curator: Gavriil Mikhailovich Melkumov (agaricbim86@mail.ru). Link: http://herbarium.bio.vsu.ru.

\section{Myxomycete collection of the N.V. Rudnitsky Federal Agrarian Scientific Center of the North-East}

Year of establishment: 2016. Estimated volume (specimens / different morphospecies): 45/40. Main region: Kirov region. Curator: Alexandr Anatolievich Shirokikh (aleshirokikh@yandex.ru).

Myxomycete collection of the Herbarium of Federal Scientific Center of the East Asia Terrestrial Biodiversity, Far Eastern Branch of the Russian Academy of Sciences (VLA)

The authors reported (Bunkina \& Koval 1967) that the specimens they examined were stored in the Laboratory of Sporophytes, Biological Institute of the Far Eastern Branch, Siberian Department of the USSR Academy of Sciences (VLA). However, the fate of the collection is currently unknown.

\section{Annotated list of species}

To compile the following annotated list of myxomycetes in Russia, we used 321 literature sources published from 1761 to 2020, among them 164 journal articles, 84 conference reports, 28 book sections, 25 books, 12 dissertations, 5 student works, and 3 nature reserves annual reports. Most of these works (215) were published in Russian; 66 in English, 17 in German ( 4 of these in German and Latin), 8 in Latin, 8 in French, 4 in Ukrainian, 2 in Polish, and 1 in Hungarian. Our database contains more than 14600 records in "publication-region-species" format.

Myxomycete species names are given in accordance with Lado (2005-2020). In addition, we tend to regard Trichia ovalispora Hollós as a possible synonym of T. varia (Pers. ex J.F. Gmel.) and not as a synonym T. contorta (Ditmar) Rostaf. Pers. based on the author's drawing cited in Filarszky (1907).

The regions where a species was found (see Table 2 for the region abbreviations) and the numbers of studies that include reports of the species in this region are provided after the species name. Besides, we incorporated the cities of federal significance (Moscow, Saint Petersburg, and Sevastopol) as parts of the corresponding federal subjects: Moscow and Leningrad regions, and the Republic of Crimea, respectively.

Some older studies refer to outdated administrative units, like governorates. In such cases, we have given all modern regions that include the territories of obsolete regions as possible locations of recorded species occurrence. For example, for a species recorded from the Pskov Governorate without giving a specific location, we have cited Pskov, Novgorod, and Tver regions as the respective areas. Entries containing such controversial findings that cannot be assigned to a modern administrative unit with certainty are marked with ${ }^{\mathrm{OAU}}$ in superscript. Entries of species, that caused some doubts during primary identification, are marked with the corresponding superscripts: ${ }^{\mathrm{CF}}$, ${ }^{\mathrm{AFF}}$, ${ }^{\mathrm{AGG}}$. There are questions (Gmoshinskiy 2013, Luptakova 2019) about the accuracy of some reports considering a few species in Moscow and Samara regions. Such species records are marked with ${ }^{\mathrm{DR}}$ (dubious record). The annotated list also uses the superscripts ${ }^{1 \mathrm{IG}}$ (imprecise georeferencing) and ${ }^{\mathrm{DS}}$ (doubtful synonymy). 
Amaurochaete atra (Alb. \& Schwein.) Rostaf. BRY: 16, $101^{\mathrm{OAU}}$; CR: 148; KR: 130; LEN: 101, 182, 183, 195, 198, 212, 237, 323; MOS: 7, 66; SMO : 99, 100, 101, 198; STA: 198; SVE: 40, 41, 48, 50, 198, 201, 212; TVE: 84; VGG: 198, 220, 223, 284, 328; VLA: 173.

Amaurochaete tubulina (Alb. \& Schwein.) T.Macbr. - BU: 198; KHM: 47, 58; KIR: 108, 265; MOS: 6, 66, 198, 278; TYU: $198^{\mathrm{IG}}$.

Arcyodes incarnata (Alb. \& Schwein.) O.F.Cook AL: 134, 215; ALT: 301, 308, 317; CHE: 50, 198; CHU: 196, 198, 210, 212, 290; КНА: 84; KО: 50, 198; KYА: 120 , 122, 123, 124, 196, 198, 210, 211, 212, 290; LEN: 89, 101, 183, 195, 198, 199, 212, 237; MOS: 7, 9, 66, 70, 84, 164, 198; MUR: 196, 198, 205, 210, 212, 290; NGR: $101^{\mathrm{OAU}}$; PRI: 21; PSK: $101^{\mathrm{OAU}}$; ROS: 198, 220, 223; SVE: 50, 51, 198; TA: 101, 262; TOM: 132, 198; TVE: 19, 76, 136, 141, 198, 204; VGG: 157, 198, 212, 220, 223, 328, 336.

Arcyria affinis Rostaf. - ALT: 301, 308; AST: 198, 220, 223, 327, 328, 333; BA: 50, 198; CR: 84, 244; DA: 162; KDA: 68, 84; MOS: $1,7,11,63,64,66,70,84,164,198$; MUR: 84; PRI: 69, 74, 84, 218; PSK: 73; SVE: 49, 50, 198; TVE: 19, 76, 84, 136, 141, 198; TYU: 84; VLA: 173.

Arcyria cinerea (Bull.) Pers. - AL: 3, 134, 191, 214, 215; ALT: $215,299,301,302,306,308,310^{\mathrm{IG}}, 315,316,317$; AST: 213, 220, 221, 222, 223, 327, 328, 333; BA: 46, 50, 54; BRY: $101^{\mathrm{OAU}}$; CHE: 50, 53; CHU: 196, 198, 210, 212, 290; CR: 30, 192, 241, 242, 243, 244; IRK: 84; KDA: 68, 78, 192, 198; KHM: 47, 52, 58; KIR: 14, 108, 263, 264, 265 , 268, 272, 274, 275, 276, 291; KLU: 101; KO: 50, 270; KR: 130, 212, 254; KRS: 5, 185; KYA: 103, 116, 118, 120, 121, 122, 123, 124, 129, 196, 198, 210, 211, 212, 290; LEN: 89, 101, 161, 180, 182, 183, 195, 199, 212, 237, 323; LIP: 252; MAG: 290; MOS: $1,4,6,7,8,9,11,63,64,66,70,84,101$, 159, 160, 164, 278; MUR: 65, 84, 196, 205, 210, 212, 290; NVS: 301, 308, 310 ${ }^{\mathrm{IG}}$; ORE: 50, 53, 202; ORL: 168; PER: 50; PRI: 21, 22, 69, 74, 84, 198, 218; PSK: 73; ROS: 147, 220, 223, 335; SA: 288; SAK: 198; SAM: 153, 156; SMO: 99, 100, $101^{\mathrm{OAU}}$; STA: 101; SVE: 40, 41, 42, 44, 45, 46, 49, 50, 51, 53, 201, 212, 236; TA: 101, 262; TOM: 132; TVE: 19, 76, $84,101^{\mathrm{OAU}}, 136,141,142,165,166,179,180,182,204$; TY: 304; TYU: 84; VGG: 110, 157, 212, 220, 223, 281, 283, 284, 285, 328, 332, 336, 338; VLA: 173; VOR: 167, 170, 171, 192, 248; YAN: 50, 196, 198, 210, 212, 290.
Arcyria denudata (L.) Wettst. - AD: 198; AL: 3, 134, 191, 215; ALT: 299, 301, 302, 306, 308; AMU: 101; AST: 198, 220, 223, 327, 328, 333; BA: 50, 198; BRY: 16, 101 ${ }^{\mathrm{OAU}}, 111,198$; BU: 198; CHE: 50, 198; CR: 30, 243, 244; KAM: 251; KDA: 101, 192, 198; KHA: 198; KIR: 263, 264, 265, 268, 272, 276, 277, 291; KK: $293^{\mathrm{IG}}$; KLU: 101, 198; KOS: 101, 198; KR: 198, 212, 254; KRS: 5, 101, 185, 198; KYA: 10, 101, 120, 122, 123, 124, 196, 198, 210, 211, 212, 290, 293 ${ }^{\mathrm{IG}}$; LEN: 89, 101, 180, 182, 183, 195, 198, 199, 212, 237, 323; LIP: 198, 252; MOS: $1,4,6,7,8,9,11,18,63,66,70,84,92,93,94,101,158$, 164, 175, 198, 278; NIZ: 101, 176, 198; NVS: 301, 308; PER: 50; PRI: 21, 69, 74, 84, 198, 218; PSK: 73, 101, 140, 198; ROS: 101, 147, 335; RYA: 84, 125, 198, 321; SAM: 101, 154, 156, 198; SAR: 198; SMO: 99, 100, 101, 198; STA: 101; SVE: 42, 49, 50, 51, 198, 201, 212; TA: 101, 198, 262; TOM: 132, 198; TVE: 19, 76, 84, 101, 136, 141, 142, 180, 182, 198, 204; TYU: 84; ULY: 101, 198; VGG: 101, 110, 157, 198, 212, 220, 223, 281, 284, 328, 332, 336, 338; VLG: 101, 198; VOR: 171, 248; YAR: 101.

Arcyria ferruginea Saut. - AL: 134; ALT: 301, 307, 308; DA: 162; KO : 50, 198; KR: 130, 198, 212, 240, 254; KYA: 10; LEN: 101, 183, 195, 198, 212, 237, 247; MOS: 6, 7, 9, 66, 70, 84, 198, 278; NVS: 301, 308; PER: 50; PRI: 21, 22, 74; PSK: 140; SMO: 101, 198; SVE: 49, 50, 198, 201, 212, 236; TVE: 19, 76, 136, 198, 204.

Arcyria glauca Lister - MOS $^{\mathrm{DR}}$ : 6, 7, 198; TVE: 79, 80, 136, 282.

Arcyria globosa Schwein. - CR: 243; LEN: 89, 101, 183, 198; LIP: 198, 252; MOS $^{\mathrm{DR}}$ : 6, 7, 179, 198; NGR: 101; ORL: 168; PSK: 101; TVE: 136, 142, 179, 198, 204; VOR: 167 .

Arcyria helvetica (Meyl.) H.Neubert, Nowotny \& K.Baumann - AL: 215; ALT: 215, 307, 316; BA: 50, 54, 198; CR: 84; KHM: 52, 58; KO: 50, 198; KYA: 122, 124; MOS: 84; NVS: 301, 308; PRI: 218; SVE: 50, 198, 201, 212, 236; TVE: 19, 76.

Arcyria imperialis (G.Lister) Q.Wang \& Yu Li ALT: 215, 301, 307, 308, 316; BA: 54; IRK: 84; MOS: 84; NVS: 301, 307, 308; PRI: 84, 218.

Arcyria incarnata (Pers. ex J.F.Gmel.) Pers. - AL: 3, 
134, 214, 215; ALT: 215, 301, 308, 316; AST: 220, 223, 327, 328, 333; BA: 50, 54; BRY: $101^{\mathrm{OAU}}$, 111; CHE: 50; CHU: 196, 198, 210, 212, 290; CR: 30, 101, 192, 242, 243, 244; DA: 162; IRK: 84; KDA: 68, 78, 198; KHM: 47, 52, 58, 196; KIR: 268, 276, 277; KK: $295^{\mathrm{IG}}$; KLU: 101; KO: 50; KR: 130, 212, 240, 254; KRS: 5, 185; KYA: 10, 101, 120, 122, 123, 124, 196, 198, 210, 211, 212, 290, 295 ${ }^{\mathrm{IG}}$; LEN: 89, 96, 101, 180, 182, 183, 195, 199, 212, 237, 323; LIP: 252; MAG: 290; MOS: 1, 4, 6, 7, 8, 9, 11, $63,64,66,70,84,101,164,278$; MUR: 84 ; NIZ: $227^{\text {DS }}$, $228^{\mathrm{DS}}, 229^{\mathrm{DS}}, 230^{\mathrm{DS}}$; NVS: 301,308 ; ORE: 50; ORL: 168 ; PER: 50; PRI: 21, 74, 198, 203, 218; PSK: 73, 140; ROS: 101, 147, 220, 223; RYA: 84, 125, 321; SAM: 153, 154; SMO: $101^{\text {OAU; }}$ STA: 101 ; SVE: 40, 41, 42, 44, 45, 49, 50, 51, 201, 212, 236; TA: 101, 262; Tом: 114, 115, 132; TVE: $6,19,76,84,101^{\text {OAU }}, 135,136,139,141,142,166$, 180, 182, 204, 296; TYU: 84; VGG: 157, 212, 220, 223 , 281, 284, 285, 328, 336, 338; VOR: 248; YAN: 50, 196, 210, 212, 290 .

Arcyria insignis Kalchbr. \& Cooke - AL: 3, 214, 215; ALT: 301, 308; BA: 50; CR: 244; KDA: $78^{\text {CF }}$, 192; KO: 50; KRS: 5; KYA: 120, 122, 123, 124; LEN: 195, 212, 237; MOS: 2, 4, 6, 7, 9, 11, 64, 66, 70, 164, 179, 278; MUR: 67; NVS: 301, 308; RYA: 125, 321; SVE: 50, 201, 212, 236; TOM: $114,115,117,132$; TVE: $19,76,136$, $141^{\mathrm{CF}}, 204$; VG G: 127, 157, 212, 220, 223, 328, 337.

Arcyria magna $\mathrm{Rex}$ - KR: 198, 212, 240, 254; MOS: 66, 70; PRI: $74^{\mathrm{CF}}$; TVE: $19,141$.

Arcyria major (G.Lister) Ing - ALT: 301, 308; CR: 241; KO: 50, 198.

Arcyria marginoundulata Nann.-Bremek. \& Y.Yamam. - PRI: 218.

Arcyria minuta Buchet - AL: 215; ALT: 215, 301, $308,310^{\mathrm{IG}}, 315,316,317$; AST: $198,213,220,221,222$, 223, 328; BA: 50, 198; CR: 244; DA: 162; KDA: 90; KL: 198, 328; KLU: 8; KO: 50, 198; LEN: 182, 183, 195, 237; MOS: $1,7,9,11,63,66,70,164$; NVS: $301,308,310^{16}$; ORE: 198; PRI: 218; SAM: 153, 154; SVE: 50, 198, 236; TVE: 79, 136, 165, 166, 182, 198, 204, 282; VGG: 110 , 157, 198, 220, 223, 328, 332, 336, 338.

Arcyria obvelata (Oeder) Onsberg - AL: 3, 134, 191, 215; ALT: 215, 299, 301, 302, 306, 308, 316; AST: 198,
220, 223, 327, 328, 333; BA: 50, 198; BEL: 12; BRY: 101 ${ }^{\text {OAU }}$; CHE: 50, 198; CR: 30, 84, 144, 243, 244; DA: 57, 97, 98, 162; KAM: 251; KC: 57, 97, 98; KDA: 68; KHM: 47, 52, 58; KIR: 265, 268; KLU: $101^{\mathrm{OAU}}$; KO: 50 , 198; KR: 84, 143, 198, 212, 240, 254; KRS: 5, 185; KYA: 10, 122, 124, 196, 198, 210, 211, 212, 290; LEN: 89, 96, 101, 180, 182, 183, 195, 198, 199, 212, 237; LIP: 198, 252; MAG: 198; MOS: 1, 4, 6, 7, 8, 9, 11, 18, 63, 64, 66, 70, 84, 92, 94, 101, 164, 175, 179, 198, 278; MUR: 65, 67, 84, 96, 104, 196, 198, 205, 210, 212, 290; NIZ: 176; ORL: 168; PER: 50; PRI: 21, 22; PSK: 73, 84; ROS: 147, 198, 223, 335; RYA: 84, 125, 321; SAM: 153, 156; SMO: $101^{\text {OAU }}, 198$; SVE: $40,41,43,45,50,51,198,201,212$, 234, 236; TA: 101, 198, 262; TOM: 117, 132, 198; TVE: $19,76,84,101^{\text {OAU }}, 136,141,142,179,180,182,198$, 204; TYU: 84, 198 ${ }^{\mathrm{IG}}$; VGG: $157,198,212,220,223,284$, 328, 336, 338; VLA: 173; VOR: 101, 198, 239, 248, 249.

Arcyria occidentalis (T.Macbr.) G.Lister - AL: 215; IRK: 84; KHA: 84; MOS: 6, 66, 70, 84, 198; NVS: 301, 307, 308; SVE: 198, 236; TOM: 117, 132, 198; TVE: 19 , $76,141$.

Arcyria oerstedii Rostaf. - AL: 134, 215; ALT: 301, 307, 308; BEL: 12; CHE: 50, 198; KIR: 108, 265; KYA: 10, 198; LEN: 101, 183, 198, 247; MOS: 4, 6, 7, 9, 11, 66, 70, 84, 164, 198; MUR: 67; NVS: 301, 307, 308; PER: 50; PRI: 74; SMO: 101, 198; SVE: 51, 236; TVE: 19, 76, 136, 141, 198, 204; TYU: 84; VGG: 212, 336.

Arcyria pomiformis (Leers) Rostaf. - AL: 3, 191, 214, 215; ALT: 215, 301, 308, 316; AST: 198, 213, 220, 221, 222, 223, 327, 328, 333; B A: 50, 198; CHE: 50, 198; CHU: 196, 198, 210, 212, 290; CR: 30, 241, 243, 244; KDA: $68,192,198$; KHM: 47, 52, 58; KIR: 108, 263, 264, 265, 268, 291; KO : 50, 198; KR: 198, 212, 240, 254; KRS: 185, 198; KYA: 122, 124, 129, 196, 198, 210, 211 , 212, 290; LEN: 101, 161, 183, 195, 198, 199, 212, 237 , 247; LIP: 198, 252; MAG: 290; MOS: 1, 6, 7, 8, 9, 11, 63, 64, 66, 70, 84, 159, 160, 164, 179, 198; MUR: 196, 198, 205, 210, 212, 290; PER: 50; PRI: 69, 74, 218; PSK: 73; ROS: 198, 220, 223, 335; SAK: 198; SAM: 154, 155; SMO: 101, 198; SVE: 40, 41, 42, 44, 45, 46, 50, 51, 53, 55, 198, 201, 212, 234, 236; TA: 198; TOM: 114, 115, 117, 132, 198; TVE: 19, 84, 136, 141, 165, 166, 179, 198 , 204; TYU: 84, 198 ${ }^{\mathrm{IG}}$; VGG: 157, 198, 212, 220, 223, 281 , 284, 285, 328, 336, 338; VOR: 192, 198; ZAB: 198. 
Arcyria stipata (Schwein.) Lister - AL: 134, 215; ALT: 215, 301, 308, 316; BA: 50, 198; CR: 174, 244; KHA: 198; KHM: 52, 58; KIR: 276, 277; KYA: 112, 122, 124; LEN: 180, 181, 182, 183, 195, 198, 212, 237; MOS: 6, 7, 9, 66, 70, 84, 164, 198, 278; NVS: 301, 308; PRI: 74 , 203, 218; ROS: 147; SVE: 49, 50, 51, 198, 201, 212, 236; TOM: 114; TVE: 19, 76, 141; VGG: 198, 220, 223, 328, 329; VLA: 173.

Arcyria versicolor W.Phillips - BA: 50, 198; MOS: 6, $7^{\mathrm{CF}}, 66^{\mathrm{CF}}, 70,198,278$; NVS: 301, 307, 308; SVE: 236.

Arcyria virescens G.Lister - MOS $^{\mathrm{DR}}$ : 6, 198, 278; PRI: 218.

Badhamia affinis Rostaf. - ALT: 215, 316; AST: 198, 220, 223; IRK: 84; KIR: 276, 277; KO: 270; LEN: 182, 183, 195, 198, 212, 237; MAG: 198; MOS: 7, 11, 66, 70, 84; PRI: 74; TVE: 19, 76, 136, 179; VG G: 338.

Badhamia capsulifera (Bull.) Berk. - AST: 333; CR: 244; KGD: 91; KR: 198; KRS: 101, 185, 198; LEN: 101, $182,183,195,198,199,212,237,246,323$; MOS: $7^{\mathrm{CF}}$, 66, 70, 84, 164; PRI: 74; SMO: 101, 198; TVE: 136, 139 , 142, 166, 179, 204; VGG: 110, 198, 212, 220, 223, 284, 328,332 .

Badhamia cinerascens G.W.Martin - AL: 215; $\operatorname{MOS}^{\mathrm{DR}}: 278$.

Badhamia dubia Nann.-Bremek. - AL: 214, 215; SAM: 153.

Badhamia foliicola Lister - ALT: 301, 308, 316; AST: 198, 213, 220, 221, 222, 223, 327, 328, 333; BA: 54; CR: 192; KL: 328; KLU: 84; KR: 198, 212, 254; KYA: $120^{\mathrm{CF}}, 121,122,123^{\mathrm{CF}}, 124,198$; LEN: $182,183,195$, 198, 199, 212, 237; LIP: 198, 252; MOS: 6, 7, 8, 9, 66, 70, 84, 164, 198; PRI: 22, 74; ROS: 335; RYA: 84; TVE: 19, 136; VGG: 110, 157, 198, 212, 220, 223, 284, 285, $328,332,338$.

Badhamia goniospora Meyl. - VGG: 198, 220, 223, 284, 328, 329, 330.

Badhamia lilacina (Fr.) Rostaf. - KR: 84; KRS: 5, 198; LEN: 180; MOS: 6, 7, 66, 70, 84, 198; PSK: 73; TOM: 114, 117; TVE: 19, 136.
Badhamia macrocarpa (Ces.) Rostaf. - AL: 215; ALT: 317; AST: 198, 220, 223, 327, 328, 333; KIR: 108, 263, 264, 265, 275, 291; LEN: 182, 183, 195, 198, 212, 237; LIP: 198, 252; MOS: 6, 7, 8, 9, 11, 66, 70, 84, 164, 198; NVS: 301, 308; ORE: 50, 198; PSK: 140; ROS: 147; RYA: 125, 321; SAM: 153, 154; SE: 198; SMO: 101, 198; STA: 198; SVE: 50, 198, 201, 212; TOM: 115, 132, 198; TVE: 84, 136, 142, 166, 198, 204; VGG : 157, 198, 212, $220,223,284,328,332,338$.

Badhamia melanospora Speg. - ALT: 301, 308; Mos: 66, 70, 164.

Badhamia nitens Berk. - ALT: 317; KO: 50; MOS $^{\mathrm{DR}}$ : 6, 198, 278.

Badhamia panicea (Fr.) Rostaf. - ALT: 317; AST: 333; DA: $162^{\text {CF}}$; KHM: 52, 58; KO: 50, 198; KR: 198, 212, 254; KYA: 10, 120, 122, 123, 124, 198; LEN: 183, 195, 198, 212, 237, 286 ${ }^{\text {DS }}, 287^{\text {DS }}$; MOS: $1,6,7,9,64,66$, 70, 198; NVS: 301, 308; PSK: $73^{\text {cF }}$; ROS: 101, 198, 220 , 223; SMO: 101, 198; STA: 198; SVE: 236; TOM: 132, 198; VGG: 198, 212, 220, 223, 328, 338; VLG: 101.

Badhamia papaveracea Berk. \& Ravenel - SVE: 236.

Badhamia populina Lister \& G.Lister - KR: 198, 212, 254; VG : : 198, 220, 223, 284, 328.

Badhamia spinispora (Eliasson \& N.Lundq.) H.W.Keller \& Schokn. - ALT: 315; AST: 220, 222, 223, 328, 333; CR: 244; KL: 198, 220, 222, 223, 328; VGG: 110, 157, 198, 220, 223, 328, 332, 338.

Badhamia utricularis (Bull.) Berk. - AL: 134; AST: 198; BA: 50, 198; BRY: $101^{\text {OAU }}$, 126; CHE: 50; DA: 162; KHM: 52, 58; KIR: 268, 276, 277; KO: 50, 198; KYA: 10, 122, 124, 198; LEN: 101, 182, 183, 195, 198, 199, 212, 237, 323; MAG: 198, 290; MOS: 9, 66, 70, 84, 164, 198; NVS: 301, 308; ORE: 50, 198; PER: 50; PRI: 22, 74; PSK: 140; ROS: 147, 335; SMO: $101^{\mathrm{OAU}}$; STA: 198; SVE: 42, 50, 198, 201, 212, 236; TOM: 132, 198; TVE: 136, 198, 204; VGG : 110, 157, 198, 220, 223, 284, 328, 332, 336, 338; VOR: 248.

Badhamia versicolor Lister - KR: 198; LEN: 182, 183, 198; MOS: 66, 70. 
Badhamiopsis ainoae (Yamash.) T.E.Brooks \& H.W.Keller - AST: 329, 333; VGG: 329.

Barbeyella minutissima Meyl. - AL: 215; BA: 50, 54, 198; PER: 50; PRI: 218; SVE: 50, 51, 198, 236; TVE: $19,76,141$.

Brefeldia maxima (Fr.) Rostaf. - AL: 133, 134, 215; BRY: 16, $101^{\mathrm{OAU}}$; KIR: 277; LEN: 195, 198, 212, 237; SE: 198; SMO: 198; TOM: 133; TVE: 79, 80, 136, 204, 282; VLA: 172; VOR: 239, 248, 249.

Calomyxa metallica (Berk.) Nieuwl. - AL: 215; ALT: 215, 301, 308, 316, 317; BA: 50; CHU: 196, 198, 212, 290; CR: 244; KDA: 192, 198; КНМ: 52, 58; КО: 50, 198; KR: 198, 212, 254; KYA: 122, 124, 196, 198, 210, 211, 212, 290; LEN: 198, 199, 237; LIP: 198; MOS: 7, 66, 159, 160, 164; NVS: 301, 308; PRI: 74, 218; SA: 288; SAR: 198; SVE: 50, 51, 198, 201, 212, 236; TVE: 19, 76, 141; VOR: 198; YAN: 50, 196, 198, 210, 212, 290.

Ceratiomyxa fruticulosa (O.F.Müll.) T.Macbr. - AL: 3, 133, 134, 214, 215; ALT: 215, 301, 308, 316, 317; AST: 220, 327, 328, 333; BA: 50, 54; BRY: $101^{\mathrm{OAU}}, 126$; CHE: 50; CHU: 196, 210, 212, 290; CR: 30, 243, 244; DA: 162; IRK: 84; KAM: 251; KDA: 68; KEM: 109; KGD: 91; KHM: 47, 58; KIR: 108, 263, 264, 265, 266, 267, 268, 271, 275, 291; KLU: 8, 101; KO: 50; KOS: 101; KR: 130, 212, 254; KRS: 5, 185; KYA: 116, 129, 196, 210, 211, 212, 290; LEN: 89, 96, 101, 180, 182, 195, 199 , 212, 237; LIP: 252; MAG: 290; MOS: 1, 2, 4, 6, 7, 8, 9, 11, 63, 64, 66, 70, 84, 101, 158, 164, 175, 278; MUR: 96, 196, 205, 210, 212, 290; NIZ: 176; NVS: 301, 308; ORE: 50; ORL: 168; PER: 50; PRI: 21, 22, 74, 177, 218; PSK: 73, 84; ROS: 147, 220, 335; RYA: 125, 321; SAM: 155; SMO: 99, 100, 101 ${ }^{\mathrm{OAU}}$; SVE: 40, 41, 42, 43, 50, 51, 201, 212; TOM: 132; TVE: $6,19,76,84,101^{\mathrm{OAU}}, 136,179$, 180, 182, 204, 296; TYU: 84; VGG: 157, 212, 220, 283, 284, 328, 338; VLA: 173; VLG: 101; VOR: 239, 248, 249; YAN: 50, 196, 210, 212, 290.

Clastoderma debaryanum A.Blytt - AL: 134, 215; BU: 198; КНА: 84, 198; КНM: 52, 58; KR: 130, 198, 212, 254; KYA: 122, 124; LEN: 195, 198, 199, 212, 237; MOS: 7, 66, 70; MUR: 65; NVS: 301, 308; PER: 50; PRI: 74, 84, 203, 218; SVE: 50, 51, 198, 201, 212, 236; TVE: 19, 76, 136, 141, 142, 198, 204.
Clastoderma pachypus Nann.-Bremek. - CR: 148; PRI: 74 .

Collaria arcyrionema (Rostaf.) Nann.-Bremek. ex Lado - AL: 3, 134, 191, 215; CHE: 50, 198; KIR: 268; KO: 50, 198; KR: 198, 212, 254; KRS: 5, 198; KYA: 10, 116, 120, 122, 123, 124, 198; LEN: 89, 101, 180, 182, 183, 195, 198, 212, 237; LIP: 198, 252; MOS: 1, 6, 7, 8, 9, 11, 63, 64, 66, 70, 84, 198, 278; MUR: 65, 84; NVS: 301, 308; PER: 50; PRI: 21, 74, 203, 218; RYA: 84, 125, 321; SAK: 198; SVE: 42, 50, 198, 201, 212, 236; TOM: 114, 115, 117; TVE: 19, 76, 84, 136, 141, 180, 182, 198, 204; TYU: 84; VGG: 198, 212, 220, 223, 280, 328; VLA: 173; VOR: 248.

Collaria lurida (Lister) Nann.-Bremek. - AST: 220, 222, 223, 328; KYA: 122, 124; NVS: 301, 308; ROS: 198; SVE: 42 ${ }^{\mathrm{CF}}, 236$; VGG: 220, 223, 328.

Collaria rubens (Lister) Nann.-Bremek.-NVS: 301, 308.

Colloderma oculatum (C.Lippert) G.Lister - AL: 215; KHM: 52, 58; KR: 212, 254; LEN: 195, 197, 212, 237, 261, 282; MOS: 6, 7, 198, 278; PER: 50; PRI: 218; SVE: 51, 198; TVE: 79, 80, 136, 142, 282.

Colloderma robustum (G.Lister ex Meyl.) Meyl. KR: 198.

Comatricha alta Preuss - AL: 215; DA: 162; KO: 50, 198; MOS: 66, 70; SVE: 48, 50, 198; VGG: 88, 198, 220, 223, 281, 282, 328, 329, 338.

Comatricha anomala Rammeloo - NVS: 312.

Comatricha elegans (Racib.) G.Lister - AL: 191, 215; ALT: 301, 308; CR: 243, 244; KDA: 68; KHM: 47, 58; KO: 50, 198; KR: 198, 212, 254; KRS: 185, 198; KYA: 122, 124; LEN: 161, 182, 183, 195, 199, 212, 237; MOS: 7, 9, 11, 63, 66, 70, 198; MUR: 65, 67; ORE: 198, 202; PRI: 218; PSK: 73; ROS: 147, 198, 220, 223; SVE: 40, 41, 50, 51, 198, 201, 212, 236; TVE: 19, 76, 136, 141, 180, 181, 182, 198, 204; TYU: 198 ${ }^{\mathrm{IG}}$; VGG: 157, 198, 220, 223, 283, 284, 285, 328, 332.

Comatricha ellae Härk. - AL: 214, 215; ALT: 215, $310^{\mathrm{IG}}, 316,317$; AS T: 198, 213, 220, 223, 327, 328, 333; CHE: 50, 198; CR: 243, 244; KDA: 68, 78; KO: 50, 198; 
KR: 198; LEN: 161; MOS: 63, 66, 70, 84, 159, 160, 164; MUR: 65, 84; NVS: 301, 308, 310 ${ }^{\mathrm{IG}}$; PRI: 74; ROS: 198 , 220, 223; SVE: 236; TVE: 19, 76, 136, 141, 142, 166, 179, 204; VG : : 157, 198, 220, 223, 328.

Comatricha filamentosa Meyl. - BA: 50 $0^{\mathrm{CF}}, 198$.

Comatricha laxa Rostaf. - AL: 215; ALT: 215, 310 316, 317; AST: 198, 213, 220, 221, 222, 223, 327, 328, 333; CHE: 50, 198; CHU: 189, 196, 198, 210, 212, 290; CR: 192, 244; KDA: 68; KHM: 47, 58; KL: 198, 220, 222, 223, 328; KO: 50, 198, 270; KR: 198, 212, 254; KYA: 196, 198, 210, 211, 212, 290; LEN: 180, 182, 183, 195, 198, 212, 237; LIP: 198, 252; MOS: 4, 6, 7, 66, 70, 84, 198, 278; NVS: $301,308,310^{\mathrm{IG}}$; ORE: 50 , 198; PRI: 74, 218; ROS: 147; SAM: 154; SVE: 43, 50, 198, 201, 212, 236; TVE: $6,19,76,136,165,166,198,204$; TYU: $198^{\mathrm{IG}}$; VGG : 88, 110, 157, 198, 212, 220, 223, 281, 284 , 285, 328, 332, 338; VOR: 170; YAN: 50, 196, 198, 210 , 212, 290; ZAB: 26 .

Comatricha longipila Nann.-Bremek. - LEN: 198, 282; MOS: 66, 70, 164; SMO: 198; SVE: 44 ${ }^{\text {CF }}$ TA: 198.

Comatricha nigra (Pers. ex J.F.Gmel.) J.Schröt. - AL: 3, 191, 214, 215; ALT: 215, 301, 308, 310 317; AST: 198, 220, 222, 223, 327, 328, 333; BA: 50, 54 , 198; BRY: 101 ${ }^{\text {OAU; }}$; BU: 198; CHE: 50, 198; CHU: 189 , 196, 198, 210, 212, 290; CR: 84, 192, 243, 244; DA: 162; KAM: $251^{\text {AFF }}$; KDA: $68,78,90,192$; KHM: $47,52,58$; KIR: 275, 276; KLU: 8; KO: 50, 198; KOS: 101, 198; KR: 130, 198, 212, 254; KRS: 5, 185, 198; KYA: 120, 122, 123, 124, 196, 198, 210, 211, 212, 290; LEN: 96, 101, 180, 182, 183, 195, 198, 199, 212, 237; LIP: 198, 252; MAG: 290; MOS: $1,4,6,7,9,11,63,66,70,84,101,160$, 164, 198, 278; MUR: 65, 67, 104, 196, 198, 205, 210, 212 , 290; NVS: 301, 308, 310 $0^{\mathrm{IG}}$; ORE: 50, 198, 202; PER: 50; PRI: 22, 69, 74, 218; PSK: 73, 140; ROS: 147, 198, 220 , 223; RYA: 125, 321; SMO: 101, 198; SVE: 40, 41, 42, 43, 44, 49, 50, 51, 55, 198, 201, 212, 234, 235, 236; TA: 101, 262; TOM: 132, 198; TVE: 19, 76, 84, 135, 136, 139, 141, 142, 165, 166, 179, 182, 198, 204; TYU: 84, $198^{\mathrm{IG}}$; VGG : $88,110,157,198,212,220,223,281,284,285,328,332$, 336, 338; VLA: 173; VLG: 101; VOR: 239, 248, 249; YAN: 50, 196, 198, 210, 212, 290; ZAB: 26.

Comatricha pulchella (C.Bab.) Rostaf. - AL: 3, 215; AST: 198, 213, 220, 221, 222, 223, 327, 328, 333; BA: 50,
198; CHE: 50, 198; KHM: 47, 52, 58; KO: 50, 198; KR: 84, 198; KYA: 196, 198, 210, 211, 212, 290; LEN: 101, 180, 182, 183, 195, 198, 212, 237; MOS: 6, 7, 8, 9, 64, 66, 70, 84, 164, 198, 278; MUR: 65, 67; ORE: 50, 198; PER: 50; PRI: 218; ROS: 147, 198, 220, 223; SVE: 42, 49, 50, 51, 198, 201, 212, 236; TOM: 132, 198; TVE: 19, 84, 141; TYU: 84, 198 $8^{\mathrm{IG}}$; VGG: 110, 157, 212, 284, 328, 332, 337.

Comatricha reticulospora Ing \& P.C.Holland MOS: $84^{\mathrm{CF}}$; MUR: $84^{\mathrm{CF}}$.

Comatricha rigidireta Nann.-Bremek. - KYA: 210; SVE: $49,50,198,212^{\mathrm{CF}}$.

Comatricha suksdorfii Ellis \& Everh. - $\operatorname{MOS}^{\mathrm{DR}}$ : 6, 198, 278; SVE: 198, 236.

Comatricha tenerrima (M.A.Curtis) G.Lister - AL: 3, 215; ALT: 317; BA: 50, 198; CHE: 50; CR: 148; KHM: 47, 58; KRS: 185, 198; KYA: 121, 122, 124; LEN: 182, 183, 195, 198, 212, 237; Mos: 1, 4, 6, 7, 66, 70, 198; NVS: 301, 308; PER: 50; PRI: 74; SVE: 50, 198, 201, 212; TVE: 19, 76, 136, 198, 204; TYU: $198^{\mathrm{IG}}$.

Craterium aureum (Schumach.) Rostaf. - ALT: 301, 308; KDA: 90; KR: $130^{\mathrm{CF}}$, 198; KYA: 122, 124; LEN: 101, 198; MOS: 6, 7, 11, 66, 70, 84, 198; SVE: 49, 50, 198, 201, 212, 236; VGG: 157, 198, 212, 220, 223, 328.

Craterium concinnum Rex - Mos: 6, 7, 66, 70, 198; PRI: 218.

Craterium leucocephalum (Pers. ex J.F.Gmel.) Ditmar-AL: 191, 215; ALT: 299, 301, 308; AST: 198, 220 , 222, 223, 327, 328, 333; BA: 54; CR: 30, 242, 243, 244; KDA: 90; KHA: 198; KOS: 101, 198; KR: 198, 212, 254; KRS: 5, 185, 198; KYA: 122, 124, 196, 198, 210, 211 , 212, 290; LEN: 101, 182, 183, 195, 198, 199, 212, 237, 323; LIP: 198, 252; MAG: 288, 290; MOS: 1, 6, 7, 11, 63, 66, 70, 84, 198; MUR: 84 ; NGR: $101^{\text {OAU; }}$ NVS: 301,308 ; ORE: 50, 198; PRI: 74, 203; PSK: 101 ${ }^{\text {OAU }}$, 198; ROS: 147, 335; SAM: 153, 154; SVE: 45, 49, 50, 198, 201, 212 , 236; TOM: 132, 198; TVE: 19, 76, 136, 204; VGG: 157 , $198,212,220,223,281,284,285,328,336,338$; YAN: 50, 196, 198, 210, 212, 290; YAR: 101, 198.

Craterium minutum (Leers) Fr. - AL: 3, 134, 215, 300; ALT: 215, 316; CR: 242, 243, 244; KO: 50, 198; 
KRS: 185, 198; LEN: 101, 180, 182, 183, 195, 198, 199, 212, 237, 323; LIP: 198, 252; MOS: 6, 7, 8, 11, 63, 64, 66, 70, 84, 198; MUR: 96, 104; NVS: 301, 308; PRI: 22 , 218; SMO: 99, 100; SVE: 49, 50, 198, 201; TVE: 19, 76, 79, 80, 136, 180, 182, 204, 282, 296; TYU: 84; VGG: 198, 220, 223, 328, 338; VLA: 173; YAR: 101, 198.

Craterium obovatum Peck - AL: 215; IRK: 84; KHA: 198; LEN: 182, 183, 195, 198, 212, 237; MOS: 7, 8, 66; PRI: 74, 203, 218; TVE: 136, 204.

Cribraria argillacea (Pers. ex J.F.Gmel.) Pers. - AL: 3, 134, 191, 215; ALT: 194, 301, 308; ARK: 143; BA: 50, 54, 198; CR: 243, 244; IRK: 84; KAM: 251; KHM: 52, 58; KLU: 101, 198; KO: 50, 198; KR: 198, 212, 254; KRS: 185, 198; KYA: 10, 122, 124, 194, 198; LEN: 89, 101, 180, 182, 183, 195, 198, 199, 212, 237, 323; MOS: $1,4,6,7,9,11,63,66,70,84,101,198,278$; MUR: 65 ; PER: 50; PRI: 21, 22, 194, 218; PSK: 140; RYA: 84; SAK: 198; SAM: 155, 156; SMO: 101, 198; STA: 198; SVE: 49, 50, 51, 198, 201, 212, 236; TA: 198; TVE: 19, 76, 136, 142, 179; TYU: 84; VGG: 198, 212, 220, 223, 328, 338; VLA: 173; VOR: 171, 248.

Cribraria atrofusca G.W.Martin \& Lovejoy - KYA: $196^{\mathrm{CF}}, 198,211^{\mathrm{CF}}, 212^{\mathrm{CF}}, 290^{\mathrm{CF}}$; MOS: 63, 66, 70; NVS: 26; SVE: 198, 236.

Cribraria aurantiaca Schrad. - AL: 3, 134, 191, 215; ALT: 194, 215, 301, 308, 316; BA: 50, 198; CR: 30, 243, 244; KHM: 47, 58; KO: 50, 198; KR: 198, 212, 254; KRS: 185, 198; KYA: 10, 122, 124, 129, 198, 212; LEN: 89, 101, 182, 183, 195, 198, 199, 212, 237, 323; LIP: 198, 252; MOS: $1,4,6,7,11,63,66,70,84,164^{\mathrm{CF}}, 198$; MUR: 196, 198, 205, 210, 212, 290; PRI: 21, 22, 194, 218; ROS: 147, 335; RYA: 125, 321; SAK: 198; SMO: 101, 198; STA: 198; SVE: 40, 41, 50, 51, 198, 201, 212, 236; TA: 101, 198, 262; TOM: 132, 198; TVE: 19, 76, 136, 141, 142, 198, 204; TYU: 84, 198 ${ }^{\mathrm{IG}}$; VGG: 157, 198, 212, 220, 223, 328, 338.

Cribraria cancellata (Batsch) Nann.-Bremek. - AL: 3, 134, 191, 215; ALT: 194, 215, 299, 301, 302, 306, 308, 316; BA: 50, 54, 198; CHE: 50, 198; CR: 30, 241, 243, 244; IRK: 84; KAM: 251; KDA: 68; KHA: 198; КHM: 47, 52, 58; KLU: 101, 198; KO: 50, 198; KR: 143, 198, 212, 254; KRS: 5; KYA: 10, 122, 124, 129, 194, 198; LEN: 89, 96, 101, 180, 182, 183, 195, 198, 199, 212, 237, 323; LIP: 198, 252; MOS: 1, 4, 6, 7, 8, 9, 11, 63,
64, 66, 70, 84, 101, 164, 179, 278; MUR: 65, 67, 196, 205, 210, 212, 290; NIZ: 101, 198; NVS: 301, 308; ORE: 50, 198; PER: 50; PRI: 21, 69, 74, 84, 194, 203, 218; PSK: 73; ROS: 147; RYA: 84, 125, 321; SAM: 153, 154; SMO: 101, 198; SVE: 40, 41, 49, 50, 51, 198, 201, 212, 236; TA: 101, 198, 262; TOM: 132, 198; TVE: 19, 76, 84, 136, 139, 141, 142, 166, 179, 180, 182, 198, 204; TYU: 84, 198 ${ }^{\mathrm{IG}}$; VGG: $198,212,220,223,281,328,336,338$; VLA: 173; VOR: 167, 170.

Cribraria confusa Nann.-Bremek. \& Y.Yamam. KO: 50, 198.

Cribraria costata Dhillon \& Nann.-Bremek. KO: 50, 198.

Cribraria elegans Berk. \& M.A.Curtis - AL: 191, 300; TVE: 19.

Cribraria exigua Meyl. - KO: 50, 198; TYU: $198^{\mathrm{IG}}$.

Cribraria filiformis Nowotny \& H.Neubert KR: 198.

Cribraria intricata Schrad. - AL: 3, 215; ALT: 215, 301, 308; KHM: 52, 58; KIR: 108, 265; KYA: 122, 124; MOS: 1, 4, 6, 7, 8, 9, 63, 66, 70, 198; NVS: 301, 308; PRI: 218; SVE: 50, 198, 201, 212, 236; TVE: 19, 136, 204; TYU: 84; VGG: 27 .

Cribraria languescens Rex - AL: 215; ALT: 215, 301, 308, 316; MOS: 1, 6, $7^{\mathrm{CF}}$, 66, 70, 84, 198; PRI: 218; SVE: 50, 198, 201, 212; TVE: 19, 76, 141; VGG: 198, 212, $220,223,328,330$.

Cribraria lepida Meyl. - ALT: 303, 317; NVS: 303; VGG: 27 .

Cribraria macrocarpa Schrad. - AL: 215; KDA: 68; LEN: 89, $101^{\mathrm{OAU}}, 183,195,198,212,237$; MOS: 7, 66, 70, 84; NGR: $101^{\mathrm{OAU}}$; PSK: $101^{\mathrm{OAU}}$; SVE: 50, 198, 201, 212, 236; TYU: 84.

Cribraria macrostipitata H.Neubert \& Nann.Bremek. - AL: 198, 215.

Cribraria microcarpa (Schrad.) Pers. - AL: 198, 215; ALT: 194, 215, 301, 308; BA: 50, 198; CR: 244; 
KIR: 267, 268, 275; KR: 198, 212, 254; KYA: 122, 124, 196, 198, 210, 211, 212, 290; LEN: 323; MOS: 1, 4, 6, 7, 64, 66, 70, 84, 164, 198; PRI: 69, 74, 218; PSK: 73; ROS: 147; RYA: 84; SVE: 236; TA: 101, 198, 262; TVE: 19, 76, 84; TYU: 84.

Cribraria minutissima Schwein. - AL: 3, 191, 215, 300; ALT: 194, 301, 308; BU: 194, 198; CHE: 50, 198; CR: 243, 244; KHA: 198; KR: 198, 212, 254; MOS: 7, 11 , 66, 70; MUR: 65 ${ }^{\mathrm{CF}}$, 67; PRI: 69, 74, 194, 203, 218; TVE: 19, 76, 141; ZAB: 194.

Cribraria mirabilis (Rostaf.) Massee - AL: 198; KO: 50; SVE: 236.

Cribraria oregana H.C.Gilbert - CHE: 50; KO: 50, 198; KR: 198; MOS: 84; PRI: 84, 218; TYU: 84.

Cribraria piriformis Schrad. - AL: 215; CHE: 50, 198; KR: 198; LEN: 89, 101, 182, 183, 195, 198, 212, 237; MOS: 6, 7, 66, 70, 84, 198; NGR: 101; PRI: 21, 194; PSK: 101; SVE: 50, 198, 201, 212, 236; TVE: 19, 84, 136, 142, 180, 182, 198.

Cribraria purpurea Schrad. - AL: 133, 134, 215; ALT: 194, 301, 308; KHM: 52, 58; KO: 50, 198; KR: 198, 212, 254; KYA: 122, 124; LEN: 101, 180, 182, 183, 195, 197, 198, 212, 237, 282, 323; MOS: 6, 84, 198; MUR: 65; NVS: 301, 308; PRI: 198; PSK: 140; SMO: 101, 198; SVE: 50, 51, 198, 212, 236; TVE: 19, 76, 136, 141, 142.

Cribraria rubiginosa Fr. - AL: 191, 198, 215; MOS $^{\mathrm{DR}}$ : $6,198$.

Cribraria rufa (Roth) Rostaf. - AL: 3, 215; ALT: 215, 301, 308, 316; BA: 50, 198; CHE: 50; CR: 244; KHM: 47, 52, 58; KO: 50, 198; KR: 130, 198, 212, 254; KRS: 5, 185, 198; KYA: 194; LEN: 89, 180, 182, 183, 195, 198, 212, 237, 323; LIP: 198, 252; MOS: 1, 6, 7, 11, 66, 70, 84, 198; NVS: 301, 308; ORE: 50; PER: 50; PRI: 21, 194, 218; PSK: 140; RYA: 125, 321; SVE: 40, 41, 45, 50, 198, 201, 212, 236; TVE: 19, 76, 84, 136, 141, 179; TYU: 198 ${ }^{\mathrm{IG}}$; VGG: 157, 198, 220, 223, 328, 330; VLA: 173.

Cribraria splendens (Schrad.) Pers. - AL: 134, 215; KHA: 198; КНM: 52, 58; KO: 50, 198; KR: 198, 212, 254; LEN: 101, 183, 195, 198, 212, 237, 323; MOS $^{\mathrm{DR}}$ : 6, 101, 198; SVE: 50, 198, 201, 212, 236; TA: 198; TVE: 136, 142.
Cribraria tenella Schrad. - AL: 215; ALT: 215, 316; BA: 50, 54, 198; BU: 198; CHE: 50, 198; CR: 244; KDA: 192, 194, 198; KHМ: 47, 52, 58; KO: 50, 198; KYA: 122, 124; LEN: 101, 183, 194, 195, 198, 212, 237, 323; MOS: 1, 7, 66, 70, 84; PRI: 69, 74, 218; PSK: 73; RYA: 84, 125, 321; SVE: 50, 51, 198, 201, 212, 236; TA: 101, 198, 262; TVE: 19, 76, 141; TYU: 198 ${ }^{\mathrm{IG}}$; VGG : 198, 212, 220, 223, 328,330 .

Cribraria violacea Rex - AL: 215; ALT: 215, 301, 308, 315, 317; AST: 198, 213, 220, 222, 223, 327, 328, 333; BA: 50, 198; CR: 241, 242, 243, 244; KDA: 78, 192 , 194, 198; KHM: 26; KIR: 108, 265; KLU: 8; KYA: 120, 121, 122, 123, 124, 196, 198, 210, 211, 212, 290; LEN: 199; MOS: 7, 8, 66, 84, 159, 164; NVS: 301, 308; PER: 50; PRI: 74, 218; ROS: 147; SA: 288; SAM: 155; SVE: 50, 51, 198, 201, 212, 236; TVE: 165, 166; VGG: 157, 198, 212, 220, 223, 328, 338.

Cribraria vulgaris Schrad. - AL: 191, 215; ALT: 194, 215; CHE: 198; CR: 243, 244; KDA: 90; KO: 50, 198; KR: 198, 212, 254; KYA: 120, 122, 123, 124, 196, 198, 210, 211, 212, 290; LEN: 183, 194, 195, 198, 199, 237, 323; MOS: $1,6,7,11,63,66,70,84,158,198$; MUR: 67, 84; PRI: 194; SMO: 99, 100; SVE: 50, 198, 201, 212, 236; TA: 262; TVE: 19, 76, 136, 141, 142; TYU: 84.

Diachea bulbillosa (Berk. \& Broome) Lister - PRI: 218.

Diachea leucopodia (Bull.) Rostaf. - CR: 30, 242, 243, 244; KHA: 198; LEN: 101, 180, 182, 183, 195, 198, 212, 237, 323; MOS: 4, 6, 7, 66, 70, 84, 198; NVS: 27; PRI: 22, 74, 203, 218; ROS: 335; SAK: 198; SMO: 101, 198; TVE: 19, 76; VGG: 157, 198, 212, 220, 223, 328; VOR: 167, 239, 249.

Diachea splendens Peck - KHA: 198; LEN: 195, 197, 212, 237, 282; PRI: 74, 203; SAK: 198.

Diachea subsessilis Peck - PRI: 21; VGG: 198, 220, 223, 284, 285, 328, 329, 330.

Diacheopsis effusa Kowalski - KR: 198; MUR: 34, 36, 196, 205, 210, 212, 290.

Diacheopsis metallica Meyl. - BU: 198; KHM: 52; KYA: 120, 122, 123, 124, 198; MUR: 34, 36; PER: 50; SVE: 44 ${ }^{\mathrm{CF}}, 48,50,198,236$; TVE: 20. 
Diacheopsis nannengae G.Moreno, Illana \& Heykoop - MUR: $65^{\mathrm{CF},} 67$.

Diacheopsis reticulospora Mar.Mey. \& Poulain MUR: 34, 36.

Dianema corticatum Lister - AL: 215; KL: 198, 220, 223, 328; KR: 198, 212, 254; PRI: 218; ROS: 220, 223; TOM: 117, 132, 198; VGG: 212, 328.

Dianema depressum (Lister) Lister - CR: 244; VOR: 239, 249.

Dianema harveyi Rex - ALT: 317; CR: 148.

Dianema mongolicum Novozh. - AL: 214, 215.

Dianema subretisporum Kowalski - KC: 34, 217, 255.

Dictydiaethalium plumbeum (Schumach.) Rostaf. - AL: 215; CHU: 196, 198, 210, 212, 290; CR: 148, 243 , 244; KRS: 185, 198; KYA: 122, 124; LEN: 195, 198, 199 , 212, 237; MAG: 198; MOS: 7, 9, 11, 66, 70, 84, 164, 198; PSK: 73, 140; STA: 198; SVE: 50; TVE: 136, 142, 165, 166, 198, 204; YAN: 50, 196, 198, 210, 212, 290.

Diderma alpinum (Meyl.) Meyl. - KAM: 219; KC: 34, 217; KR: 35; LEN: 34, 35, 261; MOS: 6, 7, 66, 70, 198; MUR: 34, 36, 67.

Diderma asteroides (Lister \& G.Lister) G.Lister KO: 50, 198; KR: 198, 212, 254.

Diderma brooksii Kowalski - PER: 50; SVE: 198.

Diderma chondrioderma (de Bary \& Rostaf.) Kuntze - KDA: 68; MOS: 66, 70, 164; PRI: 218; SA: 288; TVE: 166.

Diderma cinereum Morgan - AST: 198, 213; MOS: 63, 84; PSK: 73; TVE: 19, 76.

Diderma cor-rubrum T.Macbr. - PRI: 218.

Diderma crustaceum Peck - MOS ${ }^{\mathrm{DR}}$ : 15; SAM: 153.
Diderma deplanatum Fr. - ALT: 315; AST: 220, 222, 223, 328; KYA: 120, 122, 123, 124, 198; LEN: 182, 183, 195, 198, 212, 237, 323; MOS: 66; MUR: 196, 198, 205, 210, 212, 290; NVS: 301, 308; PRI: 218; ROS: 335; TVE: 19, 76; ZAB: 26.

Diderma effusum (Schwein.) Morgan - ALT: 301, 308, 310 ${ }^{\mathrm{IG}}$, 316; CR: 174; KDA: 90; KR: 198; KYA: 122, 124; MOS: 6, 7, 66, 70, 84, 198, 278; NVS: 301, 308, 310 ${ }^{\mathrm{IG}}$; PRI: 74, 84, 218; SA: 288; TVE: 76; VOR: 171, 248, 249.

Diderma europaeum (Buyck) Kuhnt - КАМ: 219; KC: 34, 217; KR: 34; LEN: 261; MUR: 34, 36.

Diderma evelinae (Meyl.) Kowalski - ALT: 301, 307, 308.

Diderma fallax (Rostaf.) E.Sheld. - KAM: 297; KC: 34, 217; KHM: 52, 58; MOS $^{\mathrm{DR}}$ : 6, 198; PER: 50; TVE: 79, 80, 136, 179, 198, 204, 282.

Diderma floriforme (Bull.) Pers. - CHE: 50, 198; KO: 50, 198; KRS: 185, 198; LEN: 182, 183, 195, 197, 198, 212, 237, 282; MOS: 7, 66, 70; PRI: $218^{\mathrm{CF}}$; SAK: 198; SVE: 50, 198, 236; TVE: 19, 141.

Diderma globosum Pers. - ALT: 317; CR: 242, 243, 244; KLU: 101, 198; KR: 198, 212, 254; LEN: 101, 182, 183, 195, 198, 212, 246, 323; MOS: 6, 7, 66, 198; PRI: 218; ROS: 335; SAK: 198; SAM: 153, 154; SVE: 50 ${ }^{\mathrm{CF}}$; TA: 101, 198, 262; TVE: 182; VGG: 198, 220, 223, 284, $285,328$.

Diderma hemisphaericum (Bull.) Hornem. - AL: 3, 215; ALT: 317; BA: 253; LEN: 101, 182, 183, 195, 198, 212, 237, 246, 323; Mos: 8, 9, 66, 70, 164, 198; NVS: 301, 307, 308; ORE: 101, 198; TVE: 136, 165, 166, 198, 204; VGG: 157.

Diderma meyerae H.Singer, G.Moreno, Illana \& A.Sánchez - KC: 34, 217, 255; MUR: 34, 36, 67.

Diderma montanum (Meyl.) Meyl. - BA: 54; CR: 174; KDA: 192, 198; KHA: 198; KO: 50, 198; LEN: 180, 182, 183, 195, 198, 212, 237; MOS: 64, 164, 198; NVS: 26; ORE: 198, 202; PER: 50; PRI: 74, 203; SA: 288; SVE: 50, 51; TVE: 19, 76, 136. 
Diderma niveum (Rostaf.) E.Sheld. - ARK: 143; CHU: 196, 198, 210, 212, 290; CR: 29, 30; KAM: 198, 219; KC: 34, 217, 258; KR: 34, 35; LEN: 34, 35, 101, $183,188,195,197,198,212,237,258,261,282 ;$ MOS $^{\text {DR: }}$ 6, 101, 198; MUR: 34, 36, 196, 198, 205, 210, 212, 290; SMO: 101, 198; SVE: 51; TVE: 19, 20, 136, 180.

Diderma ochraceum Hoffm. - LEN: 101; MOS: 6, 101; PER: 50; SMO: 101; TA: 101, 198, 262.

Diderma pseudotestaceum Novozh. \& D.W.Mitch. - PRI: 218.

Diderma radiatum (L.) Morgan - ALT: 215, 301, 308; CHE: 50, 198; CHU: 196, 198, 210, 212, 290; CR: 242, 243, 244; IRK: 84; KHA: 198; KHM: 47, 52, 58; KO: 50, 198; KR: 96, 198, 212, 254; KYA: 122, 124, 196, 198, 210, 211, 212, 290; LEN: 101, 182, 183, 195, 198, 212, 237, 246, 323; MOS: 1, 6, 7, 64, 66, 70, 84, 93, 101, 175, 198, 278; MUR: 65, 67, 84; PER: 50; PRI: 74, 203; PSK: 73; SMO: 101, 198; STA: 198; SVE: 49, 50, 198, 201, 212, 236; TA: 198; TVE: 19, 76, 84, 141; TYU: 84 , $198^{\mathrm{IG}}$; VOR: 248.

Diderma saundersii (Berk. \& Broome ex Massee) E.Sheld. - MOS: 66; PRI: 74; ZAB: 26.

Diderma sauteri (Rostaf.) E.Sheld. - LEN: 89, 101, 195, 198, 212, 237; PRI: 218.

Diderma simplex (J.Schröt.) E.Sheld. - KYA: 120, 122, 123, 124, 198; MOS $^{\mathrm{DR}}$ : 6, 7, 15, 198, 278; SVE: 50, 198, $201^{\mathrm{CF}}, 212^{\mathrm{CF}}$; TVE: $136,142^{\mathrm{CF}}$.

Diderma spumarioides (Fr. \& Palmquist) Fr. - CR: 30, 174, 242, 243, 244; KAM: 198; KDA: 90, 192, 198; KHA: 198; LEN: 182, 183, 195, 198, 212, 237, 246; LIP: 198, 252; MOS: 7, 66, 70, 84; NGR: $101^{\text {OAU }}$; PRI: 22, 84 , 218; PSK: 101 ${ }^{\text {OAU }}$, 198; ROS: 147; SMO: 101, 198; SVE: 49; TA: 101, 198, 262; TVE: 19, 76, 136, 142; VGG: 198.

Diderma testaceum (Schrad.) Pers. - AL: 215; CR: 174, 242, 243, 244; KR: 130; LEN: 101, 182, 195, 198, 237, 323; MOS: 66, 70; PRI: 218; SMO: 101, 198; TVE: 19, 76.

Diderma umbilicatum Pers. - ALT: 215; BA: 54; MOS: 198; PER: 50; TVE: 19.
Diderma velutinum Bortnikov - PRI: 17.

Didymium anellus Morgan - AL: 214, 215; ALT: 315, 317; AST: 198, 213, 220, 222, 223, 327, 328, 333; CHE: 50, 53, 198; CR: 174, 242, 243, 244; KL: 220, 222 , 223, 328; KYA: $120^{\mathrm{CF}}, 121,122,123,124,198$; LEN: 182, 183, 195, 198, 199, $212^{\mathrm{AGG}}$, 237; MOS: 66, 84, 164; NVS: 301, 308; ORE: 198, 202; PRI: 74, 218; SA: 288; SAR: 198; TVE: 76, 136, 165; VGG: 110, 157, 198, 220, 223, 284, 328, 332, 338.

Didymium annulisporum H.W.Keller \& Schokn. NVS: 301, 307, 308.

Didymium bahiense Gottsb. - AST: 213, 220, 223, 328, 333; PRI: 218.

Didymium clavus (Alb. \& Schwein.) Rabenh. - AL: 215; ALT: 315; AST: 198, 220, 223, 327, 328, 333; BA: 50, 54, 198; CHU: 196, 198, 210, 212, 290; CR: 144, 174, 242, 243, 244; KHM: 52, 58; KIR: 263, 265; KO: 50, 198; KOS: 101, 198; KR: 198; KRS: 185, 198; KYA: 10, 120, 121, 122, 123, 124, 198; LEN: 101, 180, 182, 183, 195, 198, 199, 212, 237, 323; LIP: 198, 252; MAG: 290; MOS: 4, 6, 7, 9, 11, 63, 66, 70, 84, 164, 198; NVS: 301, 308; PER: 50; PRI: 21, 218; ROS: 147; SMO: 101, 198; SVE: 50, 51, 198, 201, 212, 236; TVE: 19, 76, 136, 182; VGG: 198, 212, 220, 223, 284, 285, 328, 338; YAN: 50, 196, 198, 210, 212, 290.

Didymium comatum (Lister) Nann.-Bremek. ALT: 301, 308; LEN: 323; NVS: 301, 308; ORE: 53.

Didymium crustaceum Fr. - ALT: 315; AST: 198, 220, 223, 327, 328, 333; BRY: $101^{\mathrm{OAU}}$; CHU: 196, 198, 210, 212, 290; KHM: 47, 58; KLU: 101 ${ }^{\mathrm{OAU}}$; LEN: 101, 183, 195, 198, 212, 237, 246; Mos: 6, 7, 8, 15, 66, 70, 84, $101^{\mathrm{OAU}}, 164,198$, 238; ROS: 147; SMO: $101^{\mathrm{OAU}}, 198$; SVE: 51; TOM: 132, 198; TVE: 6, 101 ${ }^{\text {OAU }}, 136,204$; TYU: 198 ${ }^{\mathrm{IG}}$; VGG: 157, 198, 212, 220, 223, 284, 328.

Didymium dachnayum (G. Walker, Silberman, Karpov, Preisfeld, P. Foster, A.O. Frolov, Novozh. \& Sogin) Fiore-Donno, Kamono \& Caval.-Sm. - LEN: $59,322$.

Didymium difforme (Pers.) Gray - AL: 214, 215; ALT: 315, 316, 317; AST: 198, 213, 220, 221, 222, 223, 
327, 328, 333; BA: 46, 50, 198; CHE: 50, 53, 198; CHU: 196, 198, 210, 212, 290; CR: 174; KDA: 78; KL: 220, 222, 223, 328; KLU: 101, 198; KOS: 101; KR: 198, 212 , 254; KYA: $120,121,122,123,124,196,198,210,211$, 212, 290; LEN: 101, 161, 182, 183, 195, 198, 199, 212 , 237, 323; LIP: $101^{\mathrm{OAU}}$, 198, 252; MO: $101^{\mathrm{OAU}}$; MOS: 6, 7 , 8, 9, 15, 66, 70, 84, 101, 164, 198, 278; MUR: 196, 198, 205, 210, 212, 290; NIZ: 101 ${ }^{\mathrm{OAU}}, 102,176$; ORE: 53, 198, 202; PNZ: 101 ${ }^{\mathrm{OAU}}$; PRI: 218; PSK: 101, 198; RYA: 101 ${ }^{\mathrm{OAU}}$; SAR: 198; SMO: 101, 198; SVE: 46, 50, 51, 53, 198, 201, 212; TA: 101, 198, 262; TAM: $101^{\mathrm{OAU}}, 198$; TVE: 136, 142, 165, 166; TY: 304; VGG: 101, 110, 157, 198, 220, 223, 284, 328, 332, 336, 338; VOR: $101^{\mathrm{OAU}}$, 248, 249; YAN: 196, 198, 210, 290.

Didymium dubium Rostaf. - ALT: 301, 308, 315, 317; AST: 198, 220, 222, 223, 327, 328, 333; BU: 198; IRK: 198; KAM: 219; KC: 34, 217, 255; KR: 198, 212 , 254; KYA: 196, 198, 210, 211, 212, 290; LEN: 182, 183, 195, 198, 199, 212, 237; MOS: 7, 8, 9, 66, 70, 198; MUR: 34, 36, 196, 198, 205, 210, 212, 290; NVS: 301, 308; ORE: 198, 202; RYA: 125, 321; SA: 288; SVE: 50, 198, 236; VGG : 157, 198, 212, 220, 223, 284, 328, 332, 338; YAN: 196, 198, 210, 290; ZAB: 26.

Didymium flexuosum Yamash. - MOS: $66^{\mathrm{CF}}$; TVE: 166 ${ }^{\mathrm{CF}}$; VGG: 198, 220, 223, 257, 328, 329.

Didymium inconspicuum Nann.-Bremek. \& D.W.Mitch. - AST: 220, 222, 223, 328, 333.

Didymium iridis (Ditmar) Fr. - AL: 215; ALT: 301, 308, 317; AST: 198, 213, 220, 222, 223, 328, 333; ВA: 46, 50, 198; CHE: 50, 53, 198; KDA: 78; KRS: 5, 198; MOS: 7, 8, 63, 66, 70, 84; NVS: 301, 308; ORE: 53; SVE: 46, 50, 53, 198, 201, 212; TVE: 79, 80, 136, 137, 139, 166, 282; VGG: 110, 157, 198, 220, 223, 284, 328, 332; VOR: 167, 170, 192, 198.

Didymium karstensii Nann.-Bremek. - ORE: 198, 202; PRI: 218.

Didymium leoninum Berk. \& Broome - PRI: 218 .

Didymium listeri Massee - KHA: 198; KR: 198; LEN: 198.

Didymium megalosporum Berk. \& M.A.Curtis - Mos: 84; PRI: 218; VGG: 198, 220, 223, 281, 282, 328, 338.
Didymium melanospermum (Pers.) T.Macbr. ALT: 301, 308; AST: 198, 220, 223, 327, 328, 333; BRY: 101 ${ }^{\mathrm{OAU}}$; BU: 198; CE: 57, 97, 98, 101; CHU: 196, 198, 210, 212, 290; CR: 30, 242, 244; KDA: 90; КнM: 47, 58; KLU: 101, 198; KO: 50, 198; KR: 198, 212, 254; KRS: 185 , 198; KYA: $10,120,122,123,124,196,198$, 210, 211, 212, 290; LEN: 96, 101, 180, 182, 183, 195, 198, 199, 212, 237, 261, 323; LIP: 198, 252; MAG : 290; MOS: 4, 6, 7, 8, 11, 15, 63, 64, 66, 70, 84, 101, 198, 278; MUR: 65, 67; NIZ: 176; PER: 198; PNZ: 198; PRI: 21, 218; RYA: 84; SA: 288; SAK: 198; SAM: 198; SMO: 100, $101^{\mathrm{OAU}}$, 198; SVE: 49 ${ }^{\mathrm{CF}}, 50,51,101,198,201,212$, 236; TOM: 132, 198, 233; TVE: $19,76,84,101^{\mathrm{OAU}}, 135$, 136, 139, 141, 165, 166, 180, 182, 198, 204; TYU: $198^{\mathrm{IG}}$; VGG: $110,198,212,220,223,284,285,328,332$; VLA: 6, 173, 198; YAN: 50, 196, 198, 210, 212, 290; YAR: 84.

Didymium mexicanum G.Moreno, Lizárraga \& Illana - AST: 198, 220, 222, 223, 328, 329, 333; KL: 328; VGG: 110, 332.

Didymium minus (Lister) Morgan - ALT: 301, 308; BRY: 111; CHE: 50, 198; CR: 174; KDA: 90; KHM: 47, 58; Kо: 50, 198; MOS: 6, 7, 8, 9, 11, 63, 66, 70, 84, 198, 238; PER: 50; PRI: 218; RYA: 84; SA: 288; SVE: 40, 41, 50, 198, 201, 212, 236; TOM: 114, 115, 117; TVE: 19, 76, 136, 141; TYU: $198^{\mathrm{IG}}$; VGG: 157, 198, 212, 220, 223, 284, 328, 338; VOR: 198.

Didymium nigripes (Link) Fr. - ALT: 299, 301, 302, 306, 308, 310 ${ }^{\mathrm{IG}}$, 315; AS T: 333; CHE: 50, 53, 198; CHU: 196, 210, 212, 290; CR: 30, 174; KDA: 90; KO: 50, 198; KR: 198, 212, 254; KRS: 185; KYA: 120, 122, 123, 124, 198; LEN: 89, 101, 182, 183, 195, 198, 199, 212, 237, 323; LIP: 198, 252; MOS: 4, 6, 7, 9, 63, 64, 66, 70, 84, 164, 198, 278; NVS: 301, 308, 310 ${ }^{\mathrm{IG}}$; PER: 50; PRI: 21, 84, $218^{\mathrm{AGG}}$; PSK: 73; RYA: 125, 321; SAK: 198; SVE: 45, 50, 51, 198, 201, 212, 236; TVE: 6, 19, 76, 136, 142, 180, 182, 198, 204; VGG: 110, 198, 212, 220, 223, 284, 328, 332, 338; VLA: 173; VOR: 171, 248.

Didymium nullifilum (Kowalski) M.L.Farr - BU: 318; ZAB: 26.

Didymium ochroideum G.Lister - ALT: 301, 308; SA: 288; TY: $304^{\mathrm{CF}}$. 
Didymium perforatum Yamash. - ALT: 301, 308; MOS: 66,70 .

Didymium projectile T.N.Lakh. \& K.G.Mukerji VLA: 85.

Didymium proximum Berk. \& M.A.Curtis AL: 198, 215; MOS: 84.

Didymium pseudodecipiens ad int. - KC: 217.

Didymium quitense (Pat.) Torrend - AL: 214, 215 ${ }^{\mathrm{CF}}$; ALT: 316.

Didymium reticulosporum Novozh. \& Zemly. VG G: 207, 280, 282, 324, 325, 329, 330, 331, 334.

Didymium serpula Fr. - ALT: 315; KYA: $120^{\mathrm{CF}}, 123$, 124, 198; LEN: 101, 183, 195, 197, 198, 237, 282, 323; LIP: 198, 252; MOS: 8, 63, 66, 70; NIZ: 176; SMO: 101, 198; TVE: 76; VOR: 249 .

Didymium squamulosum (Alb. \& Schwein.) Fr. \& Palmquist - AL: 134, 215; ALT: 215, 301, 308, 310 $0^{\mathrm{IG}}$, 315, 316, 317; AST: 198, 213, 220, 222, 223, 327, 328,

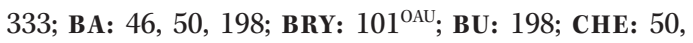
53, 198; CHU: 198, 210; CR: 84, 174, 242, 243, 244; IRK: 198; KDA: 78, 192, 198; KK: 295 ${ }^{\mathrm{IG}}$; KLU: $101^{\text {OAU, KR: }}$ 198, 212, 254; KRS: 5, 198; KYA: 101, 120, 121, 122, 124, 196, 198, 210, 211, 212, 290, 295 ${ }^{\mathrm{IG}}$; LEN: 101, 161, 182, 183, 195, 198, 199, 212, 237, 246, 323; LIP: 198, 252; MAG: 288, 290; MOS: 4, 6, 7, 8, 9, 11, 15, 66, 70, $84,101^{\text {OAU }}, 164$, 198, 238; NIZ: 101, 198; NVS: 301, 308, 310 ${ }^{\mathrm{IG}}$; ORE: 50 , 53, 198, 202; PRI: 21, 74, 84, 218; SA: 288; SAM: 153 , 154; SMO: $101^{\text {OAU }}$, 198; SVE: 46, 50, 53, 198; TOM: 115 ; TVE: $19,76,101^{\text {OAU }}, 136,165,166$; TY: 304; TYU: 84 ; VGG: $110,157,198,212,220,223,281,284,285,328$, 332, 336, 338; VOR: 167, 170; YAN: 196, 198, 210, 290.

Didymium sturgisii Hagelst. - CR: 148, 192.

Didymium trachysporum G.Lister - AST: 198, 213, 220, 221, 222, 223, 328, 333; CR: 174, 243, 244; KL: 198, 328; MOS: 15; SAR: 198; VGG: 110, 157, 198, 220 , 223, 284, 328, 332, 338.

Didymium vaccinum (Durieu \& Mont.) Buchet ORE: 198, 202; VGG: 27, 280.
Echinostelium apitectum K.D.Whitney - ALT: 301, 308, 310 $0^{\mathrm{IG}}$, 316, 317; AST: 333; CR: 241, 243, 244; KDA: 192; KIR: 108, 265; KR: 198; KYA: 122, 124; LEN: 198; MOS: 66, 164; NVS: 301, 308, 310 ${ }^{\mathrm{IG}}$; PRI: 218; VGG: 110, 127, 128, 332, 337; ZAB: 26.

Echinostelium arboreum H.W.Keller \& T.E.Brooks - AST: 213, 220, 222, 223, 328, 333; BA: 50; CR: 243, 244; ORE: 53; VG G: 198.

Echinostelium brooksii K.D.Whitney - AL: 215; ALT: 301, 308, 310 ${ }^{\mathrm{IG}}, 316$; KR: 198; KYA: 122, 124, 196, 198, 210, 211, 212, 290; NVS: 310 ${ }^{\mathrm{IG}}$; SVE: 50, 198; VGG: 128, 282, 338.

Echinostelium colliculosum K.D.Whitney \& H.W.Keller - AL: 214, 215; ALT: 317; AST: 198, 213, 220, 221, 222, 223, 327, 328, 333; CR: 186, 244; ORE: 198, 202; SAR: 198; VGG: 128, 198, 220, 223, 328, 332, 338.

Echinostelium corynophorum K.D.Whitney - AL: 214, 215.

Echinostelium cribrarioides Alexop. - AL: 214, 215; SVE: 198.

Echinostelium elachiston Alexop. - AST: 333; CR: 192, 244; KDA: 192, 198; KIR: 108, 265; KYA: 122, 124; ORE: 53, 198, 202; SVE: 50, 198; VG G: 280.

Echinostelium fragile Nann.-Bremek. - AL: 215; ALT: 215, 310 $0^{\mathrm{IG}}, 316,317$; CR: 244; KYA: 122, 124; NVS: $310^{\text {IG }}$; VG G: $128,282,338$.

Echinostelium lunatum L.S.Olive \& Stoian. - AST: $220^{\mathrm{CF}}, 222^{\mathrm{CF}}$; CR: 192.

Echinostelium microsporum A.Vlasenko - KHM: 314.

Echinostelium minutum de Bary - AL: 214, 215; ALT: $215,301,308,310^{\mathrm{IG}}, 315,316,317$; AST: 198, 213 , 220, 221, 222, 223, 328, 333; BA: 50, 198; BU: 198; CHE: 50, 198; CHU: 196, 198, 210, 212, 290; CR: 186, 192, 241, 242, 243, 244; KDA: 78, 192, 198; KHM: 52; KIR: 274, 275, 276; KO: 50; KR: 130, 198, 212, 254; KYA: 120, 122, 123, 124, 196, 198, 210, 211, 212, 290; LEN: 161, 195, 198, 199, 212, 237; MAG: 288, 290; MOS: 7, 66, 159, 160, 164; MUR: 196, 198, 205, 210, 212, 290; 
NVS: 301, 308, 310 ${ }^{\mathrm{IG}}$; ORE: 50; PER: 50; PRI: 74, 218; PSK: 140; ROS: 147; SAM: 155; SVE: 43, 44, 45, 50, 51, 55, 198, 201, 212, 236; TVE: 19, 76, 136, 165, 166, 179; VGG: $110,128,157,198,220,223,328,332,338$; VOR: 192, 198; YAN: 50, 196, 198, 210, 212, 290.

Echinostelium novozhilovii A.Vlasenko - KHM: 313.

Enerthenema intermedium Nann.-Bremek. \& R.L.Critchf. - PRI: 218.

Enerthenema papillatum (Pers.) Rostaf. - AL: 3, 134, 191, 214, 215; ALT: 301, 308, 310 ${ }^{\mathrm{IG}}$, 317; AST: 333; BA: 50, 198; CHE: 50, 198; CHU: 189, 196, 198, 210, 212, 290; CR: 192, 243, 244; DA: 162; KDA: 90; KHM: 47, 58; KK: $250^{\mathrm{IG}}, 294^{\mathrm{IG}}$; KO: 50, 198; KR: 198, 212, 254; KYA: $101,122,124,196,198,210,211,212,250^{\mathrm{IG}}, 290,294^{\mathrm{IG}}$; LEN: 101, 161, 180, 182, 183, 195, 198, 199, 212, 237, 246; MAG: 198, 290; MOS: 1, 4, 6, 7, 11, 63, 66, 70, 84, 198; MUR: 65, 67, 84; NVS: 301, 308, 310 ${ }^{\mathrm{IG}}$; ORE: 50, 198; PER: 50; PRI: 74, 218; PSK: 140; ROS: 147; RYA: 84, 125, 321; SMO: 101, 198; SVE: 40, 41, 43, 50, 51, 55, 198, 201, 212, 234, 236; TVE: 19, 76, 84, 136, 141, 142, 179, 182, 198, 204; TYU: 84, 198 ${ }^{\mathrm{IG}}$; VGG: 198, 212, 220 , 223, 284, 285, 328; VOR: 170; YAN: 50, 196, 198, 210 , 212, 290.

Fuligo cinerea (Schwein.) Morgan - AL: 3, 134, 198, 215, 300; AST: 198, 213, 220, 221, 222, 223, 327, 328, 333; CR: 244; KIR: 263, 265; KL: 198, 220, 222, 223, 328; LEN: 182, 183, 195, 198, 212, 237; LIP: 198, 252; MOS $^{\mathrm{DR}}$ : 4, 6, 7, 198; RYA: 84; TVE: 136, 204; VGG: 110, 157, 198, 212, 220, 223, 284, 328, 332, 336, 338; VOR: 167.

Fuligo intermedia T.Macbr. - AST: 84.

Fuligo laevis Pers. - BA: 50, 54, 198; SVE: 236.

Fuligo leviderma H.Neubert, Nowotny \& K.Baumann - AL: 215; ALT: 215, 301, 302, 306, 308, 316; BA: 50, 54, 198; DA: 162; IRK: 84; KDA: 90; KHM: 47, 58; KR: 198, 212, 254; MOS: 1, 11, 66, 70, 84; NVS: 301, 308; PRI: 218; PSK: 73; ROS: 147, 335; SVE: 49, 50, 51, 198, 236; TVE: 19, 76, 84, 141, 166; TYU: $84,198^{\mathrm{IG}}$; VGG: 157, 198; VLA: 173.

Fuligo licentii Buchet - NVS: 301, 307, 308.
Fuligo luteonitens L.G.Krieglst. \& Nowotny AL: 215; BA: 54; CHE: 50, 198; MOS: 84; NVS: 301, 307, 308; PSK: 73; SVE: 51; TVE: 19, 76, 141.

Fuligo megaspora Sturgis - KDA: 77, 78.

Fuligo muscorum Alb. \& Schwein. - AL: 133, 134, 215; CHE: 198; KYA: 113; LEN: 180, 182, 183, 195, 199, 237, 323; NVS: 301, 307, 308; PSK: 73; SVE: 49, 50; TOM: 114, 115, 132, 133; TVE: 19, 76, 136, 204.

Fuligo septica (L.) F.H.Wigg. - AD: 339; AL: 3, 134, 191, 215; ALT: 215, 301, 302, 306, 308, 316, 317; ARK: 143, 339; AST: 101, 198, 282, 327, 328, 329, 333, 339; BA: 50, 54, 198; BRY: $16,101^{\mathrm{OAU}}, 111,126$; CHE: 50 , 198; CR: 30, 101, 243, 244; CU: 101 ${ }^{\text {OAU }}$; DA: 162; IRK: 232; KB: 101; KC: 57, 97, 98, 339; KDA: 68, 339; KGD: 91; KGN: 339; KHM: 47, 52, 58; KIR: 263, 264, 265 , 268, 275, 291; KLU: 8, 101, 198; KO: 50, 198, 339; KR: 84, 104, 130, 143, 198, 212, 254, 339; KRS: 5, 101, 185, 198, 339; KYA: $10,120,122,123,124,129,198$; LEN: 89, 96, 101, 180, 182, 183, 195, 198, 199, 212, 237, 245, 286, 287, 323; LIP: 198, 252; MAG: 198; ME: $101^{\mathrm{OAU}}$; MOS: $1,2,4,6,7,8,9,11,18,28,63,64,66,70,84,92$, 94, 101, 158, 164, 175, 198, 278, 289; MUR: 84, 104, 339; NIZ: 176; NVS: 301, 308; ORL: 168; PER: 50; PRI: 21, 22, 177, 218; PSK: 73, 84, 140, 339; ROS: 147; RYA: 84, 125, 321; SA: 339; SAM: $101^{\text {OAU; }}$ SE: 198, 339; SMO: 99, 100, 101, 198; STA: 57, 97, 98, 101, 198; SVE: 40, $41,42,45,49,50,51,198,201,212,236$; TA: $101^{\mathrm{OAU}}$, 198, 262; TOM: 114, 132, 198, 233; TVE: 6, 19, 76, 84, $101^{\text {OAU }}, 135,136,139,141,142,166,179,180,182,198$, 204, 296; TYU: 84, 198 ${ }^{\mathrm{IG}}$, 339; ULY: $101^{\text {OAU }}$; VGG: 198 , 257, 280, 281, 284, 285, 328, 338; VLA: 173; VOR: 101, 167, 170, 171, 198, 239, 248, 249, 339; ZAB: 105.

Hemitrichia abietina (Wigand) G.Lister - AL: 215; BA: 50, 198; CR: 243, 244; KHA: 198; KYA: 196, 198, 210, 211, 212, 290; MOS: 66, 70, 158; NVS: 301, 308; PRI: 74, 218; SVE: 50, 198, 212, 236; TYU: 84; VGG: 157, 198.

Hemitrichia calyculata (Speg.) M.L.Farr - AL: 3, 215; ALT: 215, 301, 308, 316; BA: 50, 198; CR: 144, 243, 244; KIR: 276, 277; MOS: 6, 7, 11, 64, 84; NVS: 301, 308; PRI: 69, 74, 84, 218; PSK: 73; ROS: 147; RYA: 84; TVE: 19, 76, 141; TYU: 84; VGG: 338. 
Hemitrichia chrysospora (Lister) Lister - MOS: 7.

Hemitrichia clavata (Pers.) Rostaf. - AL: 3, 133, 134, 191, 215; ALT: 215, 301, 302, 306, 308, 316; BA: 50, 54, 198; BEL: 12; BRY: 16, $101^{\text {OAU }}$; CHE: 50; CR: 144,241 , 244; IRK: 84; KDA: 90, 192, 198; KHA: 84, 198; KHM: 47, 52, 58; KIR: 269; KK: $295^{\mathrm{IG}}$; KLU: $8,101^{\text {OAU; }}$ KO: 50 , 198; KR: 198, 212, 254; KRS: 101, 185, 198; KYA: 101, 122, 124, 198, $295^{\mathrm{IG}}$; LEN: 101, 180, 182, 183, 195, 198, 199, 212, 237, 323; LIP: 198, 252; MOS: 1, 4, 6, 7, 8, 9 , 64, 66, 70, 84, 101, 159, 160, 164, 198, 278; MUR: 65, 196, 205, 210, 212, 290; NVS: 301, 308; ORE: 50, 198; PER: 50; PRI: 21, 74, 203, 218; PSK: 73; ROS: 147, 335; RYA: 84, 125, 321; SMO: 101 ${ }^{\text {OAU }}$, 198; STA: 198; SVE: 40, 41, 42, 49, 50, 51, 198, 201, 212, 236; TA: 101, 198, 262; TOM: 132,198 ; TVE: $19,76,84,101^{\text {OAU }}, 136,141$, 180, 182, 198, 204; TYU: 84, $198^{\mathrm{IG}}$; VG G: 157, 198, 212 , 220, 223, 281, 328, 336, 338; VLA: 173.

\section{Hemitrichia cornuvioides Lavrov - TOM: 133.}

Hemitrichia intorta (Lister) Lister - KIR: 108, 265; LEN: 182, 195, 212, 237; MOS: 6, 7, 8, 9, 66, 70, 164, 198, 278; TVE: 19, 79, 80, 136, 180, 181, 182, 282; VGG: 127, 220, 223, 328, 337.

Hemitrichia leiocarpa (Cooke) Lister - KRS: 12; MOS $^{\mathrm{DR}}$ : 6, 7, 198; VGG: 198, 220, 223.

Hemitrichia leiotricha (Lister) G.Lister - ALT: 317; KO: 50, 198; KRS: 5, 198; MAG: $288^{\mathrm{CF}}$; MOS: 6, 7, 8, 66, 164, 198; SVE: 50, 198, 201, 236.

Hemitrichia minor G.Lister - AST: 220, 222, 223; CHE: 50, 53, 198; CR: 242, 243, 244; KIR: 108, 265; KR: 198, 212, 254; LEN: 198; MAG: 290; MOS: 6, 7, 8, 66, 70, 164, 198; ORE: 53, 198, 202; PRI: 74, 218 ; SVE: 46, 50, 53, 212; VGG: 198, 220, 223.

Hemitrichia pardina (Minakata) Ing - AL: 215; ALT: 215, 301, 308, 315, 316, 317; AST: 328; KR: 198; KYA: 120, 122, 123, 124, 198; LIP: 198; MAG: 288; NVS: 301, 308; ORE: 198, 202; SA: 288; SAM: 155; SVE: 201; TVE: 19, 76, 165, 166; VGG: 110, 328, 332, 338.

Hemitrichia serpula (Scop.) Rostaf. ex Lister - AL: 3, 134, 191, 215; ALT: 215, 301, 308, 315, 316, 317; BA: 50, 54, 198; CHE: 50, 198; IRK: 84; KDA: 192, 198; KHA:
198; KIR: 108, 265, 267, 268, 269, 275, 276, 277; KLU: 8; KYA: 10, 122, 124, 198; LEN: 101, 161, 182, 183, 195, 197, 198, 212, 237, 323; Mos: 6, 7, 9, 64, 66, 70, 84, 164, 198, 278; NVS: 301, 308; ORL: 198; PRI: $21,22,74,84$, 203, 218; PSK: 73, 140, 198; SMO: 101, 198; SVE: 50, 198, 201, 212, 236; TVE: 19, 76, 84, 136, 141, 198, 204; TY: 304; TYU: 84; VGG: 110, 332; VOR: 171, 239, 248, 249.

Kelleromyxa fimicola (Dearn. \& Bisby) Eliasson AL: 37, 214, 215, 318; ALT: 27, 318; AST: 198, 213, 220, 222, 223, 328, 329, 333; BU: 305, 318; NVS: 318; ORE: 53.

Lamproderma aeneum Mar.Mey. \& Poulain - KAM: 219; KC: 34, 217; KR: 34; LEN: 35.

Lamproderma arcyrioides (Sommerf.) Rostaf. ALT: 301, 308; AST: 198, 220, 223, 327, 328, 333; CHU: 196, 198, 210, 212, 290; KC: 34, 217; KO: 50, 198; KR: 34, 35, 198; KRS: 185, 198; KYA: 122, 124; LEN: 34, 35, 182, 183, 188, 195, 198, 212, 237, 261; LIP: 198, 252; MOS: 6, 7, 64, 66, 70, 198, 278; MUR: 34, 36, 196, 198, 205, 210, 212, 290; PRI: 74, 203; TA: 101, 198; TVE: 19 , 20, 76, 136, 141; TYU: 84; VGG: 284.

Lamproderma cacographicum Bozonnet, Mar. Mey. \& Poulain - KC: 34, 217; MUR: 34, 36.

Lamproderma carpatiensis ad int. - LEN: 261.

Lamproderma columbinum (Pers.) Rostaf. - AL: 191, 215; BU: 198; KHA: 198; KHM: 47, 58; KO: 50, 198; KR: 143, 198, 212, 254; LEN: 101, 182, 183, 195, 198, 199, 212, 237, 323; LIP: 198, 252; MOS: 1, 7, 9, 66, 70, 84, 198; MUR: 104; NVS: 301, 308; PER: 50; PRI: 74, 203, 218; PSK: 140; SMO: 101, 198; SVE: 50, 51, 198, 236; TA: 101, 198, 262; TVE: 19, 76, 136, 141, 142, 198, 204; TYU: 198 ${ }^{\mathrm{IG}}$; VGG: 220, 223, 328.

Lamproderma cristatum Meyl. - KC: 34, 217.

Lamproderma cucumer (Meyl.) Nowotny \& H.Neubert - KAM: 219; KC: 34, 217.

Lamproderma disseminatum Kowalski - KHM: 47, 58; TYU: $198^{\mathrm{IG}}$.

Lamproderma echinosporum Meyl. - KAM: 219; KC: 34, 217. 
Lamproderma echinulatum (Berk.) Rostaf. - MOS $^{\mathrm{DR}}$ : $6,198$.

Lamproderma gulielmae Meyl. - KR: 198, 212, 254; TVE: 19.

Lamproderma kowalskii A.Ronikier, Lado \& Mar. Mey. - MUR: 34, 36 ${ }^{\mathrm{CF}}$.

Lamproderma laxum H.Neubert - SVE: 236.

Lamproderma maculatum Kowalski - KAM: 219; KC: 34, 217; MUR: 34, 36; TVE: 20.

Lamproderma ovoideum Meyl. - BA: 54; DA: 162 ${ }^{\mathrm{CF}}$; KAM: 219; KC: 34, 217, 258; MUR: 34, 36; TVE: 20.

Lamproderma pseudomaculatum Mar.Mey. \& Poulain - KAM: 219; KC: 34; MUR: 34, 36.

Lamproderma pulchellum Meyl. - KC: 34, 217; MUR: 34, 36.

Lamproderma pulveratum Mar.Mey. \& Poulain KAM: 219; KC: 34, 216, 217, 255; MUR: 34, 36.

Lamproderma sauteri Rostaf. - BA: 54; KAM: 219; KC: 34, 217; KHM: 47, 58; KR: 34, 198, 212, 254; KYA: 196, 198, 210, 211, 212, 290; LEN: 35, 261; MUR: 34, 36, 196, 198, 205, 210, 212, 290.

Lamproderma scintillans (Berk. \& Broome) Morgan - AL: 215; ALT: 215, 301, 308, 315, 316, 317; CHE: 50, 53, 198; CR: 244; KDA: 78; KLU: 8; KO: 50, 198; LEN: 182, 183, 195，198，199, 212, 237; MAG: 290; MOS: 7, 8, 15, 66, 70, 84, 164; MUR: 65, 96; NVS: 301, 308; PRI: 74, 203, 218; PSK: 140; SA: 288; TVE: 19, 76, 141; VGG: 157, 198, 212, 220, 223, 328.

Lamproderma spinulosporum Mar.Mey., Nowotny \& Poulain - KC: 34, 217, 258; KR: 34, 35; LEN: 34, 35, 258; MUR: 34, 36.

Lamproderma splendens Meyl. - KAM: 219; KC: 34, 217; TVE: 20.

Lamproderma splendidissimum Mar.Mey., Bozonnet \& Poulain - KC: 217.
Lamproderma zonatum Mar.Mey. \& Poulain - KR: 34; LEN: 35; TVE: 20.

Leocarpus fragilis (Dicks.) Rostaf. - AL: 3, 134, 214, 215; ALT: 299, 301, 302, 306, 308, 316; BRY: $101^{\mathrm{OAU}}$, 111, 126; BU: 198; CHU: 196, 198, 210, 212, 290; CR: 30, 84; IRK: 198; IVA: 198; KAM: 219; KDA: 90; KHM: 47, 52, 58, 196; KIR: 267, 268, 269, 275, 291; KO: 50, 198; KR: 130, 198, 212, 254; KYA: 10, 113, 120, 122, 123, 124, 196, 198, 210, 211, 212, 290; LEN: 89, 101, 180, 182, 183, 195, 198, 199, 212, 237, 261, 323; LIP: 198, 252; MAG: 198, 290; MOS: 1, 4, 6, 7, 8, 9, 11, 63, 64, 66, 70, 84, 94, 95, 101, 158, 164, 175, 198, 278; MUR: 65, 205, 210, 212, 290; NIZ: 176; PER: 50, 198; PRI: 21, 218; PSK: 73, 140; ROS: 335; RYA: 84, 125, 321; SA: 288; SAM: 154, 156; SMO: 101, 198; STA: 198; SVE: 40, 41, 49, 50, 51, 198, 201, 212, 236; TA: 101, 198, 262; TOM: 114, 115, 132, 198; TVE: 6, 19, 76, 84, 135, 136, 141, 179, 180, 182, 198, 204; TYU: 198 ${ }^{\mathrm{IG}}$; VGG: 198, 220, 223, 328; VLA: 173; VOR: 248; YAN: 196, 198, 210, 212, 290; YAR: 6, 84, 198; ZAB: 26.

Lepidoderma alpestroides Mar.Mey. \& Poulain MUR: 34, 36.

Lepidoderma carestianum (Rabenh.) Rostaf. CHU: 196, 198, 210, 212, 290; KAM: 219; KC: 34, 217, 255, 258, 259; LEN: 34, 35, 188, 195, 197, 212, 237, 282; MOS: 8, 66 ${ }^{\mathrm{CF}}$; MUR: 34, 36, 67, 259.

Lepidoderma chailletii Rostaf. - KAM: 219; KC: 34, 217, 255, 258, 259; KR: 34, 35, 259; LEN: 34, 35, 259, 261; MUR: 34, 36, 67, 196, 198, 205, 210, 212, 258, 259 , 290; TVE: 20.

Lepidoderma crassipes Flatau, Massner \& Schirmer - KHM: $47^{\mathrm{CF}}, 58^{\mathrm{CF}}$; TYU: $198^{\mathrm{IG}}$.

Lepidoderma granuliferum (W.Phillips) R.E.Fr. KC: 34, 217; MUR: 34, 36, 196, 198, 205, 210, 212, 290; ZAB: 26.

Lepidoderma peyerimhoffii Maire \& Pinoy - KC: 34, 217, 255, 258.

Lepidoderma tigrinum (Schrad.) Rostaf. - AL: 215; BA: 54; KDA: 78; KHM: 47, 52, 58; KO: 50, 198; KR: 198, 212, 254; KYA: 122, 124; LEN: 101, 182, 183, 195, 
197, 198, 199, 212, 237, 261, 282, 323; MOS: 6, 8, 66, 84, 198; MUR: 65, 67; PER: 50; PSK: 140; SVE: 50, 51, 198; TVE: $19,76,136,141,142$; TYU: $198^{\mathrm{IG}}$.

Lepidoderma trevelyanii (Grev.) Poulain \& Mar. Mey. - BA: 50, 198; KR: 198, 212, 254; LEN: 101, 183, 195, 197, 198, 212, 237, 282, 323; NVS: 301, 307, 308.

Leptoderma iridescens G.Lister - AL: 134; ALT: 301, 307; MOS: 164; TOM: 132, 198.

Licea belmontiana Nann.-Bremek. - ALT: 317; AST: 198, 213, 220, 221, 222, 223, 327, 328, 333; CHU: 196 198; CR: 148, 187, 192, 244; KDA: 192; KIR: 108, 265; KLU: 8; KO: 50, 198; KYA: $196^{\mathrm{CF}}, 198,211,212^{\mathrm{CF}}, 290^{\mathrm{CF}}$; MOS: 7, 8, 66, 164; MUR: 65; PER: 50; PRI: 218; SVE: 198, 236; TVE: 19, 76, 166; VGG: 127, 284, 332, 337; VOR: 192 ; YAN: $50,196^{\mathrm{CF}}, 198,212^{\mathrm{CF}}, 290^{\mathrm{CF}}$.

Licea biforis Morgan - ALT: 301, 308, 315, 316, 317; AST: 333; BA: 50, 198; CR: 187, 192; KDA: 78, 192, 198; KYA: 122, 124; MAG: 290; MOS: 66, 164; PRI: 218; SVE: 50, 201, 212; TVE: 136, 165, 166, 179, 198, 204; VGG: 127, 157, 198, 220, 223, 281, 284, 328, 337.

Licea castanea G.Lister - ALT: 317; BA: 198; CR: 192, 244; KDA: 192, 198; KR: 198, 212, 254; KYA: 120, 122, 123, 124, 198; LEN: 195, 198, 212, 237; MOS: 7, 66; MUR: 65, 67; PER: 50; PRI: 218; SVE: 198; TVE: 19; VGG: 110, 157, 332; VOR: 167, 170, 192, 198, 239, 249.

Licea chelonoides Nann.-Bremek. - AST: 220, 222, 223, 328; ВА: 50, 198; KНM: 47, 58; KL: 328; KO: 198; PER: 50; SVE: 198, 236; TYU: $198^{\mathrm{IG}}$; VGG: 328.

Licea denudescens H.W.Keller \& T.E.Brooks - AST: 198, 213, 220, 221, 222, 223, 327, 328, 333; KR: 198; KYA: $210^{\mathrm{CF}}$; LEN: 198; MOS: 7, 66; VGG: 110, 332, 337.

Licea erecta K.S.Thind \& Dhillon - ALT: 317.

Licea inconspicua T.E.Brooks \& H.W.Keller - Mos: 66.

Licea iridis Ing \& McHugh - BA: $50^{\mathrm{CF}}$.

Licea kleistobolus G.W.Martin - AL: 215; ALT: 215, 301, 308, 310 ${ }^{\mathrm{IG}}$, 316, 317; AS T: 198, 213, 220, 222, 223, 327, 328, 333; BA: 50, 198; CHE: 50, 198; CHU: 196,
198, 210, 212, 290; CR: 174, 187, 192, 243, 244; KDA: 78, 192; КНM: 26; KR: 198, 212, 254; KYA: 120, 121, 122, 123, 124, 196, 198, 210, 211, 212, 290; LEN: 161, 195, 198, 199, 212, 237; MOS: 7, 15, 66, 159, 160, 164; NVS: 301, 308, 310 ${ }^{\mathrm{IG}}$; PRI: 218; ROS: 198, 220, 223; SAM: 155; SVE: 46, 48, 50, 55, 198, 235; TVE: 19, 76, 165, 166; VGG: 157, 198, 220, 223, 328, 338; YAN: 50, 196, 198, 210, 212, 290; ZAB: 26.

Licea marginata Nann.-Bremek. - LEN: 195, 198, 199, 212, 237.

Licea minima Fr. - AL: 191, 215; B A: 50, 54, 198; CHE: 50, 198; CHU: 196, 198, 210, 212, 290; CR: 174, 241, 243, 244; КНM: 47, 52, 58; KLU: 8; KO: 50, 198; KR: 130, 198, 212, 254; KYA: 122, 124, 196, 198, 210, 211, 212, 290; LEN: 161, 195, 198, 199, 212, 237; MOS: 7, 8, 66, 70, 84; MUR: 65, 104, 205, 210, 212, 290; PER: 50; PRI: 218; ROS: 147; SVE: 42, 43, 44, 49, 50, 51, 55, 198, 201, 212, 236; TVE: 19, 76, 141, 166; TYU: 84, $198^{\mathrm{IG}}$; VGG: 198, 220, 223, 280, 328; YAN: 50, 196, 198, 210, 212, 290.

Licea nannengae Pando \& Lado - AST: 198, 213, 220, 222, 223, 328; KL: 198, 220, 222, 223, 328; VGG: 110, 198, 220, 223, 284, 328, 332.

Licea operculata (Wingate) G.W.Martin - ALT: 301, 308, 316; BA: 50, 198; CHE: 50, 198; CHU: 196, 198, 210, 212, 290; CR: 174, 187, 192, 244; KDA: 192, 198; KIR: 275, 276; KRS: 185, 198; KYA: 118, 119, 120, 122, 123, 124, 198; LEN: 161, 195, 198, 199, 212, 237; LIP: 198; MOS: 7, 66, 84, 159, 160, 164; NVS: 301, 308; ORE: 198, 202; PRI: 74, 218; ROS: 198, 220, 223; SAM: 155; SAR: 198; TVE: 19, 76, 165, 166; VGG: 198, 220, 223, 328; YAN: 50, 196, 198, 210, 212, 290.

Licea parasitica (Zukal) G.W.Martin - AL: 214, 215; ALT: 301, 308, 310 ${ }^{\mathrm{IG}}$; AST: 213, 220, 222, 223, 328; BA: 198; CHE: 198; CHU: 196, 198, 210, 212, 290; CR: 174, 187, 192, 244; KDA: 198; KIR: 276; KR: 212, 254; KRS: 198; KYA: 120, 122, 123, 124, 198; LEN: 195, 198, 199, 212, 237; LIP: 198; MOS: 7, 66, 164; NVS: 301, 308, 310 ORE: 198; PRI: 74, 218; ROS: 198; SAR: 198; TVE: 19, 76, 166; VGG: 110, 198, 220, 223, 284, 328, 332, 338; VOR: 167, 170, 192; YAN: 50, 196, 198, 210, 212, 290.

Licea pedicellata (H.C.Gilbert) H.C.Gilbert - ALT: 315, 317; PRI: 218. 
Licea pusilla Schrad. - ALT: 315, 317; AST: 220, 222, 223, 328; BA: 54; BU: 198; CR: 148; KDA: 78; KHM: 26; KO: 50, 198; KR: 130; KYA: 122, 124; LEN: 180, 181, 182, 183, 195, 198, 212, 237, 323; MOS: $6,7^{\mathrm{CF}}, 8,15$, 66, 70, 198; PER: 50; PRI: 22, 74, 218; SMO: 101, 198; SVE: $42^{\mathrm{CF}}, 44^{\mathrm{CF}}, 50,198,236$; TVE: $19,76,136,142$, 165; VGG: 127, 280, 332, 337; VOR: 248.

Licea pygmaea (Meyl.) Ing - AL: 215; PRI: 218; SVE: 236; TVE: 19, 141.

Licea rugosa Nann.-Bremek. \& Y.Yamam. - PRI: 218.

Licea scintillans McHugh \& D.W.Mitch. - CR: 148.

Licea scyphoides T.E.Brooks \& H.W.Keller - VG G: 223.

Licea tenera E.Jahn - AL: 214, 215; ALT: 316, 317; KRS: 185,198 ; KYA: 120, 121, 122, 123, 124, 198; MOS $^{\mathrm{DR}}$ : 6, 7, 8, 164, 198, 278; PER: 50; SVE: 198; TVE: 136, 165, 166, 179, 198, 204; VGG: 198, 220, 223, 284, 328.

Licea testudinacea Nann.-Bremek. - AL: 214, 215; ALT: 301, 308, 317; BA: 54; CHE: 50, 198; KO: 50, 198; KR: 198; KRS: 198; KYA: 122, 124, 196, 198, 210, 211 , 212, 290; LEN: 198; MOS: 7, 66; MUR: 65; PRI: 218; SVE: 50, 198, 201, 212; TVE: 19, 76, 141; VGG: 198, $220,223,328$.

Licea variabilis Schrad. - AL: 3, 134, 215, 300; ALT: 301, 308, 317; CR: 174, 243, 244; KHM: 47, 52, 58; KLU: 101, 198; KR: 198, 212, 254; LEN: 182, 183, 195, 198, 199, 212, 237; MOS: 6, 7, 8, 66, 70, 198, 278; PRI: 218; SMO: 101, 198; SVE: 50, 198, 201, 212, 236; TVE: 136, 198, 204; TYU: 198 ${ }^{\mathrm{IG}}$; YAN: 50, 196, 210, 212, 290.

Licea verrucispora D.Wrigley \& Lado - PRI: 218.

Lindbladia tubulina Fr. - KDA: 198; KO: 50, 198; KR: 198, 212, 254; KYA: 10, 198; LEN: 101, 182, 183, 195, 197, 198, 199, 212, 237, 282; LIP: 198, 252; MAG: 290; MOS: 6, 7, 66, 70, 198, 278; PRI: 21; SMO: 198; TA: 101, 198; TVE: 84, 136, 198, 204; TYU: $198^{1 \mathrm{IG}}$; VLA: $172,173$.

Listerella paradoxa E.Jahn - VOR: 32 .

Lycogala confusum Nann.-Bremek. ex Ing - Mos: 63.
Lycogala conicum Pers. - KDA: 68; KIR: 268; MOS: 1, 63, 64, 66, 70, 138; PRI: 218; TVE: 79, 80, 136, 138, 198, 204, 282; VLA: 172.

Lycogala epidendrum (L.) Fr. - AL: 3, 134, 191, 214 , 215, 233; ALT: 134, 215, 299, 301, 302, 306, 308, 316, 317; ARK: 143; AST: 101, 198; BA: 50, 54, 198; BEL: 12; BRY: 16, $101^{\text {OAU }}$, 126; CHE: 50; CHU: 189, 196, 198, 210, 212, 290; CR: 84, 101, 150, 243, 244; DA: 162; IRK: 84, 232; KAM: 198, 251, 297; KC: 57, 97, 98; KDA: 68, 192, 198; KGD: 91; KGN: $101^{\mathrm{IG}}$; KHA: 84, 198; KHM: 47, 52, 58; KIR: 14, 263, 264, 265, 266, 268, 271, 272, 274, 275, 276, 277, 291; KK: 292 ${ }^{\mathrm{IG}}$; KLU: 8, 101, 198; KO: 50, 198; KR: 84, 104, 130, 198, 212, 254; KRS: 5, 101, 185, 198; KYA: $10,101,103,112,113,120,122$, 123, 124, 129, 196, 198, 210, 211, 212, 290, $292^{\mathrm{IG}}$; LEN: 62, 87, 89, 101, 180, 182, 183, 195, 198, 199, 212, 237 , 286, 287, 323; LIP: 198, 252; MAG: 198, 290; MOS: 1 , $4,6,7,8,9,11,18,62,63,64,66,70,84,101,158,159$, 160, 164, 175, 198, 278, 289; MUR: 65, 84, 104, 106, 205, 210, 212, 290; NIZ: 176; NVS: 198, 233, 301, 308; ORE: 50, 198; ORL: 168; PER: 50; PRI: 21, 22, 69, 74, 84, 177, 203, 218; PSK: 73, 101, 140, 198; ROS: 101, 147, 198, 220, 223, 335; RYA: 84,125 , 321; SA: 231, 288, 298, 326; SAM: $153,155,156$; SMO: 99, 100, 101 ${ }^{\text {OAU }}$, 198; STA: 101, 198; SVE: 39, 40, 41, 42, 43, 45, 49, 50, 51, $101^{\mathrm{IG}}$, 198, 201, 212, 234, 236; ТА: 101, 198, 262; тОМ: 114 , 132, 198, 233; TVE: 19, 76, 84, 101, 135, 136, 139, 141, 142, 166, 179, 180, 182, 198, 204, 296; TYU: $84,101^{\mathrm{IG}}$, $198^{\mathrm{IG}}$; VG G : 157, 198, 212, 220, 223, 281, 284, 328, 336, 338; VLA: 6, 84, 173, 198; VOR: 167, 171, 239, 248, 249; YAN: 196, 198, 210, 212, 290; ZAB: 101, 105, 198.

Lycogala exiguum Morgan - AL: 214, 215; ALT: 215; AST: 333; BA: 50, 198; CHE: 50, 198; KDA: 90; KHA: 198; KHM: 47, 52, 58; KIR: 264, 265, 266, 271; KO: 50, 198; KRS: 5, 198; LEN: 180, 182, 183, 195, 198, 212, 237; Mos: 1, 4, 6, 7, 8, 9, 11, 63, 64, 66, 70, 84, 164, 198; MUR: 84; PER: 50; PRI: 69, 74, 84, 203, 218; PSK: 73; RYA: 125, 321; SVE: 49, 50, 51, 198, 201, 236; TVE: 19, 76, 84, 136, 141, 179, 180, 182, 198, 204; TYU: 84 , 198 ${ }^{\mathrm{IG}}$; VG G: 198, 220, 223, 280, 284, 328; VLA: 173.

Lycogala flavofuscum (Ehrenb.) Rostaf. - AL: 3, 215, 300; AST: 198, 220, 223, 327, 328, 333; BA: 198; BEL: 12; CHE: 198; IRK: 232; KHA: 198; KIR: 276, 277; KO: 198; KR: 130; KRS: 101, 185, 198; KYA: 10, 122, 124, 198; LEN: 89, $101^{\text {OAU }}, 195,198,212,237$; MOS: $4,7,8$, 
18, 66, 70, 175; NGR: 101 ${ }^{\mathrm{OAU}}$; NVS: 301, 308; PRI: 21, 22, 218; PSK: 73, $101^{\mathrm{OAU}}$; ROS: 335; SMO: 101; SVE: 198, 236; TA: 101, 198, 262; TVE: 136, 198, 204; VGG: 198, 212, 220, 223, 328, 338.

Macbrideola cornea (G.Lister \& Cran) Alexop. ALT: 317; BA: 50, 198; CR: 190, 192, 198, 241, 243, 244; KDA: 78, 190, 192, 198; KIR: 276; KR: 198, 212, 254; KYA: 196, 198, 210, 211, 212, 290; LEN: 161, 195, 198, 199, 212, 237; MOS: 66, 84, 159, 160, 164; PRI: 218; SVE: 212; TVE: 19, 76, 166; VGG: 198, 220, 223, 328, 337; YAN: 50, 196, 198, 210, 212, 290.

Macbrideola decapillata H.C.Gilbert - CR: 190, 241; KDA: 190, 192; KYA: 210; PRI: 218.

Macbrideola martinii (Alexop. \& Beneke) Alexop. - PRI: 218.

Macbrideola oblonga Pando \& Lado - AL: 214, 215; AST: 198, 213, 220, 221, 222, 223, 328, 333; SAR: 198; VGG: $60,337$.

Macbrideola synsporos (Alexop.) Alexop. - AL: 215; CR: 190, 192; KDA: 78, 190; VGG: 27, 337.

Meriderma aggregatum ad int. - KC: 34, 217; KR: 34, 35; LEN: 34, 35; MUR: 34, 36; TVE: 20.

Meriderma carestiae (Ces. \& De Not.) Mar.Mey. \& Poulain - KC: 34, 216, 217, 258; KR: 34, 35; LEN: 34, 35, 258; MUR: 34, 36, 67, 196, 205, 210, 212, 290; TVE: 20.

Meriderma cribrarioides (Fr.) Mar.Mey. \& Poulain - AL: 3, 215, 300; CR: 29, 30; KC: 34, 217; KR: 198; LEN: 182, 183, 195, 198, 237, 261; MOS: $7^{\mathrm{CF}}, 66,70$; TVE: 20 .

Meriderma echinulatum (Meyl.) Mar.Mey. \& Poulain - KC: 34, 217; KR: 34, 35; LEN: 34, 35, 261; MUR: $34,36$.

Meriderma fuscatum (Meyl.) Mar.Mey. \& Poulain MUR: $34^{\mathrm{CF}}, 36^{\mathrm{CF}}, 196,205,210,212,290$.

Meriderma spinulisporum ad int. - KC: 34, 217; KR: 34; LEN: 35; MUR: 34, 36; TVE: $20^{\mathrm{CF}}$.
Meriderma verrucosporum ad int. - KC: 34, 217.

Metatrichia floriformis (Schwein.) Nann.-Bremek. - AL: 215; ALT: 301, 308; IRK: 84; KIR: 268, 277; KO 50, 198; KYA: 116, 120, 122, 123, 124, 198; LEN: 182 , 183, 195, 197, 212, 237; MAG: 288; MOS: 7, 8, 11, 63 , 64, 66, 70, 84, 164; NVS: 301, 308; PRI: 218; PSK: 73; SVE: 50, 51, 198, 201, 212, 236; TA: 262; TVE: 19, 76 , 136, 141, 142, 198, 204, 282; VGG: 338.

Metatrichia rosea (Flatau \& Nann.-Bremek.) Nann.-Bremek. - SVE: 50, 51, 236.

Metatrichia vesparia (Batsch) Nann.-Bremek. ex G.W.Martin \& Alexop. - AL: 3, 134, 191, 215; ALT: 215, 299, 301, 302, 306, 308, 316, 317; AST: 198, 220, 223, 327, 328, 333; BA: 50, 54, 198; BRY: $101^{\mathrm{OAU}}, 111$, 126; CHE: 50, 198; CR: 30, 174, 243, 244; DA: 162; IRK: 84; KAM: 251; KDA: 192, 198; КНА: 84, 198; KHM: 47, 52, 58; KIR: 107, 108, 265, 268, 269, 273, 275, 276, 277; KLU: 8, 101, 198; KO: 50, 198; KRS: 5, 185, 198; KYA: 10, 120, 122, 123, 124, 198; LEN: 89, 101, 180, 182, 183, 195, 198, 199, 212, 237, 323; LIP: 198, 252; MOS: 1, 4, $6,7,8,9,11,18,63,64,66,70,84,101,158,159,160$, 164, 175, 198, 278; MUR: 67; NGR: 101, 198; NVS: 301, 308; PER: 50; PRI: 69, 74, 84, 203, 218; PSK: 73, 101, 140, 198; ROS: 147, 335; RYA: 84, 125, 321; SAM: 154 , 155; SE: 198; SMO: 100, 101 ${ }^{\mathrm{OAU}}$, 198; STA: 198; SVE: 40 , 41, 42, 49, 50, 51, 198, 201, 212, 236; TA: 101, 198, 262 TOM: $114,115,132,198$; TVE: $19,76,79,84,101^{\mathrm{OAU}}$, 136, 139, 141, 142, 166, 180, 182, 198, 204; TYU: 84 $198^{\mathrm{IG}}$; ULY: 101, 198; VGG : 157, 198, 212, 220, 223, 284 , 328, 338; VLA: 173, 198; VOR: 167, 239, 249; ZAB: 198

Mucilago crustacea P.Micheli ex F.H.Wigg. - AL: 3, 134, 215, 300; ALT: 215, 301, 308, 316; ARK: 143; BA: 101 ${ }^{\mathrm{IG}}$, 198; BRY: 111; BU: 198; CE: 198; CHE: $101^{\mathrm{IG}}$; CHU: 196, 198, 210, 212, 290; CR: 30, 84, 242, 243, 244; KAM: 219; KDA: 68; KIR: 265, 267, 268, 269, 275, 276, 277 , 291; KLU: 101, 198; KO: 198; KR: 104, 130; KRS: 5, 101, 185, 198; KYA: 10, 120, 122, 123, 124, 196, 198, 210 , 211, 212, 290; LEN: 96, 101, 182, 183, 195, 198, 199 , 212, 237, 246, 323; LIP: 198, 252; MAG: 198, 290; MOS: 4, 6, 7, 9, 63, 66, 70, 84, 101, 158, 164, 175, 198, 278; MUR: 84, 104; ORE: $101^{\mathrm{IG}}$, 198; ORL: 168, 198; PER: 50 ; PRI: 21, 22, 84, 218; ROS: 198, 220, 223, 335; RYA: 84, 125, 321; SAM: 154, 156; SMO: 101, 198; STA: 198; SVE: 50, 198; TA: 101, 198, 262; TAM: 101, 198; TOM: 117 , 
132, 198; TVE: 19, 76, 135, 136, 141, 142, 182, 198, 204; VGG: $157,198,212,220,223,281,284,285,328,336$, 338; VLA: 173; VOR: 167, 170, 171; YAN: 50, 196, 198, 210, 212, 290.

Oligonema flavidum (Peck) Peck - LEN: 182, 183, 195, 198, 212, 237; MUR: 65; PSK: 73; ROS: 147; VGG: 198, 212, 220, 223, 283, 328, 330.

Oligonema fulvum Morgan - MOS: 66; SVE: 50

Oligonema schweinitzii (Berk.) G.W.Martin - ALT: 317; LEN: 182, 183, 195, 198, 212, 237; MOS: 8; ROS: 147; SMO: 101, 198; VGG: 198, 212, 220, 223, 284, 328, 336.

Paradiacheopsis acanthodes (Alexop.) Nann.Bremek. - KYA: 210.

Paradiacheopsis cribrata Nann.-Bremek. - KYA: 196 ${ }^{\mathrm{CF}}, 198,210,211^{\mathrm{CF}}, 212$, 290; LEN: 198; SMO: 198; SVE: 50, 55, 198, 201, 212; TVE: 19, 76; VGG: 198, 220 , 223,328 .

Paradiacheopsis fimbriata (G.Lister \& Cran) Hertel ex Nann.-Bremek. - AL: 214, 215; ALT: 215, 301, 308, 310 ${ }^{\mathrm{IG}}$, 316; CHE: 50, 198; CHU: 196, 198, 210, 212 , 290; CR: 241, 243, 244; KDA: 78; KO: 50; KR: 198, 212 , 254; KYA: 122, 124, 196, 198, 210, 211, 212, 290; LEN: 161, 195, 198, 199, 212, 237; LIP: 198, 252; MAG: 290; MOS: 7, 66, 164; MUR: 65; NVS: 301, 308, 310 ${ }^{\mathrm{IG}}$; PRI: 218; ROS: 147; SAM: 155; SVE: 42, 43, 44, 45, 50, 51, 53, 55, 198, 201, 212, 236; TVE: 19, 76, 166; YAN: 50, 196, 198, 210, 212, 290; ZAB: 26.

Paradiacheopsis longipes Hooff \& Nann.-Bremek. - KR: 198; MUR: 65.

Paradiacheopsis microcarpa (Meyl.) D.W.Mitch. ex Ing - ROS: $147^{\mathrm{CF}}$; SVE: 50, 198.

Paradiacheopsis rigida (Brândză) Nann.-Bremek. - AL: 198, 215; ALT: 301, 308, 310 ${ }^{\mathrm{IG}}$, 317; CR: 148; KDA: $78^{\mathrm{CF}}$; KYA: 122, 124; MOS: 164; NVS: 301, 308, 310 ${ }^{\mathrm{IG}}$; PRI: 218; TVE: 19; VGG: 27.

Paradiacheopsis solitaria (Nann.-Bremek.) Nann.Bremek. - ALT: 301, 308, 310 ${ }^{\mathrm{IG}}$, 316; AST: 198, 220, 221, 223, 327, 328, 333; BA: 50; CHE: 50, 198; CR:
192, 241, 243, 244; KDA: 78; KIR: 275; KR: 198, 212, 254; KYA: 122, 124; LEN: 161, 195, 198, 199, 212, 237; MOS: $66,70,159,160,164$; NVS: $301,308,310^{\mathrm{IG}}$; PRI: 218; SVE: 44, 50, 55, 198, 201, 212; TVE: 19, 76, 136, 179; VGG: 127, 198, 220, 223, 328.

Perichaena calongei Lado, D.Wrigley \& Estrada PRI: 218 .

Perichaena chrysosperma (Curr.) Lister - AL: 215; ALT: 84, 301, 308, 315, 317; AST: 198, 213, 220, 222, 223, 327, 328, 333; BA: 50, 198; CHE: 50, 198; CHU: 196, 198, 210, 212, 290; CR: 192, 241, 242, 243, 244; KAM: 198; KDA: 78, 192, 198; KHA: 198; KR: 198, 212, 254; KYA: 120, 122, 123, 124, 196, 198, 210, 211, 212, 290; LEN: 161, 182, 183, 195, 198, 199, 212, 237; MAG: 198; MOS: 7, 8, 9, 66, 70, 84, 160, 164, 198; NVS: 301, 308; ORE: 198, 202; PRI: 74, 218; ROS: 147, 198, 220, 223; SA: 288; SAM: 153; SVE: 46; TOM: 117, 132, 198; TVE: 19, 76, 165, 166; VGG: 110, 157, 198, 212, 220, 223, 284, 328, 332, 336, 338; VOR: 167 , 170, 192, 198; YAN: 50, 196, 198, 210, 212, 290.

Perichaena corticalis (Batsch) Rostaf. - ALT: 301, 308, 316, 317; AST: 198, 213, 220, 221, 222, 223, 327, 328, 333; BA: 50, 198; CHE: 50; CR: 243, 244; DA: 162; KDA: 78, 84, 192, 198; KHA: 198; KIR: 274, 275, 276; KK: $292^{\mathrm{IG}}$; KL: 198, 220, 222, 223, 328; KO: 50, 198; KR: 198, 212, 254; KRS: 5, 198; KYA: 101, 122, 124, 198, $292^{\mathrm{IG}}$; LEN: 101, 161, 180, 182, 183, 195, 198, 199, 212, 237, 323; LIP: 198, 252; MAG: 288; MOS: 1, 4, 6, 7, 8, 9 , 66, 70, 84, 159, 160, 164, 198; NVS: 301, 308; PRI: 74, 218; ROS: 198, 220, 223, 335; SA: 288; SAM: 153, 154; SMO: 101, 198; SVE: 42, 45, 50, 198, 201, 212, 236; TA: 101, 198; TOM: 132, 198; TVE: 6, 19, 76, 84, 136, 142, 165, 166, 179, 180, 182, 198, 204; TY: 304; VGG: 110 , 157, 198, 212, 220, 223, 281, 284, 285, 328, 332, 336, 338.

Perichaena depressa Lib. - AL: 134, 214, 215; ALT: 301, 308, 315, 316, 317; AST: 198, 213, 220, 221, 222, 223, 327, 328, 333; CHU: 196, 198, 210, 212, 290; CR: 243, 244; KDA: 78; KL: 198, 220, 222, 223, 328; KO: 50, 198; KRS: 185, 198; KYA: 121, 122, 124, 196, 198, 210 , 211, 212, 290; LEN: 161, 182, 183, 195, 198, 199, 212, 237; MOS: 6, 7, 8, 66, 70, 84, 164, 198; MUR: 65; NVS: 301, 308; ORE: 198, 202; PRI: 21, 218; SA: 288; SAM: 154; SMO: 99, 100, 101, 198; SVE: 40, 41, 42, 48, 50, 198, 201, 212; TVE: 136, 165, 166, 179; TY: 304; VGG: 110 , 157, 198, 220, 223, 281, 284, 328, 332, 336, 338; ZAB: 26. 
Perichaena heterospinispora Novozh., Zemly., Schnittler \& S.L.Stephenson - VGG: 224, 280, 282, $329,330,331,334$.

Perichaena liceoides Rostaf. - AL: 215, 318; ALT: 315, 317; AST: 198, 213, 220, 221, 222, 223, 327, 328, 333; CHU: 198, 212, 290; CR: 243, 244; KDA: 78; KL: 198, 220, 222, 223, 328; KYA: 121, 122, 124; MOS: 66, 70, 84; NVS: 318; TVE: 19, 76, 166; VGG: 110, 127, 198, 220, 223, 280, 284, 328, 332, 336, 337.

Perichaena luteola (Kowalski) Gilert - AL: 214, 215, 318; ALT: 318; AST: 213, 220, 223, 328, 329, 333; BU: 318; CR: 243, 244; NVS: 318; ZAB: 26.

Perichaena pedata (Lister \& G.Lister) G.Lister ex E.Jahn - AL: 134, 215 ${ }^{\mathrm{CF}}$; ALT: 301, 308, 315; CR: 174; MAG: 288; MOS: 66, 164; NVS: 301, 308; SA: 288; SVE: 236; VG G: 198, 220, 223, 328, 332, 338.

Perichaena polygonospora Novozh., Zemly., Schnittler \& S.L.Stephenson - AST: 224, 329, 330, 333; VG G: 224, 330, 334.

Perichaena quadrata T.Macbr. - AST: 213, 220, 223, 327, 328, 333; CR: 148; KL: 220, 223, 328; ORE: 202; VGG: 110, 127, 198, 212, 220, 223, 280, 284, 328, 332, 336, 337; ZAB: 26.

Perichaena syncarpon T.E.Brooks - SVE: 50, $201^{\mathrm{CF}}$.

Perichaena taimyriensis Novozh. \& Schnittler CHU: 198, 206; KYA: 198, 206, 212; ZAB: 26.

Perichaena vermicularis (Schwein.) Rostaf. - AL: 134, 214, 215; ALT: $301,308,310^{\mathrm{IG}}, 315,317$; AST: 198 , 213, 220, 221, 222, 223, 327, 328, 333; CHE: 50, 198; CR: 241, 243, 244; KDA: $78^{\text {CF }}$; KIR: 274, 276; KL: 198, 220, 222, 223, 328; KR: 198; KYA: 120, 121, 122, 123, 124 , 196, 198, 210, 211, 212, 290; LEN: 195, 198, 199, 212, 237; LIP: 198; MOS: 7, 15, 66, 238; NVS: 301, 308, 310 ORE: 198, 202; SA: 288; SAM: 153, 154; SVE: 46, 50, 198; TVE: 166; TY: 304; VGG: 110, 198, 212, 220, 223 , 280, 284, 285, 328, 332, 336, 338; ZAB: 26.

Physarella oblonga (Berk. \& M.A.Curtis) Morgan KDA: 163; PRI: 163.
Physarum albescens Ellis ex T.Macbr. - KAM: 219; KC: 34, 217, 255; LEN: 261; MUR: 34, 36, 258.

Physarum album (Bull.) Chevall. - AL: 3, 215; ALT: 209, 215, 226, 301, 308, 310 $0^{\mathrm{IG}}, 315,316,317$; AST: 198 , 220, 223, 327, 328, 333; BA: 50, 54, 198; CHE: 50, 198; CHU: 196, 198, 210, 212, 290; CR: 30, 101, 242, 243, 244; DA: 162; KAM: 251; KDA: 68, 198; KHA: 84, 198; KHM: 47, 52, 58; KIR: 14, 263, 264, 265, 267, 268, 275 , 276, 291; KLU: 101, 198; KO: 50, 198, 270; KOS: 101, 198; KR: 104, 198, 212, 254; KRS: 5, 185, 198, 209, 226; KYA: $10,120,122,123,124,129,196,198,209,210$, 211, 212, 226, 290; LEN: 89, 101, 180, 182, 183, 195, 198, 199, 209, 212, 226, 237, 286, 287, 323; LIP: 198, 252; MAG: 290; MOS: 1, 4, 6, 7, 8, 9, 11, 63, 64, 66, 70, 84, 101, 159, 160, 164, 179, 198, 278; MUR: 65, 67, 84, 104; NVS: 209, 226, 301, 308, 310 ${ }^{\mathrm{IG}}$; ORE: 50; PER: 50; PRI: 69, 74, 84, 198, 203, 218; PSK: 73, 140; ROS: 147, 198, 220, 223, 335; RYA: 84; SMO: 101, 198; SVE: 40, 41, 42, 43, 45, 49, 50, 51, 198, 201, 209, 212, 226, 236; TOM: $114,132,198$; TVE: $6,19,76,84,135,136,139$, 141, 142, 166, 179, 180, 182, 198, 204; TYU: $84,198^{\mathrm{IG}}$; VGG: $157,198,209,212,220,223,226,281,284,285$, 328, 332, 338; VLA: 173; VOR: 101, 167, 170, 198, 248; YAN: 50, 196, 198, 210, 212, 290.

Physarum alpestre Mitchel, S.W.Chapm. \& M.L. Farr - KAM: 219; KC: 34, 217, 255.

Physarum alpinum (Lister \& G.Lister) G.Lister AL: 3, 215; KAM: 219; KC: 34, 217; LEN: 180, 182, 183, 195, 197, 198, 212, 237, 282; MUR: 67; SVE: 212.

Physarum apiculosporum Härk. - AST: 220, 222, 223, 328, 333; IRK: 33; KYA: $121^{\mathrm{CF}}, 122^{\mathrm{CF}}, 124^{\mathrm{CF}}$; MOS: 66; VGG : 110, 157, 198, 220, 223, 328, 332.

Physarum aurantiacum Shuang L.Chen, Yu Li \& H.Z.Li - PRI: 218; SVE: 236.

Physarum auripigmentum G.W.Martin - PRI: 218.

Physarum auriscalpium Cooke - ALT: $310^{\mathrm{IG}}$; KIR: 274, 276; KR: 198, 212, 254; KYA: 122, 124; LEN: 195, 197, 198, 199, 212, 237, 282; MOS: 6, 7, 11, 64, 66, 70, 198; NVS: $301,307,308,310^{\mathrm{IG}}$; RYA: 84 ; SVE: 45,50 ; TVE: 165, 166. 
Physarum bethelii T.Macbr. ex G.Lister - ALT: 301, 308, 315; KYA: 122, 124; MOS: 164; PRI: 218; ROS: 147; SVE: 48, 50, 51, 198, 235, 236; TVE: 19.

Physarum bitectum G.Lister - ALT: 301, 308; AST: 198, 220, 223, 327, 328, 333; BA: 50; BU: 198; CHE: 198; IRK: 198; KR: 198, 212, 254; LEN: 182, 183, 195, 198, 212, 237; MOS: 66, 70, 164; NVS: 301, 308.

Physarum bivalve Pers. - AL: 215; ALT: 301, 308, 316, 317; CHU: 196, 198, 210, 212, 290; CR: 30, 242, 244; KDA: 90; KO: 50, 198; KR: 96, 104, 130, 198, 212, 254; KYA: 120, 122, 123, 124, 196, 198, 210, 211, 212 , 290; LEN: 101, 182, 183, 195, 198, 199, 212, 237, 323; LIP: 198, 252; MAG: 288, 290; MOS: 6, 7, 8, 66, 70, 84, 101, 198; MUR: 196, 198, 205, 210, 212, 290; ORE: 50, 198, 202; PRI: 22, 218; ROS: 198, 220, 223, 335; SA: 288; SMO: 101, 198; SVE: 48, 50, 198; TVE: 182; VGG : 198, 212, 220, 223, 284, 328, 337; VOR: 198.

Physarum braunianum de Bary - NVS: 301, 307, 308.

Physarum carneum G.Lister \& Sturgis - KR: 198, $212^{\mathrm{CF}}, 254^{\mathrm{CF}}$.

Physarum cinereum (Batsch) Pers. - AL: 3, 214, 215; ALT: 215, 301, 308, 315, 316, 317; AST: 213, 220, 222, 223, 327, 328, 333; BA: 50, 54; CHU: 196, 210, 212, 290; CR: 174, 192, 242, 243, 244; KDA: 90; KGD: 91; KHM: 52, 58; KL: 220, 222, 223, 328; KO: 50; KR: 198, 212, 254; KYA: 210, 211, 212, 290; LEN: 89, 101, 182, 183, 195, 199, 212, 237, 323; LIP: 252; MAG: 290; MOS: 6, 7, 8, 11 , 63, 66, 70, 84, 164, 278; MUR: 196, 205, 210, 212, 290; NVS: 301, 308; ORE: 202; PRI: 74, 218; SA: 288; SMO: 99 , 100, 101; SVE: 49, 50, 51, 236; TOM: 132; TVE: 6, 19, 76, 79, 84, 136, 204, 282, 296; VGG: 110, 212, 220, 223, 284, 285, 328, 332, 336, 338; VOR: 248, 249.

Physarum citrinum Schumach. - CR: 30, 243, 244; LIP: 198, 252; MOS: 4, 7, 66, 70; SVE: 40, 41, 50, 201, 212, 236; TVE: 79, 136, 198, 204, 282; VOR: 248.

Physarum compressum Alb. \& Schwein. - AL: 134, 215; ALT: 315, 316, 317; AST: 198, 213, 220, 222, 223, 327, 328, 333; BRY: 101 ${ }^{\text {OAU }}$; KDA: 78; KL: 328; KLU: 101 ${ }^{\text {OAU; KYA: }}$ 112, 113; LEN: 101, 180, 182, 183, 195, 198, 199, 212, 237, 323; LIP: 198, 252; MOS: 6, 7, 8, 9 , $66,70,94,101^{\text {OAU }}, 164,175,198,278$; NVS: 301,308 ;
PRI: 21; SMO: $101^{\text {OAU }}$, 198; SVE: 46, 48, 50, 198; TOM: 132, 198; TVE: $101^{\text {OAU }}, 136,166,198$; VGG: 110,198 , 220, 223, 328, 332, 338; VOR: 192, 198; ZAB: 26.

Physarum confertum T.Macbr. - AL: 215; KR: 198; LEN: 199; MOS: 6, 7, 63, 66, 70, 198, 278; SVE: 236; TVE: 84; VGG: 198.

Physarum conglomeratum (Fr.) Rostaf. - ALT: 302, 306; KYA: 122, 124; LEN: 183, 195, 198, 212, 323; MOS: 198; NVS: 301, 308; PRI: 218; SMO: 198; SVE: 50, 198, 201, 212; TVE: 136, 198, 204; VGG: 212, 220, 223, 328.

Physarum contextum (Pers.) Pers. - ALT: 301, 308; KO: 50, 198; KR: 198, 212, 254; LEN: 101, 180, 182, 183, 195, 198, 199, 212; Mos: 6, 7, 8, 66, 70, 84, 198, 278; MUR: 96, 104; NVS: 301, 308; ORE: 198, 202; PRI: 218; SAK: 198; SMO: 101, 198; SVE: 51; TOM: 132, 198; TVE: 19, 76, 136, 142, 198, 204; VLA: 173.

Physarum crateriforme Petch - ALT: 301, 307, 308, 317; NVS: 301, 307, 308; VOR: 170.

Physarum daamsii Nann.-Bremek. - KDA: 90.

Physarum decipiens M.A.Curtis - AL: 214, 215; ALT: $215,301,308,310^{\mathrm{IG}}, 315,316,317$; AST: 198,213 , 220, 221, 222, 223, 327, 328, 333; CR: 192, 244; KR: 198, 212, 254; KRS: 185; KYA: 120, 121, 122, 123, 124 , 198; LEN: 195, 198, 199, 212, 237; MOS: 6, 7, 66, 164 , 198, 278; NVS: 301, 308, 310 ${ }^{\mathrm{IG}}$; ORE: 198, 202; PRI: 218; SAM: 153; SMO: 101, 198; SVE: 50, 198, 201, 212; TOM: 198; TY: 304; VGG: 110, 157, 198, 220, 223, 284, 328, 332, 338; VOR: 167, 170, 192, 198.

Physarum dictyospermum Lister \& G.Lister $\operatorname{MOS}^{\mathrm{DR}}:$ 6, 7, 198.

Physarum diderma Rostaf. - AL: 198, 215; ALT: 301, 308, 317; AST: 223, 328; MOS: 8, 66, 84; NVS: 301, 308; PER: 50; ROS: 220, 223, 335; SVE: 49, 50, 198, 236; TVE: 19, 76; VGG: 198, 220, 223, 328.

Physarum didermoides (Pers.) Rostaf. - AL: 198, 215; ALT: 307, 317; AST: 198, 213, 220, 221, 222, 223, 328, 333; CHE: 50; KL: 220, 222, 223, 328; LEN: 182, 183, 195, 198, 212, 237; LIP: 198, 252; MOS: 6, 7, 8, 9, 66, 70, 84, 164, 198; NVS: 301, 308; PER: 50; SMO: 101, 198; SVE: 198; TVE: 19, 
76, 136; TY: 304; VGG: 110, 198, 220, 223, 328, 332, 338.

Physarum dispersum Y.Yamam. \& Nann.-Bremek. - NVS: 301, 308.

Physarum dubium Nann.-Bremek. \& Y.Yamam. SVE: 236.

Physarum famintzinii Rostaf. - KLU: 101, 198, 246; KO: 50, 198; TVE: 79, 80, 136, 198, 204, 282.

Physarum flagellatum (Alexeieff) Fiore-Donno, Kamono \& Caval.-Sm. - YAR: 59.

Physarum flavicomum Berk. - AL: 134; ALT: 215, 301, 308, 315, 316; BA: 50, 198; CHE: 50, 198; CR: 243, 244; KDA: 198; KHA: 198; KO: 50, 198; KR: 130; KYA: 122, 124; LEN: 182, 183, 195, 198, 212, 237; LIP: 198, 252; MOS: 6, 7, 11, 63, 66, 70, 84, 101, 198; PER: 50; PRI: 74, 203, 218; ROS: 147; SAM: 153, 154; STA: 198; SVE: 49, 50, 198, 236; TVE: 19, 76, 136, 204; VGG: 198, 212, 220, 223, 328, 330 .

Physarum flavidum (Peck) Peck - LEN: 182, 183, 195, 197, 198, 237, 282.

Physarum galbeum Wingate - MOS ${ }^{\mathrm{DR}}$ : 6, 198.

Physarum globuliferum (Bull.) Pers. - AL: 134, 215; ALT: 215, 301, 308, 310 ${ }^{\mathrm{IG}}, 316,317$; BA: 50, 54, 198; CHE: 50, 198; DA: $162^{\mathrm{CF}}$; KDA: 90; KLU: 8; KO: 50, 198; KR: 198, 212, 254; KYA: 120, 122, 123, 124; LEN: 182, 183, 195, 197, 198, 212, 237, 261, 282; LIP: 198, 252; MOS: 1, 6, 7, 8, 63, 66, 70, 198; NVS: 301, 308, 310 ${ }^{\mathrm{IG}}$; PRI: 69, 74, 218; ROS: 147; RYA: 84, 125, 321; SMO: 101, 198; SVE: 42, 50, 51, 198, 201, 212, 236; TOM: 132; TVE: 84, 136, 198, 204; TYU: 84; VOR: 248.

Physarum gyrosum Rostaf. - ALT: 301, 308, 317; AST: 328, 333; BU: 198; KHM: 47, 52, 58; KR: 198; LEN: 182, 183, 195, 198, 237; LIP: 198, 252; MOS: 7, 64, 66, 70; NVS: 301, 307, 308; PSK: 198; TY: 304; TYU: 198 ${ }^{\mathrm{IG}}$; VGG: 198, 220, 223, 328.

Physarum hongkongense Chao H.Chung - CR: 174.

Physarum javanicum Racib. - ALT: 301, 308; NVS: 301, 307, 308.
Physarum lakhanpalii Nann.-Bremek. \& Y.Yamam. - NVS: 320.

Physarum lateritium (Berk. \& Ravenel) Morgan AL: 215; ALT: $310^{\mathrm{IG}}$; MAG: 198; MOS: 8; NVS: 301, $308,310^{\mathrm{IG}}$.

Physarum lenticulare Nann.-Bremek. \& Y.Yamam. - NVS: 320.

Physarum leucophaeum Fr. \& Palmquist - AL: 3, 134, 198, 215; ALT: 209, 226, 301, 308, 315, 317; AST: 198, 213, 220, 222, 223, 327, 328, 333; ВА: 50, 54, 198; CR: 144, 243, 244; DA: $162^{\mathrm{CF}}$; IRK: 84; KDA: 68, 78; KHA: 84, 198; KL: 198, 220, 222, 223, 328; KO: 50, 198; KR: 198, 209, 212, 226, 254; KRS: 5, 185, 198, 226; KYA: 122, 124; LEN: 89, 180, 182, 183, 195, 198, 199, 209, 212 , 226, 237, 323; MAG: 290; MOS: 7, 8, 9, 63, 66, 70, 84, 164, 198; MUR: 84, 196, 198, 205, 210, 212, 290; NVS: 209, 226, 301, 308; PER: 50; PRI: 74, 203, 218; ROS 198, 220, 223; STA: 209, 226; SVE: 40, 41, 42, 49, 50, 51, 198, 201, 209, 212, 236; TVE: 19, 76, 84, 136, 165, 180, 182, 198, 204, 296; TYU: 84; VGG: 110, 157, 198, 209 , 212, 220, 223, 226, 284, 328, 332, 338; VLA: 173; VOR: 167, 170; YAN: 196, 198, 210, 212, 290; ZAB: 26.

Physarum leucopus Link - AL: 3, 191, 198, 215; ALT: 301, 302, 306, 308; BA: 50, 198; CHE: 50, 198; CR: 242, 244; DA: $162^{\mathrm{CF}}$; KDA: 68, 84; KHA: 84; KO: 50, 198; KR: 198, 212, 254; LEN: 101, 180, 182, 183, 195, 198, 212, 237 , 246, 323; LIP: 198, 252; MOS: 1, 7, 9, 11, 63, 64, 66, 70, 84, 164, 198; NVS: 301, 308; PER: 50; PRI: 74; SVE: 49, 50, 51 , 198, 201, 212, 236; TOM: 114, 132, 198; TVE: 19, 76, 84, 136, 141, 142, 166; TYU: 84; VGG: 198, 220, 223, 284, 328.

Physarum licheniforme (Schwein.) Lado - LEN: 246.

Physarum luteolum Peck - PRI: 218.

Physarum melleum (Berk. \& Broome) Massee KDA: 78, 84; PRI: 74, 218; VGG : 282, 338.

Physarum murinum Lister - MOS $^{\mathrm{DR}}$ : 6, 198, 278; RYA: 84.

Physarum mutabile (Rostaf.) G.Lister - ALT: $310^{\mathrm{IG}}$; MOS: $7,66,70,84,164$; NVS: $301,307,308,310^{\mathrm{IG}}$. 
Physarum newtonii T.Macbr. - PRI: 218.

Physarum nigripodum Nann.-Bremek. \& Y.Yamam. - NVS: 301, 307, 308.

Physarum nitens (Lister) Ing - AL: 134; MOS: 84.

Physarum nivale (Meyl.) Mar.Mey. \& Poulain KAM: 219; KC: 34, 209, 217, 226, 258.

Physarum notabile T.Macbr. - AL: 3, 198, 214 ${ }^{\mathrm{CF}}$, $215^{\mathrm{CF}}$; ALT: 209, 215, 226, 301, 308, 316, 317; AST: 198, 213, 220, 221, 222, 223, 327, 328; BA: 50, 54, 198; KDA: 78; KHA: 84; KHM: 52, 58; KIR: 274, 276; KL: 198, 220, 222, 223, 328; KO: 50, 198; KR: 198; KYA: 120, 122 , 123, 124, 198; LEN: 182, 183, 195, 198, 212, 237; MOS: $1,6,7,9,66,70,84,164,179,198$; NVS: 209, 226, 301, 308; ORE: 53, 198, 202; PER: 50; PSK: 209, 226; SAR: 198; SVE: 50, 51, 198, 201, 209, 212, 226, 236; TVE: 19, 76, 136, 142, 179; TYU: 84; VGG: 110, 157, 198, 212, 220, 223, 284, 328, 332, 338.

Physarum nucleatum Rex - LEN: 180, 181, 198; TVE: 19; VOR: 167.

Physarum nudum T.Macbr. - ALT: 315, 317; AST: 198, 220, 223, 327, 328, 333; KYA: 196, 198, 210, $211^{\mathrm{CF}}$, $212^{\mathrm{CF}}, 290^{\mathrm{CF}}$; LEN: 195, 198, $212^{\mathrm{CF}}, 237$; MOS: 66,70 , 278; SVE: 50, 198.

Physarum oblatum T.Macbr. - CHU: 196, 198, 210, 212, 290; CR: 192, 244; KHA: 198; KR: 198, 212, 254; KYA: 198, 210, 211, 212, 290; LEN: 180, 181, 182, 183, 195, 198, 212, 237; MAG: 290; PRI: 218; SVE: 198, 201, 212; TVE: 19, 79, 80, 136, 182, 282; YAN: 50, 196, 198, 212, 290.

Physarum ovisporum G.Lister - VGG: 110, 198, 220, 223, 328, 332, 337.

Physarum penetrale Rex - KYA: 122, 124; PRI: 218; TVE: 76, 84; VLA: 172.

Physarum pezizoideum (Jungh.) Pavill. \& Lagarde - KIR: 268, 269; LEN: 182, 183, 195, 198, 212, 237; MOS: 6, 9, 66, 70, 101, 164, 198; ROS: 335; STA: 198; VGG: 198, 212, 220, 223, 282, 284, 285, 328, 329, 338.

Physarum polycephalum Schwein. - KR: 130 MOS: 6, $7^{\mathrm{CF}}, 66,70$; TVE: 6, 136, 204.
Physarum pseudonotabile Novozh., Schnittler \& Okun - AL: 209; ALT: 225, 226, 315; AST: 209, 225, 226, 333; CR: 257; KC: 225, 226; KL: 209; VGG: 27, 209, 225, 226, 280, 337.

Physarum psittacinum Ditmar - AL: 3, 191, 198, 215, 300; ALT: 301, 307, 308; BA: 54; BRY: 16, $101^{\mathrm{OAU}}$; BU: 198; CHE: 50, 198; KIR: 268; KO: 50, 198; KYA: 122, 124; LEN: 323; MOS: 1, 4, 6, 7, 9, 64, 66, 70, 84, 198, 278; NVS: 301, 307, 308; PSK: 73; SVE: 49, 50, 51, 198, 201, 212, 236; TVE: 6, 19, 76, 84, 136, 141, 198, 204.

Physarum pulcherrimum Berk. \& Ravenel - AL: 215; LEN: 182, 183, 195, 198, 212, 237; PRI: 218; RYA: 125, 321; TVE: 19, 76, 141.

Physarum pulcherripes Peck - CHE: 50; KO: 50; PER: 50; SVE: 50, 198, 201, 212, 236.

Physarum pusillum (Berk. \& M.A.Curtis) G.Lister - AST: 198, 209, 220, 222, 223, 226, 327, 328, 333; BA: 50, 198; CR: 148; KL: 209, 220, 222, 223, 226, 328; KYA: 122 , 124; MOS: $6,7,66,70,84,164,198$; ORE: 198, 202; TVE: 165, 166.

Physarum rubiginosum Fr. \& Palmquist - BA: 198; KO: 50, 198; KYA: 122, 124; LEN: 101, 183, 195, 198, 212, 237, 246, 323; MOS: 6, 198, 278; SMO: 101, 198.

Physarum schroeteri Rostaf. - LEN: 182, 183, 195, 237; NVS: 309, 311; PRI: 74, 203; ROS: 335.

Physarum serpula Morgan - BA: 50; KDA: 78; LEN: 182, 183, 195, 212, 237; MOS: 6, 7, 8, 66, 70, 84, 278; RYA: 125, 321; SVE: 50, 201 ${ }^{\mathrm{CF}}$, 212; TVE: 136, 142, 165, 166.

Physarum sessile Brândză - AST: 220, 222, 223, 328, 333; PRI: 218.

Physarum spectabile Nann.-Bremek., Lado \& G.Moreno - MOS: 84; TVE: 19, 20, 75, 76; TYU: 84.

Physarum stellatum (Massee) G.W.Martin - MOS $^{\mathrm{DR}}$ : 6, 198; PRI: 218; VOR: 167, 170.

Physarum straminipes Lister - ALT: 317; AST: 198, 220, 223, 327, 328, 333; CHE: 50, 198; MOS: 198; SVE: 
42, 50, 198, 201, 212, 236; VGG: 110, 157, 198, 212, 220, 223, 284, 328, 332.

Physarum sulphureum Alb. \& Schwein. - CHE: 198;

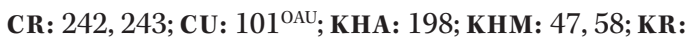
198; KYA: 122, 124; LEN: 101, 183, 195, 198, 212, 237, 246; LIP: 198, 252; ME: 101 ${ }^{\text {OAU }}$; MOS: 9, 15, 198, 238; NVS: 301, 307, 308; PRI: 74, 203; SAM: $101^{\text {OAU }}$;VE: 198, 236; TA: $101^{\text {OAU }}, 198,262$; TOM: 132, 198; TVE: 76; TYU: $198^{\mathrm{IG}}$; ULY: $101^{\mathrm{OAU}}$.

Physarum tenerum Rex - KDA: 198; KRS: 5, 198; LEN: 181, 182, 183, 195, 198, 212, 237; MOS: 6, 7, 9, 66, 70, 198; PRI: 74, 218; RYA: 125, 321; SVE: 198, 201; TVE: 19, 136, 180, 198, 204.

Physarum umbiliciferum Y.Yamam. \& Nann.Bremek - PRI: 218.

Physarum vernum Sommerf. - AST: 198, 220, 222, 223, 327, 328, 333; KAM: 219; KC: 34, 216, 217, 255; KDA: 78; KYA: 122, 124; LEN: 34, 35, 181, 182, 183, 195, 198, 212, 237; LIP: 198, 252; MOS: 7, 8, 9, 11, 15, 66, 70, 84, 164, 198; NVS: 301, 308; PSK: 198; SAM ${ }^{\mathrm{DR}}$ : 154, 156; SVE: 42, 44 ${ }^{\mathrm{CF}}$, 46, 49, 50, 198, 201, 212; TVE: 19, 136, 165, 180, 198; VGG: 110, 157, 198, 220, 223, 284, 285, 328, 332.

Physarum virescens Ditmar - KR: 198, 212, 254; KYA: 122, 124; LEN: 101, 180, 182, 183, 195, 198, 199 , 212, 237, 246, 323; Mos: 6, 7, 8, 66, 70, 84, 198, 278; MUR: 196, 205, 210, 212, 290; PRI: 218; PSK: 198; TOM: 198; TVE: 19, 76, 136, 179, 198; VGG: 198, 212, 220, 223, 328.

Physarum viride (Bull.) Pers. - AL: 134, 191, 215; ALT: 215, 301, 308, 316; BA: 50, 54, 198; CHE: 50; CHU: 196, 198, 210, 212, 290; CR: 30, 244; KDA: 90; KHM: 47, 52, 58, 196; KIR: 268, 291; KO: 50, 198; KR: 198, 212, 254; KRS: 5, 185, 198; KYA: 122, 124, 196, 198, 210, 211, 212, 290; LEN: 89, 101, 180, 182, 183, 195, 198, 199, 212, 237; LIP: 198, 252; MOS: 1, 4, 6, 7 , 8, 9, 11, 63, 64, 66, 70, 84, 164, 198, 278; MUR: 196, 198, 205, 210, 212, 290; NVS: 301, 308; ORE: 198, 202; PER: 50; PRI: 21, 69, 74, 84, 203, 218; PSK: 73; ROS: 147, 198, 220, 223; RYA: 84, 125, 321; SAM: 153; SMO: 101 , 198; SVE: 40, 41, 50, 51, 198, 201, 212, 236; TOM: 132; TVE: 19, 76, 84, 136, 141, 142, 182; TYU: $84,198^{\mathrm{IG}}$;
VGG: $157,198,212,220,223,284,328,338$; VLA: 173; VOR: 167, 170, 171, 248; YAN: 198, 210, 212, 290.

Protophysarum phloiogenum M.Blackw. \& Alexop. - ALT: 315, 316; AST: 198, 213, 220, 222, 223, 328; VOR: 25 .

Prototrichia metallica (Berk.) Massee - AL: 191, 215; CHU: 189, 196, 198, 210, 212, 290; KHM: 52, 58; KO: 50, 198; KYA: 196, 198, 210, 211, 212, 290; LEN: 101, 183, 195, 198, 212, 237; MAG: 198; PER: 50; PRI: 218; SVE: 50, 198, 201, 212; TVE: 79, 80, 136, 204, 282; YAN: 210.

Reticularia intermedia Nann.-Bremek. - DA: 162; IRK: 84; KHM: 47, 58; KIR: 276, 277; KO: 50, 198; KR: 198; KRS: 5, 198; KYA: 122, 124; LEN: 182, 183, 195, 198, 212, 237; MOS: 66, 70, 84; MUR: 84; PRI: 74; PSK: 73; ROS: 335; SVE: 40, 41, 50, 51, 198, 201; TVE: 19 , 136, 198, 204; TYU: 198 ${ }^{\mathrm{IG}}$; VGG: 198, 220, 223, 284 , $285,328$.

Reticularia jurana Meyl. - CR: 174, 243; KR: 149, 212, 254; KYA: 196, 210, 211, 212, 290; LEN: 195, 197, 212, 237; NVS: 301, 307, 308; SVE: 201, 212; VG G: 212.

Reticularia liceoides (Lister) Nann.-Bremek. KHM: 52, 58 .

Reticularia lycoperdon Bull. - AL: 3, 134, 215, 300; ALT: 317; BRY: 101 ${ }^{\text {OAU }}, 126$; CHU: 189, 196, 198, 210, 212, 290; CR: 84, 244; DA: 162; IRK: 84; KDA: 68; KHM: 47, 52, 58; KIR: 268; KLU: 101, 198; KO: 50, 198; KR: 104, 130, 198, 212, 254; KRS: 5, 101, 185, 198; KYA: 10, 101, 129, 198, 210; LEN: 89, 96, 101, 182, 183, 195, 198, 212, 237, 323; LIP: 198, 252; MAG: 198; MOS: 4, 6, 7, 8, 9, 11, 66, 70, 84, 164, 198, 278; MUR: 65, 84, 96, 104; NIZ: 101, 176, 198; NVS: 198, 233, 301, 308; PER: 50; PRI: 22, 74, 177; PSK: 73, 101, 140, 198; ROS: 147; RYA: 125, 321; SAM: 154; SE: 198; SMO: 101, 198; STA: 57, 97, 98, 101, 198; SVE: 40, 41, 50, 198, 201, 212, 236; TA: 101, 198, 262; TOM: 132, 198; TVE: 6, 76, 84, 135, 136, 139, 142, 166, 179, 182, 198, 204; TYU: 101, 198 ${ }^{\mathrm{IG}}$; VGG: 198, 212, 220, 223, 328, 338; VLA: 173; VLG: 101, 198; VOR: 171, 248; ZAB: 198.

Reticularia olivacea (Ehrenb.) Fr. - CHU: 189, 196, 198, 210, 212, 290; KR: 130; MAG: 198; MO' ${ }^{\text {DR }}$ : 1: 6; SMO: 101, 198; TOM: 115. 
Reticularia splendens Morgan - AL: 134, 215; ALT: 301, 308; BA: 50; BU: 198; CR: 144, 244; DA: 162; KAM: 251; KHA: 84; KHM: 47, 58; KO: 50, 198; KR: 84; KYA: $112,113,120,122,123,124,198$; LEN: 198, 199, 237; MOS: 6, 7, 66, 70, 84, 164, 198; MUR: 65, 84; NVS: 149, 301, 308; PRI: 74, 203, 218; PSK: 73; ROS: 147, 149, 198, 220, 223; SVE: 50, 51, 149, 198; TOM: 114, 115, 117, 132, 198; TVE: 136, 204; TYU: $198^{\mathrm{IG}}$; VGG: 157, 198, 220, 223, 328, 330; VLA: 173; VOR: 167, 170.

Siphoptychium casparyi Rostaf. - AST: 198, 220, 223, 328; KO: 198; SVE: 51, 198; VGG: 281, 329.

Stemonaria gracilis Nann.-Bremek. \& Y.Yamam. CR: 145, 148; KDA: $78^{\mathrm{CF}}$; MOS: $66,70$.

Stemonaria irregularis (Rex) Nann.-Bremek., R.Sharma \& Y.Yamam. - AL: 133, 134, 215; ALT: 215, 301, 308, 316; BA: 50, 198; IRK: 84; KHA: 198; KO: 50 , 198; LIP: 198, 252; MOS: 6, 7, 9, 66, 70, 84, 198, 278; NVS: 301, 308; PER: 50; PRI: 74, 84, 203, 218; SVE: 45, 50, 198, 201, 212, 236; TOM: 132, 198; TVE: 84; TY: 304; VGG: 88, 198, 220, 223, 282, 328, 338; VLA: 172.

Stemonaria longa (Peck) Nann.-Bremek., R.Sharma \& Y.Yamam. - AST: 198, 213, 220, 223, 327, 328, 333; CR: 30, 101, 145; KO: 50, 198; LEN: 101, 183, 195, 197, 212, 237; MOS: 6, 101, 198; ROS: 335; RYA: 125, 321; SMO: 101; SVE: 51; VGG: 88, 198, 328, 338.

Stemonaria nannengae (T.N.Lakh. \& K.G.Mukerji) Nann.-Bremek., R.Sharma \& Y.Yamam. - ALT: $310^{\mathrm{IG}}$; NVS: $301,307,308,310^{\mathrm{IG}}$.

Stemonitis axifera (Bull.) T.Macbr. - AL: 3, 134, 191, 198, 215; ALT: 215, 301, 302, 306, 308, 316, 317; AST: 220, 223, 328; BA: 50, 54, 198; BRY: $101^{\mathrm{OAU}}, 111$; BU: 198; CE: 57, 97, 98, 101, 198; CHE: 50, 198; CHU: 196, 198, 210, 212, 290; CR: 30, 84, 101, 145, 242, 243, 244; DA: 162; IRK: 84; KAM: 251; KC: 57, 97, 98, 101, 198; KDA: 68, 84, 192, 198; KGD: 91; KHA: 84; KHM: 47, 52, 58, 196; KIR: 263, 264, 265, 268, 269; KL: 220, 222 , 223, 328; KLU: 101 ${ }^{\mathrm{OAU}}$; KO: 50, 198; KR: 84, 198, 212 , 254; KRS: 5, 185, 198; KYA: 10, 112, 120, 122, 123, 124 , 129, 196, 198, 210, 211, 212, 290; LEN: 89, 101, 180, 182, 183, 195, 198, 199, 212, 237, 286 ${ }^{\mathrm{DS}}, 287^{\mathrm{DS}}$; LIP: 198, 252; MAG: 198, 290; MOS: 1, 2, 4, 6, 7, 8, 9, 11, 63, 64, 66, 70, 84, 92, 101, 164, 175, 198, 278; MUR: 67, 84 ,
106, 196, 198, 205, 210, 212, 290; NVS: 198, 233, 301, 308; ORE: 198, 202; ORL: 168; PER: 50; PRI: 21, 22, 69, 74, 84, 218; PSK: 73, 101, 198; ROS: 335; RYA: 84, 125, 321; SAM: 153, 156; SMO: 99, 100, 101 ${ }^{\mathrm{OAU}}, 198$; STA: 198; SVE: 40, 41, 50, 51, 198, 201, 212, 234, 236; TA: 101, 198, 262; TOM: 132, 198; TVE: 6, 19, 76, 84, 101, 135, 136, 139, 141, 166, 179, 180, 182, 198, 204, 296; TYU: 84, $198^{\mathrm{IG}}$; VGG: 88, 157, 198, 212, 220, 223, 284, 285, 328, 336, 338; VLA: 173; VOR: 167, 171, 248; YAN: 50, 196, 198, 210, 212, 290; ZAB: 101, 105, 198.

Stemonitis capillitionodosa G.Moreno, D.W.Mitch., C.Rojas \& S.L.Stephenson - MUR: 319.

Stemonitis flavogenita E.Jahn - AL: 134; ALT: 317; CHE: 50; CR: 145, 174, 243, 244; KO: 50, 198; KR: 198; KYA: 122, 124; LIP: 198, 252; MOS: 4, 6, 7, 66, 70, 198, 278; PER: 50; PRI: 21, 22, 74; ROS: 147; RYA: 84; SVE: 198; TVE: 6, 84, 136, 179, 198, 204; VGG: 88, 284 , $285,338$.

Stemonitis fusca Roth - AL: 3, 134, 191, 215; ALT: 215, 299, 301, 302, 306, 308, 316, 317; ARK: 143; AST: 213, 220, 222, 223, 327, 328, 333; BA: 50; BRY: 16, $101^{\mathrm{OAU}}$; CHU: 196, 210, 212, 290; CR: 145, 242, 243, 244; DA: 162; KAM: 297; KC: 57, 97, 98; KDA: 78, 90, 192; КHM: 52, 58, 196; KIR: 14, 263, 264, 265, 266, 268, 269, 271, 275, 291; KK: 250 ${ }^{\mathrm{IG}}, 292^{\mathrm{IG}}$; KLU: 101; KO: 50; KOS: 101; KR: 104, 130, 143, 212, 254; KRS: 5, 185; KYA: 101, 112, 122, 124, 250 ${ }^{\mathrm{IG}}, 292^{\mathrm{IG}}$; LEN: 89, 96, 101, 183, 195, 199, 212, 237; LIP: 252; MOS: 1, 4, 6, 7, 8, 9, 11, 18, 63, 64, 66, 70, 84, 94, 101, 164, 175, 179, 278; MUR: 84, 104; NIZ: 101, 176; NVS: 233, 301, 308; ORL: 168; PER: 50; PRI: 21 , 22, 74, 84, 177, 218; PSK: 73, 101; ROS: 147, 335; RYA: 125, 321; SMO: 99, 100, 101; STA: 57, 97, 98, 101; SVE: 40, 41, 42, 43, 49, 50, 51, 201, 212, 236; TA: 101, 262; TOM: 114, 132, 233; TVE: 6, 19, 76, 84, 136, 141, 179, 204; TY: 304; TYU: 84; VGG: 88, 110, 157, 212, 220, 223, 284, 285, 328, 332, 336, 337, 338; VLG: 101; VOR: 167, 170, 171, 248, 249; YAN: 210, 212, 290; YAR: 101.

Stemonitis herbatica Peck - ALT: 215, 301, 308, 316; CR: 30, 145, 174, 242, 243, 244; KYA: 122, 124; LIP: 198, 252; MOS: 4, 6, 7, 66, 70, 164, 198; PRI: 218; SVE: 50, 198, 212; TOM: 117.

Stemonitis inconspicua Nann.-Bremek. - ALT: 301, 308. 
Stemonitis marjana Y.Yamam. - PRI: 74; SVE: 236.

Stemonitis pallida Wingate - AL: 3, 215; AST: 198, 220, 223, 327, 328, 333; CHE: 50, 198; CR: 30, 145, 244; KDA: 68; KHM: 47, 52, 58; KLU: 8; KO: 50, 198; KYA: 120 , 122, 123, 124; LEN: 180, 181, 182, 183, 195, 198, 199, 212, 237; MOS: 1, 7, 8, 9, 63, 66, 70, 164, 198; MUR: 65; PRI: 74; PSK: 73; SVE: 40, 41, 42, 50, 51, 198, 201, 236; TVE: $19,84,136,179,180,182,198,204$; TYU: $198^{\mathrm{IG}}$; VGG: $88,157,198,220,223,284,328$, 338; VOR: 167.

Stemonitis pseudoflavogenita A. Vlasenko et Novozh. - KDA: 319; NVS: 319.

Stemonitis splendens Rostaf. - AL: 3, 215; ALT: 215, 299, 302, 306, 316, 317; AST: 84, 220, 223, 327, 328, 333; CHE: 50, 198; CR: 30, 145, 244; KDA: 68, 90, 192; KIR: 265; KR: 84; KRS: 5; KYA: 10, 120, 122, 123, 124, 129; LEN: 180, 182, 183, 195, 197, 212, 237, 246, 282; LIP: 252; MOS: 4, 6, 7, 8, 9, 64, 66, 70, 84, 164, 278; MUR: 84; NVS: 301, 308; PRI: 74, 84, 218; PSK: 73, 140; ROS: 147; RYA: 84,125 , 321; SAM: 153, 156; SMO: 101; SVE: 50; TOM: 115, 132; TVE: 19, 76, 84, 135, 136, 139, 142, 166, 179, 182, 204; TYU: 84; VGG: 88, 157, 198, 212, 220, 223 , 281, 284, 285, 328, 329, 336, 338; VOR: 171, 248.

Stemonitis uvifera T.Macbr. - PRI: 84.

Stemonitis virginiensis Rex - BU: 198; CR: 144, 145; KHM: 47, 58; KO: 50, 198; KR: 198, 212, 254; LEN: 195, 198, 199, 212, 237; Mos: 6, 7, 63, 66, 70, 198, 278; MUR: 196, 205, 210, 212, 290; SVE: 236; TVE: 19; TY: 304; TYU: $198^{\mathrm{IG}}$.

Stemonitopsis aequalis (Peck) Y.Yamam. - AL: 198, 215; BA: 54; CR: 148; KDA: 90; KO: 50, 198; MOS: 66, 70; PRI: 74, 218; PSK: 73; SA: 288; SVE: 236; TVE: 19, 76.

Stemonitopsis amoena (Nann.-Bremek.) Nann.Bremek. - AL: 215; ALT: 215; BA: 50, 198; CHE: 198; CR: 145; KR: 198; NVS: 301, 308; PRI: 218; PSK: 73.

Stemonitopsis gracilis (G.Lister) Nann.-Bremek. AL: 215; ALT: 215, 307, 316; MOS: 66, 70; NVS: 301, 307, 308; PRI: 74; SVE: 51; TVE: 76.

Stemonitopsis hyperopta (Meyl.) Nann.-Bremek. AL: 191, 198, 215; AST: 198, 220, 223, 327, 328, 333;
BA: 54; CR: 145, 244; KDA: 78; KL: 198; KO: 50, 198; KR: 84, 198, 212, 254; KYA: 122, 124, 129; MOS: 1, 4, 6, 7, 9, 63, 66, 70, 84, 164, 198, 278; MUR: 84, 196, 205, 210, 212, 290; PRI: 74, 218; PSK: 73; RYA: 84; SVE: 40, 41, 43, 45, 50, 198, 201, 212, 236; TVE: 19, 76, 84, 136, 198, 204; TYU: 84; VGG: 88, 110, 157, 198, 212, 220 , 223, 284, 328, 332, 338.

Stemonitopsis microspora (Lister) Nann.-Bremek. - LEN: 180; SVE: 50, 198, 201, 212.

Stemonitopsis reticulata (H.C.Gilbert) Nann.Bremek. \& Y.Yamam. - KDA: 78; KR: 254; MOS: 7, 66, 84; TY: 304.

Stemonitopsis subcaespitosa (Peck) Nann.Bremek. - BA: 50; CHE: 198; CR: 241; KYA: 196, 198, 210, 211, 212, 290.

Stemonitopsis typhina (F.H.Wigg.) Nann.-Bremek. AL: 3, 134, 198, 215; ALT: 215, 301, 308, 316; BA: 50, 198; CHE: 50, 198; CHU: 196, 198, 210, 212, 290; CR: 243, 244; DA: 162; KAM: 198, 251; KDA: 68; KHA: 198; KHM: 47, 58; KIR: 263, 264, 265, 266, 268, 271, 275; KO: 50, 198; KR: 130, 198, 212, 254; KRS: 185, 198; KYA: 10, 120, 122, 123 , 124, 129, 198; LEN: 101, 180, 182, 183, 195, 198, 199, 212 , 237; LIP: 198, 252; MAG: 198, 290; MOS: 1, 4, 6, 7, 8, 9, 11, $63,64,66,70,84,101^{\text {OAU }}, 158,164,198,278,289$; MUR:

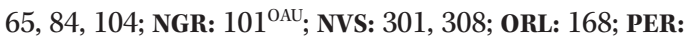
50; PRI: 21, 69, 74, 84, 218; PSK: 73; RYA: 125, 321; SMO: 101, 198; SVE: 40, 41, 49, 50, 51, 198, 201, 212, 236; TOM: 132, 198; TVE: $19,76,84,101^{\text {OAU }}, 136,141,180,182,198$, 204; TYU: 84, $198^{\mathrm{IG}}$; VGG: $88,198,212,220,223,284,328$, 338; VLA: 173; VOR: 170, 248; YAR: $101^{\text {OAU }}$.

Symphytocarpus amaurochaetoides Nann.Bremek. - AL: 215; ALT: 301, 308, 317; CR: 174, 243, 244; KHM: $52^{\mathrm{CF}}, 58^{\mathrm{CF}}$; KYA: $120,122,124,198$; MOS: 66, 70, 84; PRI: 218; TVE: 136.

Symphytocarpus confluens (Cooke \& Ellis) Ing \& Nann.-Bremek. - AL: 3, 134, 198, 215; KDA: 78; KR: 198, 212, 254; LEN: 182, 183, 195, 199, 212, 237; MOS: 6, 7, 8, 66, 70, 198, 278; TVE: 136, 204; VGG : 88, 280, 338; VOR: 248.

Symphytocarpus flaccidus (Lister) Ing \& Nann.Bremek. - AL: 198, 215; ALT: 301, 307, 308, 310 
316; AST: 198, 213, 220, 222, 223; BA: 50, 198; CHE: 50, 198; KHA: 198; КНM: 47, 58; KR: 84, 198, 212, 254 ; KYA: 122, 124; MAG: 198; MOS: 198; MUR: 84; NVS: 310 ${ }^{\mathrm{IG}}$; PSK: 73; SVE: 198, 201, 212; TVE: 84, 136, 179, 198, 204; TYU: $198^{\mathrm{IG}}$; VGG: 157, 198, 220, 223, 328.

Symphytocarpus herbaticus Ing - BA: 50, 198; KHM: 47, 58; TYU: $198^{\mathrm{IG}}$.

Symphytocarpus impexus Ing \& Nann.-Bremek. CHE: 50; CR: 243, 244; KR: 103; MOS: 84; TVE: 79, 80, $84,136,142,282$.

Symphytocarpus trechispora (Berk. ex Torrend) Nann.-Bremek. - KR: 198.

Thecotubifera dictyoderma (Nann.-Bremek. \& Loer.) Leontyev, Schnittler, S.L.Stephenson \& Novozh. - KHM: 52, 58; PRI: 218; SVE: 51, 236.

Trichia affinis de Bary - AL: 134; BA: 54; KDA: 68; KYA: 10, 198; LEN: 101, 198; MOS: 7, 278; MUR: 65 ${ }^{\mathrm{CF}}$; ROS: 147; TA: 101, 198, 262; TOM: 132, 198; TVE: 19, 141.

Trichia alpina (R.E.Fr.) Meyl. - AL: 134; KAM: 219; KC: $34,217,255$; KO: 50, 198; LEN: 182, 183, 195, 197, 198, 212, 237, 282; MUR: 34, 36, 196, 198, 205, 210, 212, 290; SVE: 236; ZAB: 26.

Trichia botrytis (J.F.Gmel.) Pers. - AL: 3, 84, 191, 198, 215; ALT: 215, 301, 308; BA: 50, 54, 198; BU: 198; CHE: 50; CHU: 196, 198, 210, 212, 290; CR: 244; IRK: 198; KDA: 198; KHA: 198; KHM: 47, 52, 58; KIR: 108, 265; KLU: 84; KO: 50, 198; KR: 130, 198, 212, 254; KYA: $120,122,123,124,196,198,210,211,212,290$; LEN: 101, 182, 183, 195, 198, 212, 237; LIP: 198, 252; MAG: 288; MOS: 4, 6, 7, 8, 9, 64, 66, 70, 84, 164, 198, 278; MUR: 65, 67, 84, 196, 198, 205, 210, 212, 290; NVS: 301, 308; PER: 50; PRI: 21, 69, 74, 203, 218; PSK: 140; SA: 288; SMO: 101, 198; SVE: 42, 49, 50, 51, 198, 201, 212, 236; TA: 101, 198, 262; TVE: 6, 19, 76, 136, 139, 141, 142, 166, 179, 180, 182, 198, 204; TYU: $198^{\mathrm{IG}}$; VGG: 27; VOR: 101, 171, 248; YAN: 50, 196, 198, 210 , $212,290$.

Trichia brevicapillata Sizova, Titova \& Darakov MOS: $6,7,66,70,278,279$.
Trichia brunnea J.J.Cox - ALT: 318; AST: 220, 223; VGG: $220,223$.

Trichia contorta (Ditmar) Rostaf. - AL: 134, 215; ALT: 301, 308, 317; AST: 220, 223, 327, 328, 329, 333; BA: 50, 198; CR: 84, 148, 243, 244; DA: 162; KDA: 78, 198; KHM: 47, 58; KO: 50, 198; KR: 198, 212, 254; KRS: 12; KYA: 10, 121, 122, 124; LEN: 180, 182, 183, 195, 198, 212, 237; LIP: 198, 252; MAG: 288; MOS: 6, 7, 8, 66, 70, 84, 164, 198, 278; MUR: 65, 67, 196, 198, 205, 210, 212, 290; NVS: 301, 308; PER: 50; PRI: 218; SAM: 155; SMO: 101, 198; SVE: 48, 49, 50, 51, 198, 201, 212 , 236; TA: 101, 198, 262; TOM: 115, 132, 198; TVE: 19, 76, 136, 165, 166, 180, 182, 198; TYU: $198^{\mathrm{IG}}$; VGG : 157 , 198, 212, 220, 223, 284, 328, 338.

Trichia crateriformis G.W.Martin - KDA: $78^{\mathrm{CF}}$; MOS $^{\text {DR }}:$ 6, 198, 278.

Trichia decipiens (Pers.) T.Macbr. - AL: 3, 191, 215; ALT: 215, 301, 302, 306, 308, 316; BA: 50, 54, 198; BRY: 16, $101^{\mathrm{OAU}}$; CHE: 50, 198; CHU: 189, 196, 198, 210, 212 , 290; CR: 144, 241, 243, 244; DA: 162; IRK: 84; KAM: 198, 251, 297; KDA: 68; КНА: 198; КНM: 47, 52, 58; KIR: 107, 263, 264, 265, 266, 268, 269, 272, 273, 275, 276, 277, 291; KLU: $101^{\mathrm{OAU}}$; KO: 50, 198; KOS: 101, 198; KR: 104, 198, 212, 254; KRS: 198; KYA: 10, 120, 122, 123, 124, 196, 198, 210, 211, 212, 290; LEN: 89, 96, 101, 180, 182, 183, 195, 198, 199, 212, 237, 323; MAG: 290; MOS: 1, 4, 6, 7, $8,9,11,18,63,64,66,70,84,94,101,164,175,179,198$, 278; MUR: 65, 104, 196, 198, 205, 210, 212, 290; NVS: 301, 308; PER: 50; PRI: 74, 203, 218; PSK: 73, 101, 140, 198; SAK: 198; SMO: $101^{\mathrm{OAU}}$, 198; STA: 198; SVE: 40, 41, 49, 50, 51, 198, 201, 212, 236; TA: 101, 198, 262; ТОМ: 132, 198; TVE: 19, 76, 84, $101^{\mathrm{OAU}}, 136,141,179,180,182$, 198, 204; TYU: 84, $198^{\mathrm{IG}}$; VGG: 198, 212, 220, 223, 284, 285, 328, 330, 338; VLA: 173; VOR: 171, 248.

Trichia elaterensis (Mulleavy) Lado - AST: 198, 213, 328, 329, 330, 333; VGG : 198, 328, 330.

Trichia erecta Rex - AL: 215; ALT: 215; BA: 54; KHM: 47, 58; KO: 50, 198; MOS: 8, 66; MUR: 196, 198, 205, 210, 212, 290; PRI: 218; SVE: 236; TVE: 19, 76, 141; TYU: $198^{\mathrm{IG}}$.

Trichia favoginea (Batsch) Pers. - AL: 3, 191, 214, 215; ALT: 215, 301, 302, 306, 308, 316; BA: 50, 54, 198; 
BRY: 16, 101 ${ }^{\text {OAU, }}$ CHE: 50, 198; CR: 30, 84, 243, 244; DA: 162; KAM: 251; KDA: 198; KHA: 198; KHM: 47, 58; KIR: 263, 264, 265, 268, 269, 272, 275, 291; KK: $295^{\mathrm{IG}}$; KLU: 101, 198; KO: 50, 198; KR: 198, 212, 254; KRS: 5, 101, 185, 198; KYA: $10,101,120,122,123,124,198,295^{\mathrm{IG}}$; LEN: 89, 101, 180, 182, 183, 195, 198, 199, 212, 237, $286^{\text {DS }}, 287^{\text {DS }}, 323$; MOS: $1,4,6,7,9,11,18,63,64,66,70$, 84, 101, 164, 175, 198, 278; MUR: 65, 67, 196, 205, 210 , 212, 290; NGR: 101, 198; NVS: 301, 308; PER: 50; PRI: 22, 74, 203, 218; PSK: 73, 101, 198; RYA: 84, 125, 321; SAR: 101; SMO: 101, 198; STA: 198; SVE: 40, 41, 49, 50, 51, 198, 201, 212, 236; TA: 101, 198, 262; TOM: 114,132 , 198; TVE: $6,19,76,84,101^{\text {OAU }}, 136,141,179,180,182$, 198, 204; TYU: 84, 198 ${ }^{\mathrm{IG}}$; VGG: 157, 198, 212, 220, 223 , 328, 330; VLA: 173; VOR: 171, 248; ZAB: 198.

Trichia flavicoma (Lister) Ing - AL: 198, 215; ALT: 301, 307, 308; BA: 50, 198; CR: 244; KHM: 47, 58; KO: 50; KYA: 122, 124; SVE: 51, 198, 236; TYU: $198^{\mathrm{IG}}$.

Trichia lutescens (Lister) Lister - AL: 3, 215; BA: 50, 54, 198; CHU: 196, 198, 210, 212, 290; CR: 244; KDA: 84; KO: 50, 198; KR: 130, 198, 212, 254; KYA: 196, 198, 210, 211, 212, 290; MAG: 288; MOS: 6, 7, 8, 9, 66, 70, 84, 164, 198, 278; MUR: 196, 198; NVS: 301, 308; PER: 50; PRI: 69, 74; SVE: 50, 198, 201, 212, 236; TOM: 132 , 198; TVE: 76, 136; YAN: 50, 196, 198, 210, 212, 290.

Trichia mirabilis Nann.-Bremek. - CR: 148.

Trichia munda (Lister) Meyl. - BU: 198; CHU: 196, 198, 210, 212, 290; CR: 242, 243; IRK: 198; KO: 50; KR: 198; KYA: 196, 198, 210, 211, 212, 290; MAG : 288; MOS: 66, 164; PER: 50; PRI: 218; VGG: 27, 127, 337; YAN: 50, 196, 198, 210, 212, 290.

Trichia papillata Adamonyte - MOS: 81.

Trichia persimilis P.Karst. - AL: 215; ALT: 301, 308; CR: 244; DA: 162; IRK: 84; KLU: 84; KYA: 10; LEN: 89, 101, 198; MOS: 7, 63, 66, 70, 84, 101, 164, 198; MUR: 65, 67; NVS: 301, 308; PRI: 74, 84, 218; PSK: 73; SMO: 101, 198; TA: 101, 198; TOM: 132, 198; TVE: 19, 76, $84,141$.

Trichia scabra Rostaf. - AL: 3, 134, 215; ALT: 215, 301, 302, 306, 308, 316, 317; BA: 50, 54, 198; CHE: 50, 198; CR: 30, 84, 244; IRK: 84; KDA: 198; KHM: 47, 52,
58; KIR: 14, 267, 268, 269; KLU: 8, 101, 198; KO: 50, 198; KR: 130, 198; KRS: 5, 198; KYA: 10, 120, 122, 123 , 124, 198; LEN: 101, 180, 182, 183, 195, 198, 212, 237; LIP: 198, 252; MOS: 1, 6, 7, 8, 9, 11, 64, 66, 70, 84, 93, 94, 101, 164, 175, 198, 278; MUR: 65, 67; NVS: 301 , 308; PER: 50; PRI: 21, 74, 218; PSK: 73, 198; RYA: 101, 198; SAM: 154; SMO: 101, 198; STA: 198; SVE: 49, 50, 198, 201, 212, 236; TA: 198; TOM: 132, 198; TVE: 6, 19 , $76,84,136,141,179,182,198,204$; TYU: $198^{\mathrm{IG}}$; VGG: 157, 198, 212, 220, 223, 328, 338; VOR: 167, 171, 248.

Trichia sordida Johannesen - KC: 34, 217, 255; KHM: 47, 58; TYU: $198^{1 \mathrm{IG}}$.

Trichia subfusca Rex - AL: 215; BU: 198; KHM: 47, 52, 58; KO: 50, 198; KR: 198, 212, 254 ${ }^{\mathrm{CF}}$; KYA: 122, 124; MOS: 6, 7, 66, 70, 198; MUR: 65; NVS: 301, 308; PER: 50; PRI: 69, 74, 218; SVE: 198, 201, 212, 236; TVE: 19, 141; TYU: $198^{\mathrm{IG}}$.

Trichia varia (Pers. ex J.F.Gmel.) Pers. - AL: 3, 56, 134, 191, 214, 215; ALT: 56, 215, 301, 302, 306, 308, 316, 317; ARK: 143; AST: 198, 220, 223, 327, 328, 333; BA: 50, 54, 198; BRY: 16, $101^{\text {OAU; }}$ CHE: 50, 198; CHU: 196, 198, 210, 212, 290; CR: 30, 84, 144, 241, 242, 243 , 244; DA: 162; IRK: 84; KC: 56, 57, 97, 98, 101, 198; KDA: 78, 84, 198; KHA: 84, 198; KHM: 47, 52, 58; KIR: 263, 264, 265, 267, 272, 274, 275, 276, 277, 291; KK: $250^{\mathrm{IG}}, 294^{\mathrm{IG}}$; KLU: 101, 198; KO: 50, 198; KR: 143, 198, 212, 254; KRS: 5, 185; KYA: 10, 101, 112, 122, 124, 129 , 196, 198, 210, 211, 212, 250 $0^{\mathrm{IG}}, 290,294^{\mathrm{IG}}$; LEN: 89, 101, 180, 182, 183, 195, 198, 199, 212, 237, 323; LIP: 198 , 252; MAG: 290; MOS: 4, 6, 7, 8, 9, 11, 18, 64, 66, 70, 84, 94, 101, 159, 160, 164, 175, 198, 278; MUR: 65, 67, 84; NGR: 101, 198; NIZ: 101, 176, 198; NVS: 56, 301, 308; ORE: 50; ORL: 168, 198; PER: 50; PRI: 21, 74, 203, 218; PSK: 73, 101, 140, 198; ROS: 147, 335; SAM: 153 , 154; SMO: 99, 100, 101, 198; STA: 198; SVE: 49, 50, 51, 198, 201, 212, 234, 236; TA: 101, 198, 262; TOM: 114 , 115, 132, 198; TVE: 19, 76, 84, 136, 141, 180, 182, 198 , 204; TYU: 84, 198 ${ }^{\mathrm{IG}}$; VGG: 157, 198, 212, 220, 223, 283 , 328 , 338; VLA: 173; VOR: $167,171,239,248,249$; YAN: 196, 198, 210, 290.

Trichia verrucosa Berk. - AL: 134; KO: 50, 198; KYA: 120, 122, 123, 124; MOS $^{\mathrm{DR}}$ : 6, $7^{\mathrm{CF}}$, 198; SVE: 39, 50, 198; TVE: 19, 136, 166, 180, 181, 198, 204. 
Trichioides iridescens Novozh., Hooff \& Jagers KC: 208; SAK: 208.

Tubifera applanata (Leontyev \& Fefelov) Leontyev \& Fefelov - CHE: 146; CR: 146; KHA: 146; KO: 146; KR: 84; MOS: 84; PSK: 73; SVE: 146; TVE: 19, 76.

Tubifera dudkae (Leontyev \& G.Moreno) Leontyev, G.Moreno \& Schnittler - NVS: 149.

Tubifera ferruginosa (Batsch) J.F.Gmel. - AL: 3, 134, 149, 191, 215; ALT: 215, 299, 301, 302, 306, 308, 316; ARK: 143; BA: 50, 198; BRY: 16, $101^{\mathrm{OAU}}, 126$; CHE: 50, 198; CR: 149, 244; DA: 162; KDA: 90; KHA: 198; KHM: 47, 52, 58; KIR: 263, 265, 267, 268; KLU: 101, 198; KO: 50, 198; KR: 96, 198, 212, 254; KRS: 5, 185, 198; KYA: 10 , 122, 124, 129, 198; LEN: 89, 96, 101, 180, 182, 183, 195, $198,199,212,237,286^{\mathrm{DS}}, 287^{\mathrm{DS}}, 323$; LIP: 198, 252; MAG: 198, 290; MOS: 1, 2, 4, 6, 7, 8, 9, 11, 18, 63, 64, 66, 70, 84, 92, 101, 158, 164, 175, 198, 278; MUR: 65, 84, 196, 198, 205, 210, 212, 290; NVS: 149, 301, 308; ORL: 168; PER: 50; PRI: 21, 22, 74, 203; PSK: 73, 140; ROS: 198, 220, 223, 335; SAM: 156; SMO: 101, 198; SVE: 40, 41, 50, 51, 198, 201, 212, 236; TA: 101, 198, 262; TOM: 132, 198; TVE: 6, $19,76,84,101^{\mathrm{OAU}}, 135,136,141,142,179,180,182,198$, 204, 296; TYU: 198 ${ }^{\mathrm{IG}}$; ULY: 101; VGG: 198, 212, 220, 223, 328, 338; VLA: 173; VOR: 167, 171, 248.

Tubifera microsperma (Berk. \& M.A.Curtis) G.W.Martin - AL: 3, 198, 215 ${ }^{\mathrm{CF}}$, 300; LEN: 180, 181, 182, 198; MOS: 4, 7, 66 ${ }^{\mathrm{CF}}$; PSK: 140; SVE: 198, $201^{\mathrm{CF}}$, $212^{\mathrm{CF}}$; TVE: 19, 136, 180, 181, 182, 198, 204.

Willkommlangea reticulata (Alb. \& Schwein.) Kuntze - ALT: $310^{\mathrm{IG}}$, 317; KHM: 58; KYA: 120, 122, 123, 124, 198; MOS: 4, 6, 7, 66, 70, 198; NVS: $301^{\mathrm{CF}}$, 308, 310 ${ }^{\mathrm{IG}}$; SVE: $51,198$.

\section{List of publications reporting regional diversity studies}

This list contains all literature sources that contain information about the diversity of myxomycetes in each region of Russia (abbreviations of the names of regions are given in Table 2 ).
$\mathrm{AD}$ - 198, 339; AL - 3, 37, 56, 84, 133, 134, 149, 191, 198, 209, 214, 215, 233, 300, 318; ALT - 27, 56, 84, 134, 194, 209, 215, 225, 226, 299, 301, 302, 303, 306, $307,308,310,315,316,317,318$; AMU - 101; ARK 143, 339; AST - 84, 101, 198, 209, 213, 220, 221, 222, 223, 224, 225, 226, 282, 327, 328, 329, 330, 333, 339; BA - 46, 50, 54, 101, 198, 253; BEL - 12; BRY - 16, 101, 111, 126, 198; BU - 194, 198, 305, 318; CE - 57, 97, 98, 101, 198; CHE - 50, 53, 101, 146, 198; CHU - 189, 196, 198, 206, 210, 212, 290; CR - 29, 30, 84, 101, 144, $145,146,148,149,150,174,186,187,190,192,198$, 241, 242, 243, 244, 257; CU - 101; DA - 57, 97, 98, 162; IRK - 33, 84, 198, 232; IVA - 198; KAM - 198, 219, 251, 297; KB - 101; KC - 34, 56, 57, 97, 98, 101, 198, 208, 209, 216, 217, 225, 226, 255, 258, 259, 339; KDA - 68, 77, 78, 84, 90, 101, 163, 190, 192, 194, 198, 319, 339; KEM - 109; KGD - 91; KGN - 101, 339; KHA - 84, 146, 198; KHM - 26, 47, 52, 58, 196, 313, 314; KIR - 14, 107, 108, 263, 264, 265, 266, 267, 268, 269, 271, 272, 273, 274, 275, 276, 277, 291; KK - 250, 292, 293, 294, 295; KL - 198, 209, 220, 222, 223, 226, 328; KLU - 8, 84, 101, 198, 246; KO - 50, 146, 198, 270, 339; KOS - 101, 198; KR - 34, 35, 84, 96, 103, 104, 130, 143, 149, 198, 209, 212, 226, 240, 254, 259, 339; KRS - 5, 12, 101, 185, 198, 209, 226, 339; KYA - 10, 101, 103, 112, 113, 116, 118, 119, 120, 121, 122, 123, 124, 129, 194, 196, 198, 206, 209, 210, 211, 212, 226, 250, 290, 292, 293, 294, 295; LEN - 34, 35, 59, 62, 87, 89, 96, 101, 161, 180, 181, 182, $183,188,194,195,197,198,199,209,212,226,237$, 245, 246, 247, 258, 259, 261, 282, 286, 287, 322, 323; LIP - 101, 198, 252; MAG - 198, 288, 290; ME - 101; MO - 101; MOS - 1, 2, 4, 6, 7, 8, 9, 11, 15, 18, 28, 62, 63, $64,66,70,81,84,92,93,94,95,101,138,158,159,160$, 164, 175, 179, 198, 238, 278, 279, 289; MUR - 34, 36, 65, 67, 84, 96, 104, 106, 196, 198, 205, 210, 212, 258, 259, 290, 319, 339; NGR - 101, 198; NIZ - 101, 102, 176, 198, 227, 228, 229, 230; NVS - 26, 27, 56, 149, 198, 209, 226, 233, 301, 303, 307, 308, 309, 310, 311, 312, 318, 319, 320; ORE - 50, 53, 101, 198, 202; ORL - 168, 198; PER - 50, 198; PNZ - 101, 198; PRI - 17, 21, 22, 69, 74, 84, 163, 177, 194, 198, 203, 218; PSK - 73, 84, 101, 140, 198, 209, 226, 339; ROS - 101, 147, 149, 198, 220, 223, 335; RYA - 84, 101, 125, 198, 321; SA - 231, 288, 298, 326, 339; SAK - 198, 208; SAM - 101, 153, 154, 155, 156, 198; SAR - 101, 198; SE - 198, 339; SMO - 99, 100, 101, 198; STA - 57, 97, 98, 101, 198, 209, 226; SVE - 39, $40,41,42,43,44,45,46,48,49,50,51,53,55,101,146$, 149, 198, 201, 209, 212, 226, 234, 235, 236; TA - 101, 
198, 262; TAM - 101, 198; TOM - 114, 115, 117, 132, 133, 198, 233; TVE - 6, 19, 20, 75, 76, 79, 80, 84, 101, $135,136,137,138,139,141,142,165,166,179,180$, 181, 182, 198, 204, 282, 296; TY - 304; TYU - 84, 101, 198, 339; ULY - 101, 198; VGG - 27, 60, 88, 101, 110, $127,128,157,198,207,209,212,220,223,224,225$, 226, 257, 280, 281, 282, 283, 284, 285, 324, 325, 328, 329, 330, 331, 332, 334, 336, 337, 338; VLA - 6, 84, 85, 172, 173, 198; VLG - 101, 198; VOR - 25, 32, 101, 167, 169, 170, 171, 192, 198, 239, 248, 249, 339; YAN - 50, 196, 198, 210, 212, 290; YAR - 6, 59, 84, 101, 198; ZAB $-26,101,105,194,198$.

\section{Myxomycete diversity in Russia}

According to the bibliographic sources, a total of 455 myxomycete species (recognized according to a morphological species concept) from 56 genera have been recorded in Russia to date, which comprises approximately $40 \%$ of the known global diversity. So far, no specimens of the following genera have been found in Russia: Alwisia, Arcyriatella, Calonema, Cornuvia, Echinosteliopsis, Elaeomyxa, Leptoderma, Minakatella, Paradiachea, Physarina, Semimorula, and Trabrooksia. However, it should be noted that environmental nucleotide sequences $92-93 \%$ similar to Echinosteliopsis oligospora D.J. Reinh. \& L.S.Olive and clustering together with this species in 18S rDNA phylogeny were found in samples of forest ground litter from the Leningrad region (Shchepin et al. 2019a).

Out of the 455 known species, 319 species (70\%) have been reported for the first time over the last 40 years and 120 species $(26 \%)$ - over the last 15 years (Figure 1).

The most widespread and common species in Russia are Lycogala epidendrum (L.) Fr. (54 regions), Fuligo septica (L.) F.H.Wigg. (53 regions), and Stemonitis axifera (Bull.) T.Macbr. (51 regions). Almost half of species (226 out of 455) are found in 5 or fewer regions.

Myxomycetes have been recorded in 76 out of 82 regions of Russia. Despite the increased interest in myxomycetes, there are still no data on biodiversity of this group in Tula and Omsk regions,
Republic of Ingushetia, Udmurt Republic, Nenets autonomous area, and Jewish autonomous region. In addition, for 21 regions there are fewer than 10 species known (Table 2, Figure 2). Further research concerning aforementioned regions would be highly relevant.

\section{Conclusions}

The presented data indicate that the research of myxomycete diversity in Russia is still fragmentary, regarding the coverage of different regions. Only a few regions, like Moscow, Leningrad, Sverdlovsk, Tver and Volgograd regions, Altai Republic, and Altai and Primorye territories have been surveyed more exhaustively. It would require much more effort to reveal the real spatial distribution of myxomycetes. It would be even more laborious to take the hidden diversity in the group into account (amoebal populations that do not or rarely fruit in a given habitat, which thus can only be studied via metabarcoding). This annotated list is a part of a larger information system, available online at russia.myxomycetes.org. We plan to maintain this website and expand its functionality in the future. 
Figure 1. Timeline of the myxomycete diversity research in Russia.

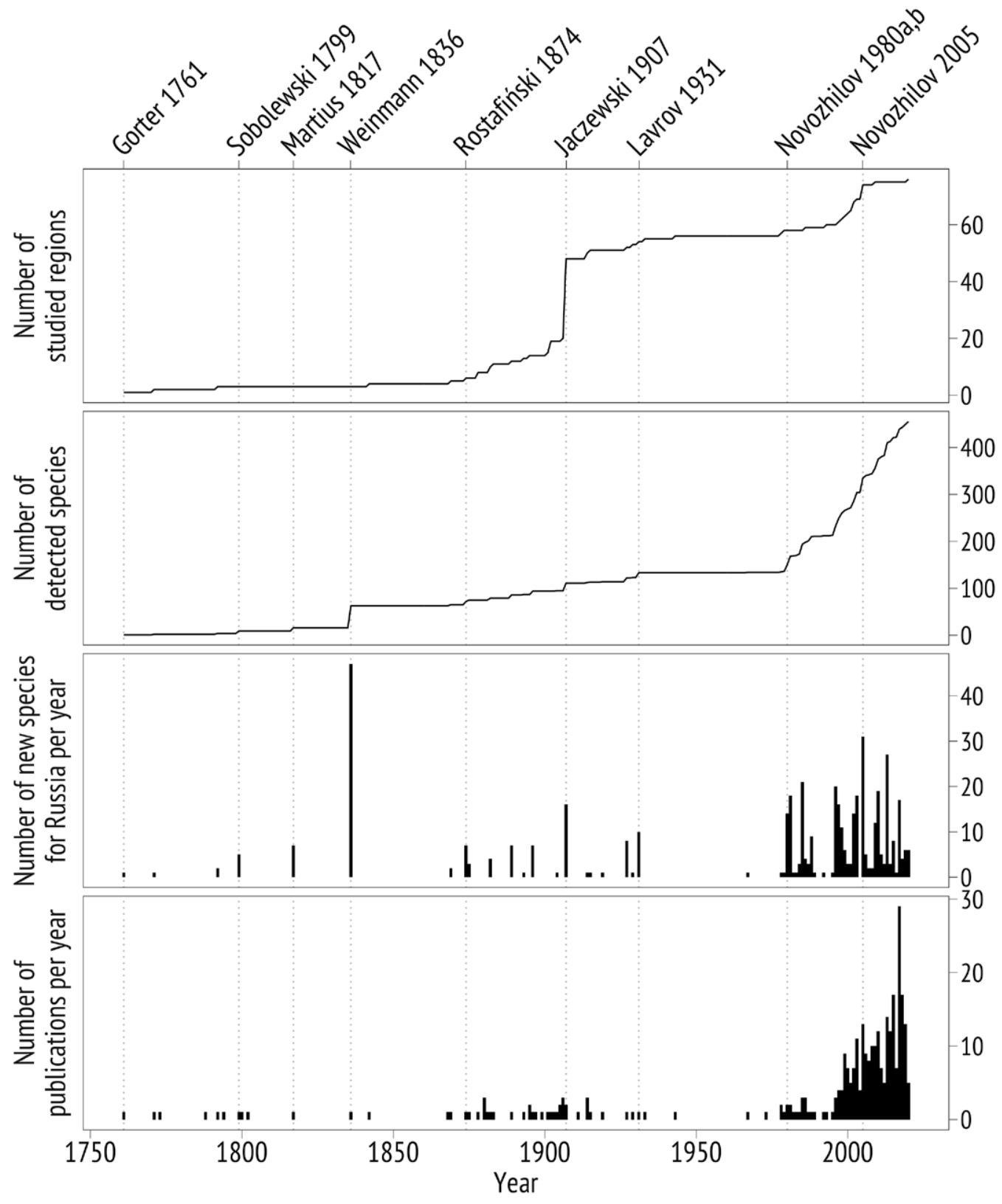


Figure 2. Number of myxomycete species found in the regions of Russia. Note: abbreviations of region names are given in accordance with Table 2.

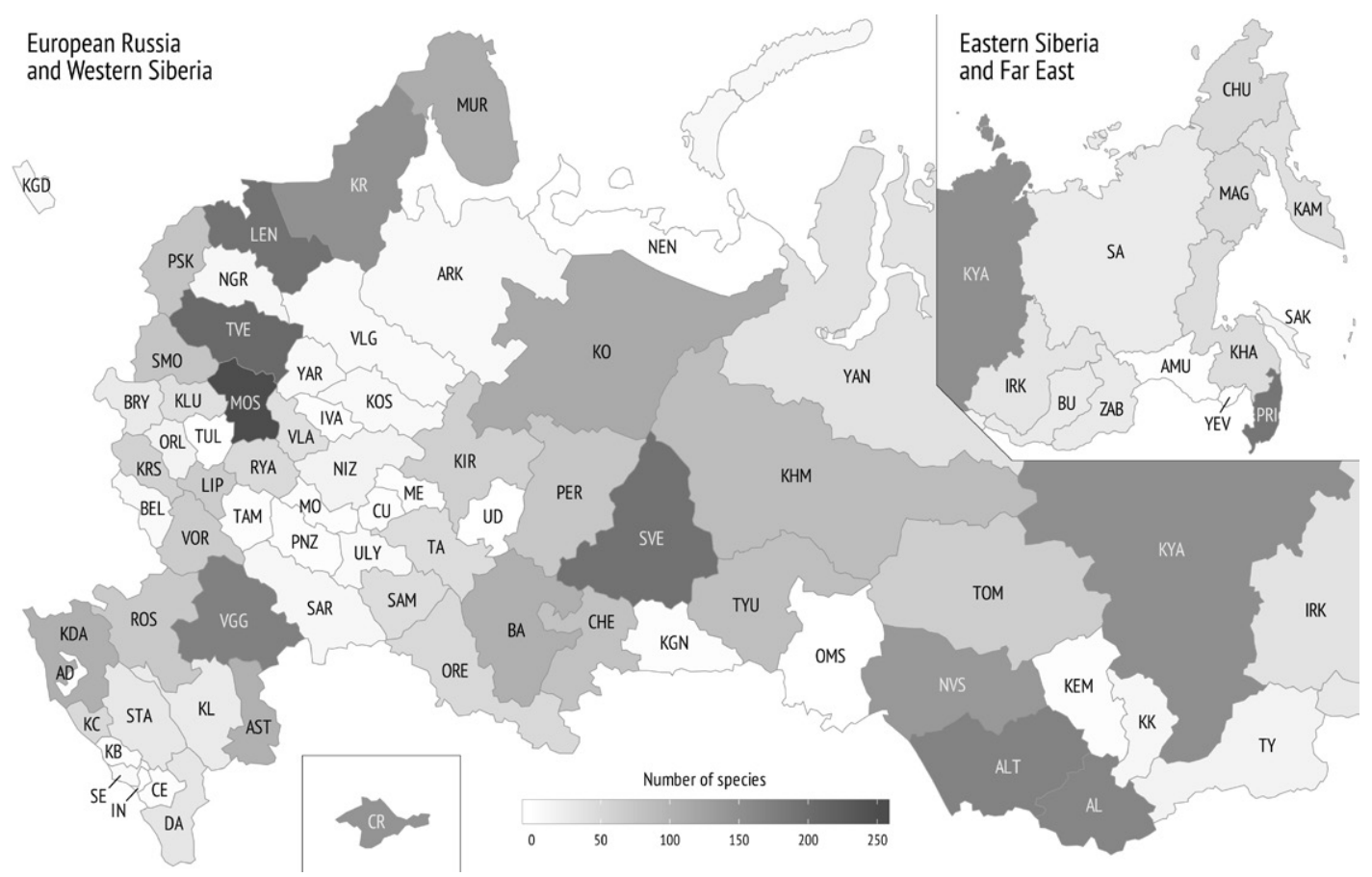

\section{Acknowledgements}

We are grateful to all researchers of myxomycete diversity in Russia that have contributed to the myxomycetology over the last 20 years, despite the fact that some of them have changed their occupation: M.F. Akimova, N.I. Borzov, V.N. Botyakov, N.Yu. Buchtoyarova, D.A. Erastova, K.A. Fefelov, E.R. Galiyakhmetova, M.M. Gomzhina, E.S. Gubanov, E.S. Korchikov, A.P. Kosheleva, A.N. Lebedev, A.D. Luptakova, G.M. Melkumov, Mishulin, A.A., Yu.A. Morozova, A.M. Nikitina, M.V. Okun, I.S. Prikhodko, B.S. Plotnikov, A.A. Shirokikh, Yu.A. Smolnyakova, A.A. Soldatenkova, E.N. Vinogradskaya, A.A. Vorobyov. We would also like to thank all school clubs that donated to us materials collected during summer fieldwork, in particular, the Young Naturalists Club under the guidance of E.A. Dunayev and the A.S. Khizhnyakova's Young Nature Researchers Club from Moscow. Research of V.I.G. was supported by the Moscow State University Grant for the Leading Scientific Schools "Depository of the Living Systems" within the scope of the MSU Development Program and the state task of MSU, part 2 (topic number AAAA-A6-116021660084-1).

O.N.S. and Yu.K.N. would like to acknowledge the support from the Russian Foundation for Basic Research (project 18-04$01232 \mathrm{~A}$ ) and the state task of BIN RAS 'Biodiversity, ecology, structural and functional features of fungi and fungus-like protists' (AAAA-A19-119020890079-6). 


\section{References}

1 Abramov, E.A., Adashev, V.E., Aksenova, J.V., Bessonova, I.D., Broshevitzkaya, N.D., Gebruk, A.A., Zornikova, K.V., Izyumova, E.I., Koneeva, T.O., Sivova, I.N., Khamidullina, A.I. \& Chursanova, E.N. 2014: Myxomycetes of Zvenigorodskaya Biological Station: species diversity in 2013 (in Russian with English abstract). In: Flora and fauna of the western part of Moscow region. Selected student research works conducted at summer field practice at S.N. Skadovsky Zvenigorod Biological Station, Vol. 7, 44-58. ZBS MSU: Zvenigorod.

2 Adashev, V.E., Aksenova, J.V. \& Gebruk, A.A. 2014: Time-lapse photography of Myxomycetes life cycle stages methods development (in Russian with English abstract). In: Flora and fauna of the western part of Moscow region. Selected student research works conducted at summer field practice at S.N. Skadovsky Zvenigorod Biological Station, Vol. 7, 59-64. ZBS MSU: Zvenigorod.

3 Barsukova, T.N. 2000: Myxomycetes from Teletskoe lake vicinity, Altai State Reserve (in Russian with English abstract). Mikologiya i Fitopatologiya 34: 6-9.

4 Barsukova, T.N. 2001a: Ecological-biological peculiarities of myxomycetes on Biological Station in Zvenigorod (in Russian with English abstract). Trudy Zvenigorodskoy Biologicheskoy Stantsii 3: 90-91.

5 Barsukova, T.N. 2001b: Myxomycetes of the Aliokhin Central Chernozem Biosphere Reserve (in Russian with English abstract). Mikologiya i Fitopatologiya 35: 12-14.

6 Barsukova, T.N. \& Dunayev (Dunaev), E.A. 1997: An annotated list of slime moulds (Myxomycota) from Moscow region (in Russian with English abstract). Mikologiya i Fitopatologiya 31: 1-8.

7 Barsukova, T.N., Gmoshinskii, V.I., Prokhorov, V.P. \& Dunaev, E.A. 2012: The slime moulds of S.N. Skadovsky Zvenigorod Biological Station of Moscow State University (in Russian with English abstract). Mikologiya i Fitopatologiya 46: 122-132.

8 Barsukova, T.N., Prokhorov, V.P., Gmoshinskii, V.I. \& Chizhov, A.O. 2010: Myxomycetes in forest parks of Moscow, Moscow region, and some areas of the Kaluga region. Moscow University Biological Sciences Bulletin 65: 116-118.
9 Barsukova, T.N., Vinogradskaya, E.N. \& Akimova, M.F. 2006: Myxomycetes in forestparks in Moscow (in Russian with English abstract). Mikologiya i Fitopatologiya 40: 186-189.

10 Beglyanova, M.I. \& Kattsyna, L. 1973: Myxomycetes of the southern Krasnoyarsk territory (in Russian). In: Gorbunova, T.V. (ed). Questions of botany and plant physiology, Vol. 4, 36-47. Krasnoyarskiy rabochiy, Krasnoyarsk.

11 Belyaeva, M.A., Zakirov, A.N., Rusanova, V.R., Suchalko, O.N., Filippova, A.A., Khitiaeva, V.V., Shmitko, A.O. \& luzefovich, A.P. 2017: Results of myxomycetes species diversity in Skadovsky Zvenigorod Biological Station of MSU in 2015 (in Russian with English abstract). In: Gavrilov, V.M. (ed). Flora and fauna of the western part of Moscow region. Selected student research works conducted at summer field practice at S.N. Skadovsky Zvenigorod Biological Station, Vol. 8, 7-22. Moscow.

12 Benike, L.A. 1915: First report on flora of slime fungi from Kharkov and Kursk Governorates (in Russian). Protocoly Obshchestva ispytateley prirody pri Imperatorskom Kharkovskom universitete $3: 1-3$.

13 Berestetskaya, L.I., Dmitriev, A.P. \& Levitin, M.M. 2012: Mycological Herbarium of All-Russian Institute of Plant Protection (in Russian). Plant Protection News 3: 58-61.

14 Berezina, Yu.S. \& Shirokikh, A.A. 2017: Slime moulds of subclass Myxogastria in forest communities and their culture in vitro (in Russian). In: Ekologiya rodnogo kraya: problemy i puti ikh resheniya. Materialy XII Vserossiyskoy nauchno-prakticheskoy konferentsii c mezhdunarodnym uchastiem. Kniga 2. (g. Kirov, 13-14 aprelya 2017 g.), 276-279. Vyatka State University: Kirov.

15 Bodyagin, V.V. \& Barsukova, T.N. 2009: Myxomycetes isolated from aquatic habitats of Moscow city and Moscow region (in Russian with English abstract). Mikologiya i Fitopatologiya 43: 281-283.

16 Borščow, E. 1869: Ein Beitrag zur Pilzflora der Provinz Černigow (in German). Bulletin de l'Académie Impériale des sciences de St-Pétersbourg 13: 219-245.

17 Bortnikov, F.M., Shchepin, O.N., Gmoshinskiy, V.I., Prikhodko, I.S. \& Novozhilov, Yu.K. 2018: Diderma velutinum, a new species of Diderma (Myxomycetes) with large columella and triple peridium from Russia. Botanica Pacifica 7: 47-51.

18 Bucholtz, F.V. 1897: List of fungi found in summer of 1896 (in Russian). Typolithography of I.N. Kushnerev and Co partnership: Moscow. 
19 Buchtoyarova, N.Yu. \& Gmoshinskiy, V.I. 2017: Results of the study of myxomycete (class Myxomycetes) species diversity in the southern forestry of the Central Forest Nature Reserve from 2014 to 2016 (in Russian). In: Vklad zapovednoy sistemy $v$ sokhranenie bioraznoobraziya i ustoychivoe razvitie. Materialy Vserossiyskoy nauchnoy konferentsii (s mezhdunarodnym uchastiem), posvyashchennoy 85-letiyu organizatsii TsLGPBZ i 100 -letiyu zapovednoy sistemy Rossii, 69-75. Tver State University: Tver.

20 Buchtoyarova, N.Yu., Gmoshinskiy, V.I. \& Matveev, A.V. 2018: The results of the long-term monitoring of the species diversity of myxomycetes in the Central Forest National Biosphere Reserve (in Russian and English). In: Proceedings of IV (XII) International Botanical Conference of Young Scientists in Saint-Petersburg, April 22nd-28th, 2018, 202-203. Komarov Botanical Institute of the Russian Academy of Sciences: St. Petersburg.

21 Bunkina, I.A. 1978: Fungi (in Russian). In: Kharkevich, S.S. (ed). Flora and vegetation of the Ussuriyskiy Nature Reserve, 36-39. Nauka, Moscow.

22 Bunkina, I.A. \& Koval, E.Z. 1967: Ad floram myxomycetum e regione Primorskensi [On flora of myxomycetes of Primorsky territory] (in Russian). Novosti sistematiki nizshikh rastenii 4: 152-153.

23 Buxbaum, J.C. 1740: Plantarum minus cognitarum centuria $\mathrm{V}$, complectens plantas circa Byzantium et in Oriente observatas (in Latin). Typographia Academiae: Petropoli.

24 Carine, M.A., Cesar, E.A., Ellis, L., Hunnex, J., Paul, A.M., Prakash, R., Rumsey, F.J., Wajer, J., Wilbraham, J. \& Yesilyurt, J.C. 2018: Examining the spectra of herbarium uses and users. Botany Letters 145: 328-336.

25 Castillo, A., Illana, C. \& Moreno, G. 1998: Protophysarum phloiogenum and a new family in the Physarales. Mycological Research 102: 838-842.

26 Czernyadjeva, I.V., Afonina, O.M., Ageev, D.V., Baisheva, E.Z., Bulyonkova, T.M., Cherenkova, N.N., Doroshina, G.Ya., Drovnina, S.I., Dugarova, O.D., Dulepova, N.A., Dyachenko, A.P., Filippova, N.V., Ginzburg, E.G., Gogorev, R.M., Himelbrant, D.E., Ignatov, M.S., Kataeva, O.A., Kotkova, V.M., Kuragina, N.S., Kurbatova, L.E., Kushnevskaya, E.V., Kuzmina, E.Yu., Melekhin, A.V., Notov, A.A., Novozhilov, Yu.K., Popov, S.Yu., Popova, N.N., Potemkin, A.D., Stepanchikova, I.S., Stepanova, V.A., Tubanova, D.Ya., Vlasenko, A.V., Vlasenko, V.A., Voronova, O.G. \& Zhalov, Kh.Kh. 2019: New cryptogamic records. 4 (in Russian with English abstract). Novosti sistematiki nizshikh rastenii 53: 431-479.
27 Czernyadjeva, I.V., Kotkova, V.M., Zemlyanskaya, I.V., Novozhilov, Yu.K., Vlasenko, A.V., Vlasenko, V.A., Blagoveshchenskaya, E.Yu., Georgieva, M.L., Notov, A.A., Himelbrant, D.E., Muchnik, E.E., Urbanavichene, I.N., Aristarkhova, E.A., Bocharnikov, M.V. \& Ismailov, A.B. 2018: New cryptogamic records. 2 (in Russian with English abstract). Novosti sistematiki nizshikh rastenii 52: 209-223.

28 Dokturovskiy, V.S. 1905: Minutes of meetings of the Student Circle for Study of Russian Nature (in Russian). In: Proceedings of the Student Circle for the Study of Russian Nature, affiliated to the Moscow Imperial University. Book 2, 3-12. Typolithography of Russian Partnership of Printing and Publishing, Moscow.

29 Dudka, I.O. 2000: New for Ukraine nivale myxomycete species from Crimea (in Ukrainian with Russian and English abstract). Ukrainian Botanical Journal 57: 57-61.

30 Dudka, I.A., Kuzub, V.V. \& Romanenko, E.A. 1999: Myxomycetes of the Yalta Mountain-forest reserve (Ukraine, Crimea) (in Russian with English abstract). Mikologiya i Fitopatologiya 33: 307-313.

31 Dunayev, E.A. \& Barsukova, T.N. 2002: Slime-mould collection (Myxomycota) of the Young Naturalists Club of the Zoological Museum of Moscow State University (in Russian). In: Sovremennaya mikologiya v Rossii. Tom 1. Materialy 1-go Syezda mikologov Rossii, 132. National Academy of Mycology: Moscow.

32 Eliasson, U. \& Gilert, E. 1982: A SEM-study of Listerella paradoxa (Myxomycetes). Nordic Journal of Botany 2: 249-255.

33 Eliasson, U. \& Lundqvist, N. 1979: Fimicolous myxomycetes. Botaniska notiser 132: 551-568.

34 Erastova, D.A. 2015: Nivicolous myxomycetes of NorthWestern Russia and North-Western Caucasus (in Russian). PhD thesis. V.L. Komarov BIN RAS: St. Petersburg.

35 Erastova, D.A. \& Novozhilov, Yu.K. 2015: Nivicolous myxomycetes of the lowland landscapes of the northwest of Russia. Mikologiya i Fitopatologiya 49: 9-18.

36 Erastova, D.A., Novozhilov, Yu.K. \& Schnittler, M. 2017: Nivicolous myxomycetes of the Khibiny Mountains, Kola Peninsula, Russia. Nova Hedwigia 104: 85-110.

37 Erastova, D.A., Okun, M.V., Novozhilov, Yu.K. \& Schnittler, M. 2013: Phylogenetic position of the enigmatic myxomycete genus Kelleromyxa revealed by SSU rDNA sequences. Mycological Progress 12: 599-608. 
38 Falk, J.P. 1786: Beytrage zur topographischen Kenntniss des Russischen Reichs, Bd. II (in German). Kaiserliche Akademie der Wissenschaften: St. Petersburg.

39 Fefelov, K.A. 1997a: Impact of aerotechnogenic pollution by heavy metals and $\mathrm{SO}_{2}$ on myxomycetes (in Russian). In: Bezopasnost biosfery. Sbornik tezisov dokladov Vserossiyskogo molodezhnogo nauchnogo simpoziuma, 221. Ukhta State Technical University: Yekaterinburg.

40 Fefelov, K.A. 1997b: Myxomycetes as a component of forest ecosystems (in Russian). In: Problemy izucheniya bioraznoobraziya na populyatsionnom i ekosistemnom urovne. Materialy konferentsii molodykh uchenykh-ekologov Uralskogo regiona 1-4 aprelya 1997 g., 237-239. Yekaterinburg.

41 Fefelov, K.A. 1997c: Species composition of myxomycetes (in Russian). In: Vertical. Bulletin of young science of the Urals, Vol. 2, 64-66. AMUiS, Orenburg.

42 Fefelov, K.A. 1998a: Myxomycetes of the city of Yekaterinburg (in Russian). In: Bezopasnost biosfery. Sbornik tezisov dokladov II Vserossiyskogo molodezhnogo nauchnogo simpoziuma, 102. Ukhta State Technical University: Yekaterinburg.

43 Fefelov, K.A. 1998b: Slime moulds of burnt areas (in Russian). In: Bezopasnost biosfery. Sbornik tezisov dokladov II Vserossiyskogo molodezhnogo nauchnogo simpoziuma, 107. Ukhta State Technical University: Yekaterinburg.

44 Fefelov, K.A. 1999a: Corticolous myxomycetes of Sverdlovsk region (in Russian). In: Chereshiev, V.A. \& Kuyukina, M.S. (eds). Sovremennye problemy ekologii, mikrobiologii i immunologii. Materialy regionalnoy konferentsii molodykh uchenykh 18-20 yanvarya 1999 goda, Perm, 63-65. Perm.

45 Fefelov, K.A. 1999b: Reaction of myxomycete communities in gradient of aerotechnogenic pollution (in Russian). In: Razvitie idey akademika S.S. Shvartsa v sovremennoy ekologii. Sbornik trudov konferentsii molodykh uchenykh-ekologov Uralskogo regiona 2-3 aprelya 1999 goda, 201-205. Yekaterinburg: Yekaterinburg.

46 Fefelov, K.A. 2002a: Myxomycetes of anthropogenically disturbed territories (in Russian with English abstract). In: Storozhenko, V.G. \& Selochnik, N.N. (eds). Problems of forest phytopathology and mycology. Proceedings of the 5th International Conference, 7-10 (13) October 2002, 254-256. Moscow.

47 Fefelov, K.A. 2002b: Myxomycetes of the "Sibirskie Uvaly" Natural Park (West-Siberian Plain) (in Russian with English abstract). In: Shor, E.L. (ed). Ekologicheskiye issledovaniya vostochnoy chasti Sibirskikh Uvalov. Sbornik nauchnykh trudov zapovedno-prirodnogo parka "Sibirskiye Uvaly", Vol. 1, 93-103. Priobye, Nizhnevartovsk.

48 Fefelov, K.A. 2002c: Some interesting species of the myxomycetes from the city (in Russian with English abstract). In: Ryabinina, Z.N. (ed). Biodiversity and bioresources of Urals and adjacent territories. Materials of II International Conference, Orenburg, December 17-18, 2002, 34-35. Orenburg State Pedagogical University: Orenburg.

49 Fefelov, K.A. 2003: Myxomycetes (in Russian). In: Gorchakovskiy, P.L. (ed). Plants and fungi of the Pripyshminskie Bory National Park, 96-98. Ural University Publishing House, Yekaterinburg.

50 Fefelov, K.A. 2005: Myxomycetes (class Myxomycetes) of Ural: taxonomical composition, ecology, geography (in Russian). PhD thesis. Institute of Plant and Animal Ecology of the Ural Branch of the Russian Academy of Sciences (IPAE UB RAS): St. Petersburg

51 Fefelov, K.A. 2006: Annotated list of myxomycetes of Visim Nature Reserve (in Russian). In: Marin, Yu.F. (ed). Ekologicheskie issledovaniya $v$ Visimskom biosfernom zapovednike. Materialy nauchnoy konferentsii, posvyashchennoy 35-letiyu Visimskogo zapovednika. Yekaterinburg, 2-3 oktyabrya 2006 g., 326-329. Sredne-uralskoe knizhnoe izdatelstvo. Novoe vremya: Yekaterinburg.

52 Fefelov, K.A. 2007: Myxomycetes of Malaya Sosva Nature Reserve (in Russian). In: Biological resources and nature management. Proceedings, Vol. 10, 128-133. Defis, Surgut.

53 Fefelov, K.A. 2009: Comparison of the myxomycete complexes of Ural steppes (in Russian). Vestnik of the Orenburg State University 6: 387-389.

54 Fefelov, K.A. 2010: Basic check-list of myxomycetes from Uzhno-Uralsky State Reserve (in Russian with English abstract). Proceedings of the Tigirek State Natural Reserve 3: 121-124.

55 Fefelov, K.A. \& Plotnikov, B.S. 2002: Corticolous myxomycetes of pine (Pinus sylvestris L.) in urban habitat (in Russian). In: Ivanov, A.I. (ed). Voprosy prakticheskoy ekologii. Sbornik materialov Vserossiyskoy nauchnoprakticheskoy konferentsii, 29-30 maya 2002 g., 214-216. Penza State Agricultural Academy: Penza.

56 Feng, Y. \& Schnittler, M. 2015: Sex or no sex? Group I introns and independent marker genes reveal the existence of three sexual but reproductively isolated biospecies in Trichia varia (Myxomycetes). Organisms Diversity \& Evolution 15: 631-650. 
57 Filarszky, F. 1907: Botanische Ergebnisse der Forschungsreisen von M. v. Déchy im Kaukasus (in German).

In: Déchy, M. (ed). Kaukasus. Reisen und Forschungen im kaukasischen Hochgebirge, Vol. 3, 1-126. Dietrich Reimer (Ernst Vohsen), Berlin.

58 Filippova, N.V. 2010: Preliminary list of fungi and myxomycetes of Khanty-Mansi autonomous area (in Russian). Khanty-Mansiysk.

59 Fiore-Donno, A.M., Kamono, A., Chao, E.E., Fukui, M. \& Cavalier-Smith, T. 2010: Invalidation of Hyperamoeba by transferring its species to other genera of Myxogastria. The Journal of Eukaryotic Microbiology 57: 189-196.

60 Fiore-Donno, A.M., Meyer, M., Baldauf, S.L. \& Pawlowski, J. 2008: Evolution of dark-spored Myxomycetes (slime-molds): Molecules versus morphology. Molecular Phylogenetics and Evolution 46: 878-889.

61 Georgi, J.G. 1790: Versuch einer Beschreibung der Russisch Kayserlichen Residenzstadt St. Petersburg und der Merkwurdigkeiten der Gegend, Bd. II (in German). Kaiserliche Akademie der Wissenschaften: St. Petersburg.

62 Georgi, J.G. 1800: Geographisch-physikalische und Naturhistorische Beschreibung des Russischen Reichs (in German). Friedrich Nicolovius: Königsberg.

63 Gerasimovich, E.S., Pavlova, N.S., Pletenev, A.A., Podkuichenko, N.V., Rafaeva, M.P., Teteneva, N.A., Frolov, O.Yu. \& Tsvetkova, M.S. 2014: Species diversity and distribution of myxomycetes of Zvenigorod Biological Station in 2012 year (in Russian with English abstract). In: Flora and fauna of the western part of Moscow region. Selected student research works conducted at summer field practice at S.N. Skadovsky Zvenigorod Biological Station, Vol. 7, 31-43. ZBS MSU: Zvenigorod.

64 Gluhareva, I.D., Golovaneva, M.S., Egorov, A.V., Komedchikova, E.N., Menshikova, N.K., Myagkaya, A.V., Petrushenko, T.U., Rutškin, D.A., Ustimenko, A.A. \& Hromova, M.R. 2017: Species diversity of myxomycetes in Zvenigorodskaya Biological Station of S.N. Skadowsky in 2016 (in Russian with English abstract). In: Flora and fauna of the western part of Moscow region. Selected student research works conducted at summer field practice at S.N. Skadovsky Zvenigorod Biological Station, Vol. 9, 26-43. KMK Scientific Press Ltd.: Moscow.

65 Gmoshinskiy (Gmoschinskiy), V.I. 2013a: An annotated checklist of the myxomycetes of the Poriya Guba bay (White Sea): primary report (in Russian with English abstract). In: Tolmacheva, E.L. (ed). The Chronicle of Nature of the Kandalaksha Reserve for 2013 (Annual report). Book 59, Vol. 1, 1-9. Kandalaksha.
66 Gmoshinskiy, V.I. 2013b: Myxomycetes of Moscow and Moscow region (in Russian). PhD thesis. Lomonosov MSU: Moscow.

67 Gmoshinskiy (Gmoschinskiy), V.I. 2015: The results of the study of diversity myxomycetes of the Poriya Guba bay and Luvenga area in 2014 (in Russian with English abstract). In: Tolmacheva, E.L. (ed). The Chronicle of Nature of the Kandalaksha Reserve for 2014 (Annual report).

Book 60, Vol. 1, 19-30. Kandalaksha.

68 Gmoshinskiy, V.I. 2017: Results of studying myxomycetes diversity on the territory of "Utrish" reserve in 2016 (in Russian with English abstract). In: Kalinina, S.Yu. Bykhalova, O.N. (ed). Terrestrial and adjacent marine ecosystems of the Abrau peninsula: structure, biodiversity and protection. Proceedings, Vol. 4, 134-140. I.V. Kazenin, Moscow.

69 Gmoshinskiy, V.I. \& Antonov, E.A. 2016: On the Myxomycetes biota of Primorye (in Russian with English abstract).

Bulletin of the Botanical Garden-Institute FEB RAS 15: 16-19.

70 Gmoshinskiy, V.I. \& Matveev, A.V. 2016: Seasonal dynamics of sporophore formation in myxomycetes of Moscow and Moscow region. Mikologiya i Fitopatologiya 50: 139-147.

71 Gmoshinskiy, V.I. \& Matveev, A.V. 2017: Myxomycete collection of the Department of Mycology and Algology (in Russian). In: Dyakov, Yu.T. (ed). Sovremennaya mikologiya v Rossii. Tom 6, 164-166. National Academy of Mycology, Moscow.

72 Gmoshinskiy, V.I. \& Matveev, A.V. 2018: Revision of myxomycete collection of the Zoological Museum of Moscow State University (in Russian). In: Proceedings of IV (XII) International Botanical Conference of Young Scientists in Saint-Petersburg, April 22nd-28th, 2018, 204-205. Komarov Botanical Institute of the Russian Academy of Sciences: St. Petersburg.

73 Gmoshinskiy, V.I. \& Matveev, A.V. 2019: First data on Myxomycetes of Polistovsky Nature Reserve (Pskov region). Novosti sistematiki nizshikh rastenii 53: 279-290.

74 Gmoshinskiy, V.I., Bortnikov, F.M., Matveev, A.V. \& Novozhilov, Yu.K. 2020a: New data on Myxomycetes of Lazovsky State Nature Reserve (Far East, Russia). Botanica Pacifica 9: 155-164.

75 Gmoshinskiy, V.I., Buchtoyarova, N.Yu. \& Matveev, A.V. 2017a: First record of Physarum spectabile (Myxomycetes) in Russia. Botanica Lithuanica 23: 107-110. 
76 Gmoshinskiy, V.I., Buchtoyarova, N.Yu. \& Matveev, A.V. 2017b: Study of species diversity of myxomycetes (class Myxomycetes) of the Central Forest Nature Reserve in 2015-2016 (in Russian). In: Dynamics of phenomena and processes in the natural complex of the reserve. The Chronicle of Nature. Book 56. 2016, 192-225. Central Forest State Nature Reserve: Zapovedny.

77 Gmoshinskiy, V.I., Dunaev, E.A. \& Sosorev, G.M. 2014: The first time record of Fuligo megaspora (Myxomycetes) in the territory of Russia (in Russian with English abstract). Mikologiya i Fitopatologiya 48: 209-211.

78 Gmoshinskiy, V.I., Dunayev, E.A. \& Sosorev, G.M. 2015: Results of the study [of] Mixomycota in the "Utrish" Reserve (in Russian with English abstract). In: Bykhalova, O.N. (ed). Biota protection in the State Nature Reserve "Utrish". Proceedings. Vol. 3. 2014, 195-200. Poligraf-YuG, Maykop.

79 Gmoshinskiy, V.I., Ershov, A.A., Korobkov, A.G., Lebedev, A.N., Lebedeva, M.V. \& Sinitenkova, M.A. 2013: Myxomycetes (in Russian). In: Sorokin, A.S., Zinovyev, A.V., Pushay, E.S. \& Tyusov, A.V. (eds). Red Data Book of the Tver region, 181-192. Tver.

80 Gmoshinskiy, V.I., Ershov, A.A., Korobkov, A.G., Lebedev, A.N., Lebedeva, M.V. \& Sinitenkova, M.A. 2016: Myxomycetes (in Russian). In: Orlov, S.V., Sokolov, D.L., Perova, I.S., Kokina, O.M., Turilov, A.V. \& Vedernikova, E.S. (eds). Red Data Book of the Tver region, 187-196. Tverskoy Pechatny Dvor, Tver.

81 Gmoshinskiy, V.I., Gubanov, E.S. \& Matveev, A.V. 2019a: First record of Trichia papillata Adamonyte (Myxomycetes) in Russia. Botanica 25: 16-20.

82 Gmoshinskiy, V.I., Matveev, A.V., Bortnikov, F.M. \& Adashev, V.E. 2018a: Myxomycete study at the Department of Mycology and Algology, Faculty of Biology, Moscow State University (in Russian). In: Materialy Vserossiyskoy konferentsii s mezhdunarodnym uchastiem, "Mikologiya i algologiya Rossii. XX - XXI vek: smena paradigm", posvyashchennoy 100 -letiyu kafedry mikologii i algologii biologicheskogo fakulteta MGU imeni M.V. Lomonosova, 110-letiyu so dnya rozhdeniya professora Mikhaila Vladimirovicha Gorlenko, pamyati professora Yuriya Tarichanovicha Dyakova, 52-60. Pero: Moscow.

83 Gmoshinskiy, V.I., Matveev, A.V. \& Dunaev, E.A. 2018b: Collection of myxomycetes of Young Naturalists Circle of Zoological Museum of Moscow State University. Lomonosov Moscow State University. Occurrence dataset: https:// doi.org/10.15468/zmmwmj (Date of access: 01.06.2020).
84 Gmoshinskiy, V.I., Matveev, A.V., Gubanov, E.S., Bortnikov, F.M. \& Dunayev, E.A. 2020b: Critical revision of the Myxomycetes collection of Young Naturalists Club of Zoological Museum of Moscow State University. Botanica Pacifica 9: 175-190.

85 Gmoshinskiy, V.I., Mishulin, A.A. \& Matveev, A.V. 2019b: First record of Didymium projectile (Myxomycetes) in Russia. Bulletin of Moscow Society of Naturalists. Biological series 124: 34-36.

86 Gmoshinskiy, V.I., Prokhorov, V.P. \& Barsukova, T.N. 2011: Myxomycet[e]s collection of the Department of Mycology and Algology of Moscow State University. Mikologiya i Fitopatologiya 45: 98-100.

87 Gorter, D. 1761: Flora Ingrica ex schedis Stephani Krascheninnikow confecta et propriis observationibus aucta a Davide de Gorter (in Latin). Petropoli.

88 Gorunova, A.V. \& Ebel, M.A. 2017: Members of family Stemonitidaceae in myxomycete biota of Volga-Akhtuba Flood Plain (in Russian). In: Petrov, V.I. (ed). Aktualnye problemy eksperimentalnoy $\mathrm{i}$ klinicheskoy meditsiny. $\mathrm{Ma}$ terialy 75-y otkrytoy nauchno-prakticheskoy konferentsii molodykh uchenykh i studentov VolgGMU s mezhdunarodnym uchastiem, 481-482. Volgograd State Medical University: Volgograd.

89 Grimm, M. 1896: Zur Myxomycetenkenntniss des Gouvernements St.-Petersburg [On myxomycetes of Petersburg Governorate] (in Russian). Scripta Botanica Horti Universitatis Imperialis Petropolitanae 5: 157-171.

90 Gubanov, E.S. \& Gmoshinskiy, V.I. 2018: Results of exploring species diversity of slime molds in Nature State Reserve "Utrish" in 2017 (in Russian and English). In: Proceedings of IV (XII) International Botanical Conference of Young Scientists in Saint-Petersburg, April 22nd-28th, 2018, 207. Komarov Botanical Institute of the Russian Academy of Sciences: St. Petersburg.

91 Hennings, P. 1895: Beitrag zur Pilzflora des Samlandes (in German). Jahres-Bericht des Preussischen Botanischen Vereins: 85-90.

92 Hennings, P. 1903: Beitrag zur Pilzflora des Gouvernements Moskau (in German). Hedwigia 42: 108-118.

93 Hennings, P. 1904: Zweiter Beitrag zur Pilzflora des Gouvernements Moskau (in German). Hedwigia 43: 66-73.

94 Hennings, P. 1906: Dritter Beitrag zur Pilzflora des Gouvernements Moskau (in German). Hedwigia 45: 22-33. 
95 Heyden, K.K. 1899: Zur Pilzflora des Gouvernements Moskau (in German). Hedwigia 38: 269-273.

96 Hintikka, T.J. 1919: Révision des Myxogastres de Finlande (in French). Acta Societatis pro Fauna et Flora Fennica 46: 1-43.

97 Hollós, L. 1902: Adatok a Kaukázus gombáinak ismeretéhez (in Hungarian). Növénytani Közlemények 1: 147-155.

98 Hollós, L. 1905: Beiträge zur Kenntnis der Pilzflora im Kaukasus (in German). Mathematische und naturwissenschaftliche berichte aus Ungarn 20: 315-325.

99 Jaczewski, A. 1893: Catalogue des Champignons recueillis en Russie en 1892 à Rylkowo, gouvernement de Smolensk (in French). Bulletin de la Société mycologique de France 9: 212-222.

100 Jaczewski, A.A. 1895: Catalogue of fungi of Smolensk Governorate, collected in 1892 and 1894 (in Russian). Bulletin de la Société impériale des naturalistes de Moscou 9: 128-130.

101 Jaczewski, A.A. 1907: Mycological flora of European and Asian Russia. Volume II. Slime moulds (in Russian). Typolithography of V. Richter: Moscow.

102 Javoronkowa 1914: Note préliminare concernant des observations sur la germination des spores de Didymium difforme Duby (in French). Bulletin de la Société Mycologique de France 30: 402-405.

103 Karpov, S.A., Novozhilov, Yu.K. \& Chistiakova, L.V. 2003: A comparative study of zoospore cytoskeleton in Symphytocarpus impexus, Arcyria cinerea and Lycogala epidendrum (Eumycetozoa). Protistology 3: 15-29.

104 Karsten, P.A. 1882: Enumeratio fungorum et Myxomycetum in Lapponia orientali aestate 1861 lectorum (impr. 1866) (in Latin). Notiser ur Sällskapets pro Fauna et Flora Fennica Förhandlingar 8: 193-224.

105 Karsten, P.A. 1906: Fungi in Transbaicalia, paucis exceptis prope fontes minerales Yamarovka, aestate ann. 1904 et 1905 a clar. P. Mikhno collecti (in Latin). Travaux de la Sous-Section Troïtzkossawsk-Kiaktha Section du pays d'Amour de la Société Imperiale Russe de Géographie 8: 60-64.

106 Kaukonen, M. 1996: Fungi of the former Kutsa Nature Reserve. Oulanka Reports 16: 69-72.

107 Kazey, A.S., Shirokikh, A.A. \& Shirokikh, I.G. 2015: Myxomycetes as a source of phytostimulating bacterias (in Russian). In: Mekhanizmy ustoychivosti i adaptatsii biologicheskikh sistem $\mathrm{k}$ prirodnym i tekhnogennym faktoram. Sbornik materialov Vserossiyskoy nauchnoy konferentsii (22-25 aprelya 2015 g.), 75-77. VESI: Kirov.

108 Khizhnyakova, A.S. \& Ronko, R.V. 2009: Myxomycetes of Nurgush Nature Reserve (in Russian). In: Nauchnye issledovaniya kak osnova okhrany prirodnykh kompleksov zapovednikov i zakaznikov. Materialy Vserossiyskoy nauchno-prakticheskoy konferentsii, 26 oktyabrya 2009 g., Vol. 1, 159-160. Staraya Vyatka: Kirov.

109 Killermann, S. 1943: Die höheren Pilze Sibiriens (in German). Annales Mycologici 41: 223-298.

110 Kochergina, A.V., Zemlianskaia, I.V. \& Novozhilov, Yu.K. 2005: Myxomycetes of the Elton Natural Park (in Russian with English abstract). In: Griby v prirodnykh i antropogennykh ekosistemakh. Trudy mezhdunarodnoy konferentsii, posvyashchennoy 100 -letiyu nachala raboty professora A.S. Bondartseva v Botanicheskom institute im. V.L. Komarova RAN, Sankt-Peterburg, 24-28 aprelya 2005 g., 300-302. Boston-spektr: St. Petersburg.

111 Kolomiytseva, D.Yu. \& Melkumov (Mel'kumov), G.M. 2015: New data on species diversity of myxomycetes of the forest communities of the reserve "Bryansky forest" to Bryansk region. In: Pavlov, S.I. (ed). Biological and ecological regional studies: global, Russian and regional problems. Proceedings of the 4th International Academic Conference, dedicated to the 115th anniversary of doctor of biological sciences, professor I.S. Sidoruk and doctor of agricultural sciences, professor P.A. Polozhentsev. 2015 December 7, Samara, Russian Federation, 64-67. Samara State Academy of Social Sciences and Humanities: Samara.

112 Kosheleva, A.P. 2000: Myxogasteromycetes and rare macromycetes of Lower Angara region (in Russian). In: Ekologiya i problemy zashchity okruzhayushchey sredy. Tezisy dokladov VII Vserossiyskoy studencheskoy nauchnoy konferentsii, 15-16. Krasnoyarsk.

113 Kosheleva, A.P. 2003a: Myxomycetes of Lower Angara Region (Taseyevsky district, Krasnoyarsk territory) (in Russian). In: Ekologiya i problemy zashchity okruzhayushchey sredy. Tezisy dokladov X Vserossiyskoy studencheskoy nauchnoy konferentsii, 19-20. Krasnoyarsk.

114 Kosheleva, A.P. 2003b: Myxomycetes of swamp complexes between $\mathrm{Ob}$ and Tom rivers (Tomsk region, Western Siberia) (in Russian). In: Bianki, A.M. (ed). Anichkovskiy vestnik, No 33: Molodye biologi Sankt-Peterburga 300-letiyu goroda. Materialy konferentsii, 53-54. Saint Petersburg City Palace of Youth Creativity: St. Petersburg. 
115 Kosheleva, A.P. 2003c: Myxomycetes of swamp complexes between $\mathrm{Ob}$ and Tom rivers (Tomsk region, Western Siberia) (in Russian). In: Biologiya - nauka XXI veka. 7-aya Pushchinskaya shkola-konferentsiya molodykh uchenykh 14-18 aprelya 2003 g. Sbornik tezisov, 180. Pushchino.

116 Kosheleva, A.P. 2004a: Myxomycetes of Stolby Nature Reserve (Krasnoyarsk territory, Eastern Siberia) (in Russian). In: Mikologiya i algologiya - 2004. Materialy yubileynoy konferentsii, posvyashchennoy 85-letiyu kafedry mikologii i algologii MGU im. M. V. Lomonosova, 78. Moscow.

117 Kosheleva, A.P. 2004b: Myxomycetes species new for Western Siberia (in Russian with English abstract). Mikologiya i Fitopatologiya 38: 41-42.

118 Kosheleva, A.P. 2004c: On study of morphological features of plasmodium of Licea operculata (Wing.) Martin (class Myxomycetes) (in Russian). In: Proceedings of the 8th Conference of Young Botanists in St. Petersburg (17-21 May 2004), 67-68. St. Petersburg State University of Technology and Design: St. Petersburg.

119 Kosheleva, A.P. 2004d: Study of myxomycetes of Krasnoyarsk territory (in Russian). In: Biologiya - nauka XXI veka. 8-aya Pushchinskaya shkola-konferentsiya molodykh uchenykh 17-21 maya 2004 g. Sbornik tezisov, 209. Pushchino.

120 Kosheleva, A.P. 2006: Myxomycetes from the various phytocoenoses of "Stolby" Strict Nature Reserve (Eastern Sayan, Russia) (in Russian with English abstract). Mikologiya i Fitopatologiya 40: 278-284.

121 Kosheleva, A.P. 2007a: Myxomycete diversity of the steppe mountain sections in the State Reserve "Stolby" (Eastern Sayan, Russia). In: Kovalenko, A., Melnik, V., Vedenyapina, E. \& Zmitrovich, I. (eds). XV Congress of European Mycologists. Saint Petersburg, Russia, September 16-21, 2007. Abstracts, 128-129. TREEART LCC: St. Petersburg.

122 Kosheleva, A.P. 2007b: Myxomycetes of Stolby Nature Reserve (Eastern Sayan): taxonomical composition and ecology (in Russian). PhD thesis. V.L. Komarov BIN RAS: St. Petersburg.

123 Kosheleva, A.P. \& Novozhilov, Yu.K. 2005: Substrate (adaptive) complexes of myxomycetes in Stolby Nature Reserve (in Russian). In: Mnogoletnie nablyudeniya v OOPT. Istoriya, sovremennoe sostoyanie, perspektivy. Sbornik statey po materialam vserossiyskoy nauchno-prakticheskoy konferentsii, posvyashchennoy 80 -letiyu GPZ "Stolby", 14-17 sentyabrya 2005 g. g. Krasnoyarsk, 211-215. Klaretianum: Krasnoyarsk.
124 Kosheleva, A.P., Novozhilov, Yu.K. \& Schnittler, M. 2008 : Myxomycete diversity of the state reserve "Stolby" (south-eastern Siberia, Russia). Fungal Diversity 31: 45-62.

125 Kotelenets, N.N. \& Barsukova, T.N. 2003: Myxomycetes and myxomycetophilous beetles in Oksky State Biospheric Reserve (in Russian with English abstract). Mikologiya i Fitopatologiya 37: 50-53.

126 Kruglikov, S.A. 2007: Slime moulds (myxomycetes) of Bryansky Les Nature Reserve (in Russian). In: Kingdom Fungi: true fungi, slime molds and lichens of the Bryansk Forest Nature Reserve, 42-43. Bryansk.

127 Kurbatova, M.E. 2017: New findings of myxomycetes in Volga-Akhtuba Flood Plain Nature Park (in Russian). In: Petrov, V.I. (ed). Aktualnye problemy eksperimentalnoy i klinicheskoy meditsiny. Materialy 75-y otkrytoy nauchno-prakticheskoy konferentsii molodykh uchenykh i studentov VolgGMU s mezhdunarodnym uchastiem, 487-488. Volgograd State Medical University: Volgograd.

128 Kurbatova, M.E. 2018: Members of genus Echinostelium in myxomycete biota of Volgo-Akhtuba Flood Plain Nature Park (in Russian). In: Petrov, V.I. (ed). Aktualnye problemy eksperimentalnoy i klinicheskoy meditsiny. Materialy 76-y mezhdunarodnoy nauchno-prakticheskoy konferentsii molodykh uchenykh i studentov, 415-416. Volgograd State Medical University: Volgograd.

129 Kutafyeva, N.P. \& Kosheleva, A.P. 2005: Materials on the biota of macromycetes and myxomycetes of Sayano-Shushensky Biosphere State Reserve (in Russian). Novosti sistematiki nizshikh rastenii 39: 139-145.

130 Kuznetsov, E.A. \& Tarasov, K.L. 2008: Fungus-like protists (in Russian with English abstract). In: Tchesunov, A.V., Kaljakina, N.M. \& Bubnova, E.N. (eds). A catalogue of biota of the White Sea Biological Station of the Moscow State University, 127-138. KMK Scientific Press Ltd., Moscow.

131 Lado, C. 2005-2020: An on line nomenclatural information system of Eumycetozoa: http://www.nomen.eumycetozoa.com/ (Date of access: 01.06.2020).

132 Lavrov (Lawrow), N.N. 1927: Beiträge zur Schleimpilz-Flora Sibiriens. I Schleimpilze der Umgebung von Tomsk [Materials on flora of Siberian slime moulds: I. Slime moulds of the vicinity of city of Tomsk] (in Russian with German abstract). Izvestia Tomskogo otdeleniya Russkogo Botanicheskogo obshchestva 2: 1-12. 
133 Lavrov, N. 1929: Formae novae myxomycetum Sibiriae [The new forms of slime molds from Siberia] (in Latin and Russian). Animadversiones systematicae ex Herbario Universitatis Tomskensis 4-5: 3 .

134 Lavrov (Lawrow), N.N. 1931: Beiträge zur Schleimpilz-Flora Sibiriens. II Schleimpilze des nördlichen und zentralen Altai [Materials on Siberian flora of slime moulds: II. Slime moulds of Northern and Central Altay] (in Russian with German abstract). Izvestia Tomskogo otdeleniya Russkogo Botanicheskogo obshchestva 3: 84-100.

135 Lebedev, A.N. 2007: Some results of researching flora of Myxomycetes of the Tver region (in Russian with English abstract). Tver State University Bulletin. Series: Biology and Ecology 5: 138-140.

136 Lebedev, A.N. 2008: Myxomycetes of Tver region (in Russian). PhD thesis. N.V. Tsitsin Main Botanical Garden: Moscow.

137 Lebedev, A.N. 2012: Special features of Didymium iridis (Ditmar) Fr. distribution in Tver region (in Russian). In: Biologicheskaya rekultivatsiya i monitoring narushennykh zemel. Materialy IX Vserossiyskoy nauchnoy konferentsii s mezhdunarodnym uchastiem, Yekaterinburg, 20-25 avgusta 2012 g., 141-143. Ural University Publishing House: Yekaterinburg.

138 Lebedev, A.N. \& Gmoshinskiy (Gmoshinsky), V.I. 2012: Distribution of Lycogala conicum Pers. (Myxomycetes) in Russia (in Russian with English abstract). Yaroslavl Pedagogical Bulletin 3: 115-117.

139 Lebedev, A.N. \& Notov, A.A. 2009: An annotated checklist of the slime moulds from the Botanical Garden of Tver State University (in Russian with English abstract). Tver State University Bulletin. Series: Biology and Ecology 13: 186-192.

140 Lebedev, A.N. \& Sinetenkova, M.A. 2013: New myxomycete species of Pskov region (in Russian). In: SymBioS Russia 2013. Abstracts of VI Russian Congress of Young Biologists with international participation. Proceedings of Educational School "New Concepts on Topical Problems in Biology and Medicine", 203-204. Asprint: Irkutsk.

141 Lebedev, A.N., Gmoshinskiy, V.I. \& Buchtoyarova, N.Yu. 2017: New data on Myxomycetes diversity in Central Forest Nature Reserve (Nelidovo area, Tver region) (in Russian with English abstract). Tver State University Bulletin. Series: Biology and Ecology 1: 217-236.
142 Lebedev, A.N., Notov, A.A. \& Korobkov, A.G. 2008 : Myxomycetes of the Udomel'skij district of the Tver region (in Russian with English abstract). Tver State University Bulletin. Series: Biology and Ecology 8: 136-142.

143 Lebedeva, L.A. 1933: Fungi and myxomycetes of Soviet Karelia (in Russian). Acta Instituti botanici Academiae Scientiarum URSS. Ser. 2, Fasc. 1: 329-331.

144 Leontyev, D.V. 2005: New data on myxomycetes in Cape Martyan Nature Reserve and Yalta Mountain-Forest Nature Reserve (in Russian). In: Zapovedniki Kryma: zapovednoe delo, bioraznoobrazie, ekoobrazovanie. Materialy III nauchnoy konferentsii (22 aprelya 2005 goda, Simferopol, Krym). Chast I. Geografiya. Zapovednoe delo. Botanika. Lesovedenie, 221-224. Simferopol.

145 Leontyev, D.V. 2010: Myxomycetes from the genera Stemonitis, Stemonitopsis and Stemonaria in Ukraine: identification and distribution (in Russian with English abstract). Mikologiya i Fitopatologiya 44: 398-409.

146 Leontyev, D.V. \& Fefelov, K.A. 2009: Tubulifera applanata. A new myxomycete species from Eastern Europe and Northern Asia. Boletín de la Sociedad Micológica de Madrid 33: 115-127.

147 Leontyev, D.V., Kochergina, A.V. \& Morozova, I.I. 2008 : Myxomycetes (in Russian). In: Popov, E.S. \& Rebriev, Yu.A (eds). IX Rabochee soveshchanie Komissii po izucheniyu makromitsetov (Vyoshenskaya, 4-10 oktyabrya 2006 g.). Annotirovannye spiski vidov gribov i miksomitsetov. Sbornik statey, 13-17. Rostov-on-Don.

148 Leontyev, D.V., McHugh, R., Fefelov, K.A. \& Kochergina, A.V. 2011: New and rare Myxomycetes of Ukraine. 2. Southwest Crimea. Nova Hedwigia 92: 245-256.

149 Leontyev, D.V., Schnittler, M., Moreno, G., Stephenson, S.L., Mitchell, D.W. \& Rojas, C. 2014: The genus Alwisia (Myxomycetes) revalidated, with two species new to science. Mycologia 106: 936-948.

150 Léveillé J.H. 1842: Observations médicales et énumération des plantes recueillies en Tauride (in French). In: Demidoff, A. (ed). Voyage dans la Russie méridionale et la Crimée, par la Hongrie, la Valachie et la Moldavie, Vol. 2, 33-232. Ernest Bourdin et Cie, Paris.

151 Loeselius, J. 1654: Plantae in Borussia sponte nascentes (in Latin). Typis Paschalii Mensenii: Regiomonti.

152 Loeselius, J. 1703: Flora Prussica, sive plantae in regno Prussiae sponte nascentes (in Latin). Sumptibus typographiae Georgianae: Regiomonti. 
153 Luptakova, A.D. 2018a: Data on the biota of Myxomycetes in the bottomland forests of Sok river in Samara region (in Russian with English abstract). In: Belanova, A.P. (ed). Perspektivy razvitiya i problemy sovremennoy botaniki. Materialy IV (VI) Vserossiyskoy molodezhnoy konferentsii s uchastiem inostrannykh uchenykh, 8-12 oktyabrya 2018 goda, g. Novosibirsk, 147-149. Akademizdat: Novosibirsk.

154 Luptakova, A.D. 2018b: Myxomycetes of Samara region (in Russian). In: Aleshkovskiy, I.A., Andriyanov, A.V. \& Antipov, E.A. (eds). International scientific conference of students and young scientists "Lomonosov-2018", 1-2. MAKS Press: Moscow.

155 Luptakova, A.D. 2019: Myxomycetes of Krasnosamarsky woodland in Samara region (in Russian). In: Aleshkovskiy, I.A., Andriyanov, A.V. \& Antipov, E.A. (eds). International scientific conference of students and young scientists "Lomonosov-2019", 1-2. MAKS Press: Moscow.

156 Luptakova, A.D. \& Korchikov, E.S. 2017: About the study of the myxomycetes of Krasnosamarsky woodland (in Russian with English abstract). In: Vasilyev, A.V. (ed). YOUNG ELPIT 2017. International Innovative Forum of Young Scientists in Framework of the VI International Environmental Congress (VIII International Scientific-Technical Conference) "Ecology and Life Protection of Industrial-Transport Complexes” ELPIT 2017 (Samara Togliatti, Russia, 20-24 September, 2017). Proceedings of the scientific reports, 176-181. Publishing House of Samara Scientific Centre: Samara.

157 Maniakina, M.V., Ostapenko, N.V. \& Zemlianskaia, I.V. 2005: Myxomycetes of the Sherbakov Natural Park (in Russian with English abstract). In: Griby v prirodnykh i antropogennykh ekosistemakh. Trudy mezhdunarodnoy konferentsii, posvyashchennoy 100 -letiyu nachala raboty professora A.S. Bondartseva v Botanicheskom institute im. V.L. Komarova RAN, Sankt-Peterburg, 24-28 aprelya 2005 g., 380-382. Boston-spektr: St. Petersburg.

158 Martius, H. 1817: Prodromus Florae Mosquensis (in Latin). Lipsiae.

159 Matveev, A.V. \& Gmoshinskiy, V.I. 2012: Myxomycetes of botanical gardens of Moscow (in Russian). In: Dyakov, Yu.T. (ed). Sovremennaya mikologiya v Rossii. Tom 3. Materialy 3-go Syezda mikologov Rossii, 133-134. National Academy of Mycology: Moscow.

160 Matveev, A.V. \& Gmoshinskiy (Gmoschinskiy), V.I. 2013: Myxomycetes of some botanical gardens of Moscow (in Russian). In: Terekhova, V.A. (ed). Bioindication in the
Ecological Assessment of Soils and Related Habitats. Book of Abstracts of the International Conference, Moscow, 4-6 February 2013, 140. BINOM. Laboratoriya znaniy: Moscow.

161 Matveev, A.V. \& Gmoshinskiy, V.I. 2015: First data on myxomycetes of the Botanical Garden of the Komarov Botanical Institute of RAS in moist chamber cultures (in Russian). In: Proceedings of III (XI) International Botanical Conference of Young Scientists in Saint-Petersburg 4-9 October 2015, 35-36. Komarov Botanical Institute of the Russian Academy of Sciences: St. Petersburg.

162 Matveev, A.V. \& Gmoshinskiy, V.I. 2017: First data on myxomycetes of Dagestan (in Russian). Trudy gosudarstvennogo prirodnogo zapovednika Dagestanskiy 13: 20-27.

163 Matveev, A.V., Gmoshinskiy, V.I., Botyakov, V.N. \& Novozhilov, Yu.K. 2018a: First records of Physarella oblonga (Myxomycetes) in Russia (in Russian with English abstract). Bulletin of Moscow Society of Naturalists. Biological series 123: 66-77.

164 Matveev, A.V., Gmoshinsky, V.I., Prokhorov, V.P. \& Kazantseva, E.S. 2018b: The myxomycetes from botanical gardens of Moscow: N.V. Tsitsin Botanical Garden of the Russian Academy of Sciences and the Botanical Garden of Moscow State University. Mikologiya i Fitopatologiya 52: 106-111.

165 Matveev, A.V., Lebedev, A.N. \& Gmoshinskiy, V.I. 2014: The first data on myxomycetes of the Botanical Garden of Tver State University obtained using moist chamber cultures (in Russian). In: Problems and perspectives of the plant world investigations. International scientific and practical conference of the young scientists. Book of abstracts. Yalta, May 13-16th 2014, 119. Yalta.

166 Matveev, A.V., Lebedev, A.N. \& Gmoshinskiy, V.I. 2018c: Results of long-term research of myxomycetes biota in the Tver State University Botanical Garden (in Russian with English abstract). Mikologiya i Fitopatologiya 52: 112-119.

167 Melkumov, G.M. 2017: Species composition of plasmodial slime moulds (Myxomycetes) of Novousmansky district of Voronezh region (in Russian). In: Sovremennaya lesnaya nauka: problemy i perspektivy. Materialy Vserossiyskoy nauchno-prakticheskoy konferentsii 20-22 dekabrya 2017, 173-178. Istoki: Voronezh.

168 Melkumov, G.M. 2019: New data on myxomycetes (Myxomycota) of Orlovskoye Polesye National Park (in Russian). In: Voskresenskaya, O.L. (ed). Sovremennye problemy meditsiny i estestvennykh nauk. Sbornik statey Mezhdunarodnoy nauchnoy konferentsii, Yoshkar-Ola, 15-19 
aprelya 2019 g., Vol. 8, 145-146. Mari State University: Yoshkar-Ola.

169 Melkumov, G.M. 2020: Hidden diversity of plasmodial myxomycetes (Myxomycetes) of Voronezh region (in Russian). In: Agafonov, V.A. (ed). Problemy botaniki: istoriya i sovremennost. Materialy Mezhdunarodnoy nauchnoy konferentsii, posvyashchennoy 130 -letiyu so dnya rozhdeniya prof. B.M. Kozo-Polyanskogo, 80-letiyu so dnya rozhdeniya prof. K.F. Khmeleva, IX nauchnogo soveshchaniya "Flora Sredney Rossii" (Voronezh, 3-7 fevralya 2020 g.), 245-248. Tsifrovaya poligrafiya: Voronezh.

170 Melkumov, G.M. \& Kolomiytseva, D.Yu. 2016: First data on myxomycetes of Voronezh region obtained using moist chamber cultures (in Russian with English abstract). Proceedings of Voronezh State University. Series: Chemistry. Biology. Pharmacy 3: 85-88.

171 Melkumov, G.M. \& Plotnikova, K.A. 2018: Substrate specialization of plasmodial slime molds (Myxomycetes) [in] Natural reserve of regional importance "Voronezh upland oak forest" (in Russian with English abstract). In: Morkovina, S.S. (ed). Ekologicheskie i biologicheskie osnovy povysheniya produktivnosti i ustoychivosti prirodnykh $\mathrm{i}$ iskusstvenno vozobnovlennykh lesnykh ekosistem. Materialy mezhdunarodnoy nauchno-prakticheskoy konferentsii, posvyashchennoy 100-letiyu vysshego lesnogo obrazovaniya v g. Voronezh i TsChR Rossii, Vol. 1, 165-173. Voronezh State University of Forestry and Technologies named after G.F. Morozov: Voronezh.

172 Mishulin, A.A. 2018: New data on myxomycete biota of Vladimir region (in Russian). In: Aleshkovskiy, I.A., Andriyanov, A.V. \& Antipov, E.A. (eds). International scientific conference of students and young scientists "Lomonosov-2018", 1-2. MAKS Press: Moscow.

173 Mishulin, A.A., Blinova, A.V. \& Gushchina, S.E. 2018: Myxomycetes and fungi of the forest seed reserve "Sudogodsky" (in Russian with English abstract). In: Gracheva, E.P. (ed). Problemy ekologicheskogo obrazovaniya $v$ XXI veke. Trudy II Mezhdunarodnoy nauchnoy konferentsii (ochno-zaochnoy), 6-12. Arkaim: Vladimir.

174 Moreno, G., Lopez-Villalba, A., Castillo, A., Romanenko, K.O. \& Leontyev, D.V. 2017: Notes on some myxomycetes from Crimea (Ukraine). Mycotaxon 132: 649-663.

175 Mosolov, N.A. 1906: List of fungi found in Podolsky uyezd. Second expanded edition (in Russian). Typolithography of I.N. Kushnerev and Co partnership: Moscow.

176 Murashkinskiy, K.E. 1911: Descriptive catalogue of the Nat- ural History Museum of Nizhny Novgorod Governorate Zemstvo. Issue 3. Botanical department: catalogue of the fungal herbarium (in Russian). Typography of $V$. Royskiy and I. Karneev: Nizhniy Novgorod.

177 Naoumoff, M.N. 1914: Matériaux pour la flore mycologique da la Russie, Fungi ussurienses I (in French). Bulletin de la Société Mycologique de France 30: 64-83.

178 Naumenko, Y.V., Gorbunova, I.A., Vlasenko, V.A. \& Vlasenko, A.V. : Mycologic herbarium of Central Siberian Botanical Garden SB RAS. History and prospects of development (in Russian). Rastitelnyy mir Aziatskoy Rossii 29: 100-104.

179 Notov, A.A. \& Lebedev, A.N. 2009: Myxomycetes in Zavidovo National Park (in Russian). In: Fertikov, V.I. (ed). Zavidovo National Park. 80 years (1929-2009). Anniversary scientific readings, Vol. 7, 208-215. Delovoy mir, Moscow.

180 Novozhilov, Yu.K. 1980a: Mixomycetes occurring in the Central State Forest Reservation ([and] Leningrad region) (in Russian with English abstract). Mikologiya i Fitopatologiya 14: 198-201.

181 Novozhilov, Yu.K. 1980b: New species of Myxomycetes in the U.S.S.R. (in Russian). Mikologiya i Fitopatologiya 14: 314-318.

182 Novozhilov, Yu.K. 1981a: Myxomycetes of Ladoga-Ilmen floristic region (in Russian). PhD thesis. V.L. Komarov BIN RAS: Leningrad.

183 Novozhilov, Yu.K. 1981b: Polytomic figure keys for identifying myxomycetes genera and species of Leningrad region (in Russian). Mikologiya i Fitopatologiya 15: 6-16.

184 Novozhilov, Yu.K. 1983: Myxomycete herbarium of the Komarov Botanical Institute of the USSR Academy of Sciences (in Russian). Mikologiya i Fitopatologiya 17: 449-451.

185 Novozhilov, Yu.K. 1984: Mixomycetes occurring in Kursk region and Aliokhin State Central Chernozem Biosphere Reservation (in Russian). Mikologiya i Fitopatologiya 18: 26-28.

186 Novozhilov, Yu.K. 1985a: Mixomycetes of the U.S.S.R. I. Genus Echinostelium D By. (in Russian). Mikologiya i Fitopatologiya 19: 124-128.

187 Novozhilov, Yu.K. 1985b: Mixomycetes of the U.S.S.R. II. Genus Licea Schrad. (in Russian). Mikologiya i Fitopatologiуа 19: 290-297. 
188 Novozhilov (Novogilov), Ju.K. 1986a: De myxomycetis nivalibus regionis Leningradensis notula [Nivicolous myxomycetes of Leningrad region] (in Russian). Novosti sistematiki nizshikh rastenii 23: 146-149.

189 Novozhilov (Novogilov), Ju.K. 1986b: De myxomycetis paeninsulae Czukotka notula. I. [On myxomycetes of Chukotka. I.] (in Russian). Novosti sistematiki nizshikh rastenii 23: $143-146$.

190 Novozhilov, Yu.K. 1986c: Mixomycetes of the U.S.S.R. III. Genus Macbrideola H.C. Gilbert (in Russian). Mikologiya i Fitopatologiya 20: 102-105.

191 Novozhilov, Ju.K. 1987: De Myxomycetibus e reservato Altaiensi notula [Myxomycetes of Altai Nature Reserve] (in Russian). Novosti sistematiki nizshikh rastenii 24: 113-115.

192 Novozhilov, Yu.K. 1988: Epiphytic myxomycetes in some regions of the USSR. Analysis of their substrate and habitat distribution (in Russian). Mikologiya i Fitopatologiya 22: 301-307.

193 Novozhilov, Yu.K. 1993a: Definitorium Fungorum Rossiae. Divisio Myxomycota. Fasc. 1. Classis Myxomycetes (in Russian). Nauka: Petropolis.

194 Novozhilov, Yu.K. 1993b: Myxomycetes of Russia. I. Survey of the genus Cribraria Pers (in Russian with English abstract). Mikologiya i Fitopatologiya 27: 39-47.

195 Novozhilov, Yu.K. 1999a: Myxomycetes of the Leningrad region (in Russian with English abstract). In: Balashova, N.B. \& Zavarzin, A.A. (eds). Biodiversity of the Leningrad region. Ser. 6. (Algae. Fungi. Lichens. Bryophytes. Invertebrates. Fish and fish-like). Collection of articles, Vol. 2, 197-204. St. Petersburg University Publishing House, St. Petersburg.

196 Novozhilov, Yu.K. 1999b: Myxomycota (in Russian). In: Karatygin, I.V., Nezdoiminogo, E.L., Novozhilov, Yu.K. \& Zhurbenko, M.P. (eds). Russian Arctic Fungi. Check-list, 19-30. St. Petersburg State Chemical Pharmaceutical Academy, St. Petersburg.

197 Novozhilov, Yu.K. 2000: Fungi and Slime moulds (in Russian and English). In: Tzvelev, N.N. (ed). Red Data Book of Nature of the Leningrad region. Vol. 2. Plants and Fungi, 495-652. World \& Family, St. Petersburg.

198 Novozhilov, Yu.K. 2005: Myxomycetes (class Myxomycetes) of Russia: taxonomical composition, ecology, and geography (in Russian). Doctoral dissertation. V.L. Komarov BIN RAS: St. Petersburg.
199 Novozhilov, Yu.K. 2007: Slime moulds (in Russian). In: Tsvelev, N.N. (ed). Environment and biological diversity of Berezovye islands archipelago (the Gulf of Finland), 270-274. St. Petersburg.

200 Novozhilov, Yu.K. 2007-2020: The Myxomycetes Collection at the V.L. Komarov Botanical Institute, St. Petersburg. Occurrence dataset: https://doi.org/10.15468/i83r9k (Date of access: 01.06.2020).

201 Novozhilov, Yu.K. \& Fefelov, K.A. 2001: An annotated checklist of the myxomycetes of Sverdlovsk region, West Siberian lowland, Russia. Mikologiya i Fitopatologiya 35: 41-52.

202 Novozhilov, Yu.K. \& Kosheleva, A.P. 2006: Myxomycetes (class Myxomycetes) of Orenburg State Nature Reserve (in Russian). In: Chibilyov, A.A. (ed). Stepi Severnoy Evrazii. Materialy IV mezhdunarodnogo simpoziuma, 521-524. Gazprompechat: Orenburg.

203 Novozhilov, Ju.K. \& Krussanova, Z.G. 1989: Myxomycetes reservati lazoviani (Prov. Primorskensis) [Myxomycetes from Lazovski reserve (Primorsky territory)] (in Russian). Novosti sistematiki nizshikh rastenii 26: 69-72.

204 Novozhilov, Yu.K. \& Lebedev, A.N. 2006: Annotated check-list of lignicolous myxomycetes from Tver' oblast' (in Russian with English abstract). Mikologiya i Fitopatologiya 40: 236-245.

205 Novozhilov, Yu.K. \& Schnittler, M. 1996: Nivicole Myxomycetes of the Khibine Mountains (Kola peninsula). Nordic Journal of Botany 16: 549-561.

206 Novozhilov, Yu.K. \& Schnittler, M. 2000: A new coprophilous species of Perichaena (Myxomycetes) from the Russian Arctic (the Taimyr Peninsula and the Chukchi Peninsula). Karstenia 40: 117-122.

207 Novozhilov, Yu.K. \& Zemlyanskaya, I.V. 2006: A new species of Didymium (Myxomycetes) with reticulate spores. Mycotaxon 96: 147-150.

208 Novozhilov, Yu.K., Hooff, H. \& Jagers, M. 2015: Trichioides iridescens, a new genus and new species (incertae sedis in Myxomycetes). Mycological Progress 14: 1-7.

209 Novozhilov, Yu.K., Okun, M.V., Erastova, D.A., Shchepin, O.N., Zemlyanskaya, I.V., García-Carvajal, E. \& Schnittler, M. 2013a: Description, culture and phylogenetic position of a new xerotolerant species of Physarum. Mycologia 105: 1535-1546. 
210 Novozhilov, Yu.K., Schnittler, M. \& Stephenson, S. 1998: The myxomycetes of Russian subarctic and arctic areas. Mikologiya i Fitopatologiya 32: 18-29.

211 Novozhilov, Yu.K., Schnittler, M. \& Stephenson, S.L. 1999: Myxomycetes of the Taimyr Peninsula (North-Central Siberia). Karstenia 39: 77-97.

212 Novozhilov, Yu.K., Schnittler, M., Zemlianskaia, I.V. \& Fefelov, K.A. 2000: Biodiversity of plasmodial slime moulds (Myxogastria): measurement and interpretation. Protistology 1: 161-178.

213 Novozhilov, Yu.K., Schnittler, M. \& Zemlianskaia, I.V. 2005a: Synecology of myxomycetes in desert of the northwestern Caspian lowland (in Russian with English abstract). Mikologiya i Fitopatologiya 39: 40-52.

214 Novozhilov, Yu.K., Schnittler, M., Vlasenko, A.V. \& Fefelov, K.A. 2009: Myxomycete diversity of the Chuyskaya depression (Altay, Russia). Mikologiya i Fitopatologiya 43: 522-534.

215 Novozhilov, Yu.K., Schnittler, M., Vlasenko, A.V. \& Fefelov, K.A. 2010: Myxomycete diversity of the Altay Mountains (southwestern Siberia, Russia). Mycotaxon 111: 91-94.

216 Novozhilov, Yu.K., Schnittler, M., Fiore-Donno, A.M., Meyer, M., Erastova, D.A., Heinrich, E. \& Schepin, O.N. 2011: Taxonomical and ecological diversity of nivicolous myxomycetes of the Teberda Nature Reserve (Karachay-Cherkessia, Russia). In: The 7th International Congress on Systematics and Ecology of Myxomycetes (ICSEM7). Proceedings, 7-8. Recife, Brazil.

217 Novozhilov, Yu.K., Schnittler, M., Erastova, D.A., Okun, M.V., Schepin, O.N. \& Heinrich, E. 2013b: Diversity of nivicolous myxomycetes of the Teberda State Biosphere Reserve (Northwestern Caucasus, Russia). Fungal Diversity 59: 109-130.

218 Novozhilov, Yu.K., Schnittler, M., Erastova, D.A. \& Shchepin, O.N. 2017a: Myxomycetes of the Sikhote-Alin State Nature Biosphere Reserve (Far East, Russia). Nova Hedwigia 104: 183-209.

219 Novozhilov, Yu.K., Shchepin, O.N. \& Schnittler, M. 2017b: A preliminary report of the diversity of nivicolous myxomycetes from the Natural Park "Vulkany Kamchatki" (in Russian and English). In: Tokranov, A.M. (ed). Conservation of biodiversity of Kamchatka and coastal waters. Materials of the XVIII International scientific conference, dedicated to the 70th anniversary of P.A. Khomentovsky's birthday, Petropavlovsk-Kamchatsky, November 15-16, 2017, 371-378. Kamchatpress: Petropavlovsk-Kamchatskiy.
220 Novozhilov, Yu.K., Zemlyanskaia, I.V., Schnittler, M. \& Fefelov, K.A. 2003: An annotated checklist of the myxomycetes of the northwestern Caspian lowland. Mikologiya i Fitopatologiya 37: 53-65.

221 Novozhilov, Yu.K., Zemlianskaia, I.V. \& Schnittler, M. 2005b: Corticolous myxomycetes in deserts of the Northwestern Caspian lowland (in Russian with English abstract). Mikologiya i Fitopatologiya 39: 43-54.

222 Novozhilov, Yu.K., Zemlianskaia, I.V. \& Schnittler, M. 2005c: Myxomycetes of the Northwestern Caspian deserts (in Russian with English abstract). Novosti sistematiki nizshikh rastenii 38: 164-171.

223 Novozhilov, Yu.K., Zemlyanskaya, I.V., Schnittler, M. \& Stephenson, S.L. 2006: Myxomycete diversity and ecology in the arid regions of the Lower Volga River Basin (Russia). Fungal Diversity 23: 193-241.

224 Novozhilov, Yu.K., Zemlyanskaya, I.V., Schnittler, M. \& Stephenson, S.L. 2008: Two new species of Perichaena (Myxomycetes) from arid areas of Russia and Kazakhstan. Mycologia 100: 810-816.

225 Okun, M.V. 2013: Molecular phylogeny and taxonomical status of myxomycete species of the Physarum notabile complex (in Russian). PhD thesis. V.L. Komarov BIN RAS: St. Petersburg.

226 Okun, M. 2014: Molecular phylogeny and taxonomy of the Physarum notabile species complex (Myxomycetes).

PhD thesis. Universität Wien: Wien.

227 Pallas, P.S. 1771: Reise durch verschiedene Provinzen des Russischen Reichs. Erster Teil (in German). Kaiserliche Akademie der Wissenschaften: St. Petersburg.

228 Pallas, P.S. 1773: Travel to different provinces of the Russian Empire. Part I. (in Russian). Russian Academy of Science: St. Petersburg.

229 Pallas, P.S. 1788: Voyages de M. P. S. Pallas en différentes provinces de l'Empire de Russie, et dans l'Asie septentrionale. Tome premier (in French). Lagrange: Paris.

230 Pallas, P.S. 1794: Voyages du professeur Pallas, dans plusieurs provinces de l'Empire de Russie et dans l'Asie septentrionale. Tome premier (in French). Maradan: Paris.

231 Petrenko, I.A. 1978: Macro- and micromycetes of Yakutian forests (in Russian). Nauka: Novosibirsk.

232 Petrov, A.N. \& Matosova, E.A. 2010: Synantropic mycobiota of South Prebaicalia: Myxomycetes, Ascomycetes, Heterobasidiomycetes, Aphyllophorales, Gasteromycetes 
(in Russian with English abstract). The Bulletin of Irkutsk State University. Series: Biology and Ecology 3: 3-8.

233 Petrova, N.V. \& Gorbunova, N.A. 2001: Macromycetes of southern part of Western Siberia (in Russian). Publishing House of SB RAS: Novosibirsk.

234 Plotnikov, B.S. 2005: Systematic structure of myxomycete communities in gradient of technogenic polution (in Russian). In: Ekologiya: ot genov do ekosistem. Materialy konferentsii molodykh uchenykh, 25-29 aprelya 2005 g., 206-211. Akademkniga: Yekaterinburg.

235 Plotnikov, B.S. \& Fefelov, K.A. 2003: Taxonomical composition of myxomycetes in the city of Yekaterinburg (in Russian). In: Problemy globalnoy i regionalnoy ekologii. Materialy konferentsii molodykh uchenykh, 31 marta - 4 aprelya 2003 g., 185-186. Akademkniga: Yekaterinburg.

236 Plotnikov, B.S. \& Fefelov, K.A. 2009: Myxomycetes under the pollution gradient of a copper plant in southern taiga, the Middle Urals (in Russian with English abstract). Mikologiya i Fitopatologiya 43: 33-44.

237 Popov, E.S., Morozova, O.V., Kotkova, V.M., Novozhilov, Yu.K., Zhurbenko, M.P., Zmitrovich, I.V. \& Kovalenko, A.E. 2007: Preliminary list of fungi and myxomycetes of Leningrad region. TREEART LCC: St. Petersburg.

238 Prokhorov, V.P. \& Bodyagin, V.V. 2007: Ecology of aquatic hyphomycetes (in Russian with English abstract). Vestnik Moskovskogo universiteta. Seriya 16. Biologiya 1: 19-24.

239 Radkova, N.A. 1995: Myxomycetes of the alder forests of Khopyor Nature Reserve (in Russian). In: Problemy izucheniya i okhrany zapovednykh prirodnykh kompleksov. Materialy nauchnoy konferentsii, posvyashchennoy 60letiyu Khoperskogo zapovednika (pos. Varvarino, Voronezhskaya oblast, 21-25 avgusta 1995 g.), 45-46. Voronezh State University: Voronezh.

240 Rebhahn, M.-A., Schnittler, M. \& Liebermann, B. 1999: Taxonomic relevance of pigment patterns in Arcyria species (Trichiales, Myxomycetes) including Arcyodes incarnata. Nova Hedwigia 69: 415-427.

241 Romanenko, K.O. 2001: Corticolous and lignicolous myxomycetes of Crimean Nature Reserve (in Ukrainian). Uchenye zapiski Tavricheskogo natsionalnogo universiteta im. V.I. Vernadskogo. Seriya: Biologiya 14: 184-188.

242 Romanenko, E.A. 2002a: Species diversity of litter myxomycetes in Crimean Natural Reserve (Ukraine) (in Russian with English abstract). Mikologiya i Fitopatologiya 36: 51-58.
243 Romanenko, K.O. 2002b: Myxomycetes of the plant communities of the Crimean Natural Reserve (in Ukrainian with Russian and English abstract). Ukrainian Botanical Journal 59: 730-736.

244 Romanenko, K.O. 2006: Myxomycetes of Crimean Nature Reserve (in Ukrainian). PhD thesis. M.G. Kholodny Institute of Botany of the National Academy of Sciences of Ukraine: Kyiv.

245 Rosanoff, S. 1868: De l'influence de l'attraction terrestre sur la direction des plasmodia des myxomycètes (in French). Mémoires de la Société impériale des sciences naturelles de Cherbourg 14: 149-172.

246 Rostafiński, J. 1874: Śluzowce (Mycetozoa), Monografia, część pierwsza (in Polish). Pamiętnik Towarzystwa Nauk Ścisłych w Paryżu 5: 1-215.

247 Rostafiński, J. 1875: Śluzowce (Mycetozoa), Monografia, część druga (in Polish). Pamiętnik Towarzystwa Nauk Ścisłych w Paryżu 6: 217-432.

248 Rtishcheva, A.I. \& Belova, V.E. 1992: Myxomycetes and their role in forest phytocoenoses of Voronezh region (in Russian). In: The state and problems of the ecosystem of Usmansky pine forest. Proceedings of the Venevitinovo Educational and Research Biocenter of the Voronezh State University, Vol. 2, 156-160. Voronezh.

249 Rtishcheva, A.I. \& Rodionova, N.A. 2005: Myxomycetes and macromycetes of Khopersky Nature Reserve (in Russian). VGPU: Voronezh.

250 Saccardo, P.A. 1880: Fungorum extra-europaeorum pugillus (in Latin). Michelia 2: 136-149.

251 Sanamyan, K.E. 2006: Preliminary report on Myxomycetes of Kamchatka with short information about their systematic position (in Russian). In: Tokranov, A.M. (ed). Conservation of biodiversity of Kamchatka and coastal waters. Materials of VII International scientific conference, Petropavlovsk-Kamchatsky, November 28-29, 2006, 145-149. Petropavlovsk-Kamchatskiy.

252 Sarycheva, L.A. 1999: Fungi and myxomycetes of Galichiya Gora Nature Reserve (in Russian). Voronezh.

253 Schell, J. 1883: Materials for botanical geography of the Ufa and Orenburg Governorates (Cryptogamic plants) (in Russian). Trudy obshchestva yestestvoispytateley pri Imperatorskom Kazanskom Universitete 12: 1-93. 
254 Schnittler, M. \& Novozhilov, Yu.K. 1996: The myxomycetes of boreal woodlands in Russian northern Karelia: a preliminary report. Karstenia 36: 19-40.

255 Schnittler, M., Erastova, D.A., Shchepin, O.N., Heinrich, E. \& Novozhilov, Yu.K. 2015: Four years in the Caucasus - observations on the ecology of nivicolous myxomycetes. Fungal Ecology 14: 105-115.

256 Schnittler M., Shchepin O.N., Dagamac N.H.A., Borg Dahl, M. \& Novozhilov Y.K. 2017: Barcoding myxomycetes with molecular markers: challenges and opportunities. Nova Hedwigia 104: 323-341.

257 Shchepin, O.N., Dagamac, N.H., Sanchez, O.M., Novozhilov, Yu.K., Schnittler, M. \& Zemlyanskaya, I.V. 2017: DNA barcoding as a tool for identification of plasmodia and sclerotia of myxomycetes (Myxogastria) appearing in moist chamber cultures. Mycosphere 8: 1904-1913.

258 Shchepin, O., Novozhilov, Yu. \& Schnittler, M. 2014: Nivicolous myxomycetes in agar culture: some results and open problems. Protistology 8: 53-61.

259 Shchepin, O.N., Novozhilov, Yu.K. \& Schnittler, M. 2016: Disentangling the taxonomic structure of the Lepidoderma chailletii-carestianum species complex (Myxogastria, Amoebozoa): genetic and morphological aspects. Protistology 10: 119-129.

260 Shchepin, O.N., Schnittler, M., Dagamac, N.H.A., Leontyev, D.V. \& Novozhilov, Yu.K. 2019a: Unexplored diversity of microscopic myxomycetes: evidence from environmental DNA. Plant Ecology and Evolution 152: 499-506.

261 Shchepin O.N., Schnittler M., Erastova D.A., Prikhodko I.S., Borg Dahl, M., Azarov D.V., Chernyaeva E.N. \& Novozhilov Yu.K. 2019b: Community of dark-spored myxomycetes in ground litter and soil of taiga forest (Nizhne-Svirskiy Reserve, Russia) revealed by DNA metabarcoding. Fungal Ecology 39: 80-93.

262 Shiliakow, N. 1889: Zur Myxomyceten-Flora des Gouvernements Kazan [Notes on myxomycete findings in Kazan Governorate] (in Russian with German abstract). Scripta Botanica Horti Universitatis Imperialis Petropolitanae 2: 25-34.

263 Shirokikh, A.A. 2009: Preliminary data on myxomycetes of Nurgush Nature Reserve (in Russian). In: Nauchnye issledovaniya kak osnova okhrany prirodnykh kompleksov zapovednikov i zakaznikov. Materialy Vserossiyskoy nauchno-prakticheskoy konferentsii, 26 oktyabrya 2009 g., Vol. 1, 178-183. Staraya Vyatka: Kirov.
264 Shirokikh, A.A. 2011: Lignicolous myxomycetes of Nurgush Nature Reserve (in Russian). In: Tselishcheva, L.G. (ed). Proceedings of the Nurgush State Nature Reserve, Vol. 1, 182-187. Staraya Vyatka, Kirov.

265 Shirokikh, A.A. 2013a: Myxomycetes of Nurgush Nature Reserve (in Russian). Theoretical and Applied Ecology 2: 136-142.

266 Shirokikh, A.A. 2013b: Study of myxomycete species composition of substrate complexes in Nurgush Nature Reserve (in Russian). In: Modern problems in botanical and mycological research. Proceedings of the II international scientific and practical conference, Minsk, November 12-14, 2013, 123-125. Publishing Centre of the Belarusian State University: Minsk.

267 Shirokikh, A.A. 2017: More on species and genetic diversity of myxomycetes (case study of Nurgush Nature Reserve) (in Russian). In: Tselishcheva, L.G. (ed). Proceedings of the Nurgush State Nature Reserve, Vol. 4, 148-153. Staraya Vyatka, Kirov.

268 Shirokikh, A.A. 2018: Myxomycetes of Nurgush Nature Reserve (in Russian). Staraya Vyatka: Kirov.

269 Shirokikh, A.A. \& Berezina, Yu.S. 2017: Fungus-like protists as a component of forest ecosystems (in Russian). In: Savinykh, N.P., Perestoronina, O.N., Domnina, E.A., Shabalkina, S.V. \& Shakleina, M.N. (eds). Sokhranenie lesnykh ekosistem: problemy i puti ikh resheniya. Materialy Vserossiyskoy nauchno-prakticheskoy konferentsii (g. Kirov, 15-19 maya 2017 g.), 275-279. Raduga-Press: Kirov.

270 Shirokikh, A.A. \& Panyukova, E.V. 2019: Mixomicotes (Myxomycetes) on the plain part of the Pechoroilychsky reserve of the Komi Republic (in Russian with English abstract). In: Petrenko, D.B. (ed). Aktualnye problemy biologicheskoy i khimicheskoy ekologii. Sbornik materialov VI Mezhdunarodnoy nauchno-prakticheskoy konferentsii (g. Mytishchi, 26-28 fevralya 2019 goda), 26-28. Moscow Region State University: Moscow.

271 Shirokikh, A.A. \& Shikhalyova, O.P. 2011: Results of track surveys of lignicolous myxomycetes of Nurgush Nature Reserve (in Russian). In: Biologicheskiy monitoring prirodno-tekhnogennykh sistem. Sbornik materialov Vserossiyskoy nauchno-prakticheskoy konferentsii s mezhdunarodnym uchastiem v 2 chastyakh. Chast 2. (g. Kirov, 29-30 noyabrya 2011 g.), Vol. 2, 188-190. Loban: Kirov.

272 Shirokikh, A.A. \& Shirokikh, I.G. 2010: Diversity of myxomycetes in forest park zone of the city of Kirov (in Russian). Immunopathology, Allergology, Infectology 1: 54-55. 
273 Shirokikh, A.A. \& Shirokikh, I.G. 2014: Bacteria in fruit bodies of myxomycetes (in Russian). In: Netrusov, A.I. \& Kolotilova, N.N. (eds). Sovremennye problemy fiziologii, ekologii i biotekhnologii mikroorganizmov. Vserossiyskiy simpozium s mezhdunarodnym uchastiem. Moskva, MGU imeni M.V. Lomonosova. Biologicheskiy fakultet. 24-27 dekabrya 2014 g.: Materialy, 255. MAKS Press: Moscow.

274 Shirokikh, A.A. \& Shirokikh, I.G. 2018: Occurrence of Myxogasteromycetes in parks of the city of Kirov (in Russian). In: Materialy Vserossiyskoy konferentsii s mezhdunarodnym uchastiem, "Mikologiya i algologiya Rossii. XX - XXI vek: smena paradigm”, posvyashchennoy 100-letiyu kafedry mikologii i algologii biologicheskogo fakulteta MGU imeni M.V. Lomonosova, 110-letiyu so dnya rozhdeniya professora Mikhaila Vladimirovicha Gorlenko, pamyati professora Yuriya Tarichanovicha Dyakova, 242-243. Pero: Moscow.

275 Shirokikh, A.A. \& Shirokikh, I.G. 2019a: Biodiversity of slime molds on reference sites of the middle and south taiga of the Kirov region (in Russian with English abstract). In: Savinykh, N.P. (ed). Sokhranenie lesnykh ekosistem: problemy i puti ikh resheniya. Materialy II Mezhdunarodnoy nauchno-prakticheskoy konferentsii (g. Kirov, 27-31 maya 2019 g.), 315-319. Vyatka State University: Kirov.

276 Shirokikh, A.A. \& Shirokikh, I.G. 2019b: Methodical approaches to the study of slime moulds in urban ecosystems (in Russian). In: Ashikhmina, T.Ya. (ed). Transformatsiya ekosistem pod vozdeystviem prirodnykh i antropogennykh faktorov. Materialy mezhdunarodnoy nauchnoy konferentsii (g. Kirov, 16-18 aprelya 2019 g.), 132-135. Vyatka State University: Kirov.

277 Shirokikh, A.A., Poplyvanov, D.V. \& Kremsal, A.V. 2017: Myxomycete findings in the city of Kirov (in Russian). In: Ashikhmina, T.Ya. (ed). Biodiagnostika sostoyaniya prirodnykh i prirodno-tekhnogennykh sistem. Materialy XV Vserossiyskoy nauchno-prakticheskoy konferentsii c mezhdunarodnym uchastiem. Kniga 2 (g. Kirov, 4-6 dekabrya 2017 g.), 52-57. Vyatka State University: Kirov.

278 Sizova, T.P. \& Titova, Yu.A. 1985: Slime fungi on the territory of Zvenigorodskaya Biological Station (Biological Department of the Moscow State University) (in Russian with English abstract). Bulletin of Moscow Society of Naturalists. Biological series 90: 113-117.

279 Sizova, T.P., Titova, J.A. \& Daracov, O.B. 1983: Species nova e genere Trichia (Myxomycetes) [A new species of genus Trichia (Myxomycetes)] (in Russian). Novosti sistematiki nizshikh rastenii 20: 121-122.
280 Skvortsova, A.V. 2017: New data on myxomycetes of Shcherbakovsky Nature Park (in Russian). In: Petrov, V.I. (ed). Aktualnye problemy eksperimentalnoy i klinicheskoy meditsiny. Materialy 75-y otkrytoy nauchno-prakticheskoy konferentsii molodykh uchenykh i studentov VolgGMU s mezhdunarodnym uchastiem, 501-502. Volgograd State Medical University: Volgograd.

281 Smolnyakova, Yu.A. 2013: Myxomycetes of the flood plain oak woods in interfluve of Volga and Akhtuba (in Russian). In: XVIII Regionalnaya konferentsiya molodykh issledovateley Volgogradskoy oblasti, Volgograd, 5-8 noyabrya 2013 g. Sbornik nauchnykh materialov. Napravlenie 11 "Biologiya i geografiya", 40-42. Peremena: Volgograd.

282 Smolnyakova, Yu.A. 2017: Problems and prospects of inclusion of slime-molds (Myxomycetes) into the regional Red Lists (in Russian). In: Vedenie regionalnykh Krasnykh knig: dostizheniya, problemy i perspektivy. Sbornik materialov III Vserossiyskoy nauchno-prakticheskoy konferentsii s mezhdunarodnym uchastiem, Volgograd, 25-28 oktyabrya 2017 g., 113-115. Kruton: Volgograd.

283 Smolnyakova, Yu.A. \& Zemlyanskaya, I.V. 2015: Myxomycetes in oak forests of the Volga-Akhtuba floodplain Nature Park (in Russian). In: Proceedings of III (XI) International Botanical Conference of Young Scientists in Saint-Petersburg 4-9 October 2015, 40. Komarov Botanical Institute of the Russian Academy of Sciences: St. Petersburg.

284 Smolnyakova, Yu.A., Kotelnikova, D.A. \& Zemlyanskaya, I.V. 2015a: Myxomycetes of ravine forests of Volgograd (in Russian). In: Bioticheskie svyazi gribov: mosty mezhdu tsarstvami. Materialy VII vserossiyskoy mikologicheskoy shkoly-konferentsii s mezhdunarodnym uchastiem, 2-8 avgusta $2015 \mathrm{~g}$. Sbornik dokladov i tezisov, 217-224. ZBS MSU: Moscow.

285 Smolnyakova, Yu.A., Kotelnikova, D.A. \& Zemlyanskaya, I.V. 2015b: Myxomycetes of the Chapurnikovskaya Balka Natural Monument (Volgograd region) (in Russian). In: Shepeleva, L.F. \& Yampolskaya, T.D. (eds). Sovremennye problemy botaniki, mikrobiologii i prirodopolzovaniya $v$ Zapadnoy Sibiri i na sopredelnykh territoriyakh. Materialy Vserossiyskoy nauchnoy konferentsii s mezhdunarodnym uchastiem, posvyashchennoy 10-letiyu sozdaniya kafedry botaniki i ekologii rasteniy i kafedry mikrobiologii SurGU (Surgut, 28-29 maya 2015 g.), 99-101. Publishing Center of Surgut State University: Surgut.

286 Sobolewski, G. 1799: Flora Petropolitana (in Latin). Petropoli. 
287 Sobolewski, G.F. 1802: Saint Petersburg flora (in Russian). St. Petersburg.

288 Soldatenkova, A.A. 2019: New data on myxomycetes of Yakutia and Magadan region (in Russian). In: Aleshkovskiy, I.A., Andriyanov, A.V. \& Antipov, E.A. (eds). International scientific conference of students and young scientists "Lomonosov-2019", 1. MAKS Press: Moscow.

289 Stephan, F. 1792: Enumeratio stirpium agri Mosquensis (in Latin). Mosquae.

290 Stephenson, S.L., Novozhilov, Yu.K. \& Schnittler, M. 2000: Distribution and ecology of myxomycetes in high-latitude regions of the Northern Hemisphere. Journal of Biogeography 27: 741-754.

291 Sysuev, V.A., Shirokikh, A.A. \& Shirokikh, I.G. 2014: Myxomycetes of Vyatka River Valley. In: The 8th International Congress on the Systematics and Ecology of Myxomycetes (ICSEM8). Proceedings, 22-23. Changchun, China.

292 Thümen, F. von 1878: Beiträge zur pilz-flora Sibiriens. II (in German and Latin). Bulletin de la Société Impériale des naturalistes de Moscou 53: 206-252.

293 Thümen, F. von 1880a: Beiträge zur pilz-flora Sibiriens. III (in German and Latin). Bulletin de la Société Impériale des naturalistes de Moscou 55: 72-104.

294 Thümen, F. von 1880b: Beiträge zur pilz-flora Sibiriens. IV (in German and Latin). Bulletin de la Société Impériale des naturalistes de Moscou 55: 198-233.

295 Thümen, F. von 1881: Beiträge zur pilz-flora Sibiriens. V (in German and Latin). Bulletin de la Société Impériale des naturalistes de Moscou 56: 104-134.

296 Tranzschel, V.A. 1901: List of fungi collected in Valdaysky Uyezd of Novgorod Governorate (in Russian). In: Berichte der Biologischen Süsswasserstation der Kaiserlichen Naturforscher-Gesellschaft zu St. Petersburg, Vol. 1, 160-162. Typography of V.S. Balashev and Co, St. Petersburg.

297 Tranzschel, V. 1914: Die Pilze und Myxomyceten Kamtschatka's [Fungi and myxomycetes of Kamchatka] (in Russian with German abstract). In: Elenkin, A.A. (ed). Expedition à Kamtchatka, organisée par Th. P. Riabouchinsky avec le concours de la Société Impériale Russe de Géographie. Section de Botanique. Livraison II. Plantes cryptogames de Kamtchatka: 1) algues, 2) champignons, 535-576. Typography of P.P. Ryabushinsky, Moscow.
298 Ugarov, G.S., Mikhaleva, L.G., Abramov, A.F. \& Popova, M.G. 2009: Fungi of Yakutia (in Russian). Bichik: Yakutsk.

299 Vlasenko, A.V. 2008a: Preliminary data on the myxomycetes [of] Altai krai (in Russian with English abstract). In: Dolgovykh, S.V. (ed). Biodiversity, ecological issues of Gorny Altai and its neighbouring regions: present, past, and future. Materials of International Conference held 22-26 september 2008, in Gorno-Altaisk, Russia, 205-208. Gorno-Altaisk State University: Gorno-Altaisk.

300 Vlasenko, A.V. 2008b: The history of myxomycete studies in Western Siberia (in Russian). In: Dyakov, Yu.T. (ed). Sovremennaya mikologiya v Rossii. Tom 2. Materialy 2-go Syezda mikologov Rossii, 56-57. National Academy of Mycology: Moscow.

301 Vlasenko, A.V. 2010: Myxomycetes in the pine forests on the right-bank part of the upper Ob' river (in Russian). PhD thesis. V.L. Komarov BIN RAS: St. Petersburg.

302 Vlasenko, A.V. 2011: Myxomycetes of the Tigirek Strict Nature Reserve (an annotated check-list) (in Russian with English abstract). Proceedings of the Tigirek State Natural Reserve 4: 54-56.

303 Vlasenko, A.V. 2015: Cribraria lepida in Western Siberia (in Russian with English abstract). In: Bioraznoobrazie i ekologiya gribov i gribopodobnykh organizmov severnoy Evrazii. Materialy Vserossiyskoy konferentsii s mezhdunarodnym uchastiem. Yekaterinburg, 20-24 aprelya 2005 g., 40-42. Ural University Publishing House: Yekaterinburg.

304 Vlasenko, A.V. \& Ankova, T.V. 2020: First data of the myxomycetes of the Republic of Tuva and further research prospects (in Russian with English abstract). In: Sambyla, Ch.N. (ed). Ecosystems of Central Asia: research, conservation, rational use. Materials of the XV Ubsunur International symposium (Kyzyl, July 5-8, 2020), 62-64. Ofset: Krasnoyarsk.

305 Vlasenko, A.V. \& Dulepova, N.A. 2015: First finding of Kelleromyxa fimicola in the Republic of Buryatia (Eastern Siberia). Current Research in Environmental \& Applied Mycology 5: 149-152.

306 Vlasenko, A.V. \& Novozhilov, Yu.K. 2010a: Myxomycetes of the Tigirek Strict Reserve (in Russian with English abstract). In: Mountain ecosystems of South Siberia: study, conservatin and rational nature use. The second interregional scientific-practical conference, devoted to the 10-year anniversary of the Tigirek State Natural Reserve establishment, Vol. 3, 10-12. Barnaul. 
307 Vlasenko, A.V. \& Novozhilov, Yu.K. 2010b: Rare and new for Russia myxomycete species (Myxomycetes) found in piny forests of the right-bank of the Upper Ob' river (in Russian with English abstract). Mikologiya i Fitopatologiya 44: 99-108.

308 Vlasenko, A.V. \& Novozhilov, Yu.K. 2011: Myxomycetes in the pine forests on the right-bank part of the Upper Ob' river (in Russian with English abstract). Mikologiya i Fitopatologiya 45: 465-477.

309 Vlasenko, A.V. \& Vlasenko, V.A. 2014: The first records Physarum schroeteri (Physaraceae, Myxomycetes) in the Asian Russia (in Russian with English abstract). Vestnik Novosibirskogo gosudarstvennogo universiteta. Seria biologiya i klinicheskaya medicina 12: 37-39.

310 Vlasenko, A.V. \& Vlasenko, V.A. 2017a: Ecological features and species diversity of epiphytic myxomycetes (Myxomycetes) on an ordinary pine (Pinus sylvestris L.) in the forest-steppe and steppe zones of the southeast of Western Siberia (in Russian with English abstract). Samara Journal of Science 6: 23-27.

311 Vlasenko, V.A. \& Vlasenko, A.V. 2017b: Physarum schroeteri and Polyozellus multiplex - rare Myxomycete and Aphyllophoroid fungus recommended for red data book of Novosibirsk region (in Russian with English abstract). The Bulletin of Irkutsk State University. Series: Biology and Ecology 22: 48-58.

312 Vlasenko, A.V. \& Vlasenko, V.A. 2020: First Asian record of Comatricha anomala, a rare epiphytic corticolous myxomycete. Karstenia 58: 10-15.

313 Vlasenko, A.V., Filippova, N.V. \& Vlasenko, V.A. 2018: Echinostelium novozhilovii (Echinosteliaceae, Myxomycetes), a new species from Northern Asia. Phytotaxa 367: 91-96.

314 Vlasenko, A.V., Filippova, N.V. \& Vlasenko, V.A. 2019a: Echinostelium microsporum (Echinosteliaceae, Myxomycetes), a new epiphytic corticolous species from Russia. Phytotaxa 416: 67-72.

315 Vlasenko, A.V., Novozhilov, Yu.K. \& Vlasenko, V.A. 2013a: Myxomycetes of the steppe communities plains of the Altai territory (in Russian with English abstract). Vestnik Novosibirskogo gosudarstvennogo universiteta. Seria biologiya i klinicheskaya medicina 11: 5-12.

316 Vlasenko, A.V., Novozhilov, Yu.K., Vlasenko, V.A., Shchepin, O.N., Morozova, Yu.A. \& Nikitina, A.M. 2013b: Species variety and different microhabitats (substrate types) [of] slime molds (Myxomycetes) of the tape pine forests of Altai krai (in Russian with English abstract). Vestnik Novosibirskogo gosudarstvennogo universiteta. Seria biologiya i klinicheskaya meditsina 11: 99-104.

317 Vlasenko, A.V., Novozhilov, Yu.K., Shchepin, O.N. \& Vlasenko, V.A. 2016: Hydrochory as certain mode of distribution of myxomycetes along floodlands in south of western Siberia (in Russian with English abstract). Mikologiya i Fitopatologiya 50: 14-23.

318 Vlasenko, A.V., Novozhilov, Yu.K., Vlasenko, V.A., Korolyuk, A.Yu. \& Dulepova, N.A. 2017: New data on the obligate coprophilous myxomycetes of Siberia (in Russian with English abstract). The Bulletin of Irkutsk State University. Series: Biology and Ecology 21: 50-60.

319 Vlasenko, A.V., Novozhilov, Yu.K., Prikhodko, I.S., Botyakov, V.N. \& Vlasenko, V.A. 2020: A new species of Stemonitis pseudoflavogenita from Russia, and the first record of Stemonitis capillitionodosa in Eurasia. Phytotaxa 447: 137-145.

320 Vlasenko, A.V., Vlasenko, V.A., Naumenko, Yu.V. \& Tomoschevich, M.A. 2019b: First records of rare epiphytic species Physarum lakhanpalii and Ph. lenticulare for Russia. Turczaninowia 22: 72-79.

321 Volosnova, L.F. 2014: Myxomycetes (in Russian). In: Flora of Oka Nature Reserve (vascular plants, mosses, fungi, and lichens). Proceedings of the Oka State Nature Biosphere Reserve, Vol. 30, 97-99. Golos gubernii, Ryazan.

322 Walker, G., Silberman, J.D., Karpov, S.A., Preisfeld, A., Foster, P., Frolov, A.O., Novozhilov, Yu.K. \& Sogin, M. 2003: An ultrastructural and molecular study of Hyperamoeba dachnaya, n. sp., and its relationship to the mycetozoan slime moulds. European Journal of Protistology 39: 319-336.

323 Weinmann, J.A. 1836: Hymeno- et Gastero-Mycetes hucusque in Imperio Rossico observatos (in Latin). Petropoli.

324 Wrigley de Basanta, D., Lado C. \& Estrada-Torres A. 2011: Spore to spore culture of Didymium operculatum, a new Myxomycete from the Atacama Desert of Chile. Mycologia 103: 895-903.

325 Wrigley de Basanta, D., Lado C., Garcia-Martin J.M. \& Estrada-Torres A. 2015: Didymium xerophilum, a new myxomycete from the tropical Andes. Mycologia 107: 157-168.

326 Zakharova, V.I., Kuznetsova, L.V., Ivanova, E.I. et al. 2005: Diversity of the flora of Yakutia (in Russian). Publishing House of SB RAS: Novosibirsk. 
327 Zemlyanskaya, I.V. 2003a: Myxomycetes of Bogdinsko-Baskunchakskyi Nature Reserve (in Russian with English abstract). Mikologiya i Fitopatologiya 37: 40-47.

328 Zemlyanskaya, I.V. 2003b: Myxomycetes of steppes and deserts of Lower Volga region (in Russian). PhD thesis. Volgograd State Medical University, V.L. Komarov BIN RAS: Volgograd, St. Petersburg.

329 Zemlyanskaya, I.V. 2015: On practicability of inclusion of slime moulds in the Red Lists (in Russian). In: Vedenie regionalnykh Krasnykh knig: dostizheniya, problemy i perspektivy. Sbornik materialov II Vserossiyskoy nauchno-prakticheskoy konferentsii s mezhdunarodnym uchastiem, Volgograd, 21-24 aprelya 2015 g., 14-20. Kruton: Volgograd

330 Zemlyanskaya, I.V. 2017a: Criteria for myxomycetes being qualified as rare and declining species (in Russian). In: Vedenie regionalnykh Krasnykh knig: dostizheniya, problemy i perspektivy. Sbornik materialov III Vserossiyskoy nauchno-prakticheskoy konferentsii s mezhdunarodnym uchastiem, Volgograd, 25-28 oktyabrya 2017 g., 3-6. Kruton: Volgograd.

331 Zemlyanskaya, I.V. 2017b: Monitoring of population size of rare myxomycete species included in the regional Red Lists (in Russian). In: Vedenie regionalnykh Krasnykh knig: dostizheniya, problemy i perspektivy. Sbornik materialov III Vserossiyskoy nauchno-prakticheskoy konferentsii s mezhdunarodnym uchastiem, Volgograd, 25-28 oktyabrya 2017 g., 62-68. Kruton: Volgograd.

332 Zemlyanskaya (Zemlianskaia), I.V. \& Novozhilov, Yu.K. 2010: Myxomycetes from the salt-domes near Elton lake (in Russian with English abstract). Mikologiya i

Fitopatologiya 44: 516-523.

333 Zemlyanskaya, I. \& Novozhilov, Yu. 2012: Myxomycetes (in Russian with English abstract). In: Safronova, I., Buharitsin, P. \& Barmin, A. (eds). Condition and long-term changes of natural environment in Bogdinsko-Baskunchaksky Reserve, 155-163. IPK Tsaritsyn, Volgograd.

334 Zemlyanskaya, I.V. \& Novozhilov, Yu.K. 2017: Myxomycetes (in Russian). In: Baranova, O.G. \& Sagalaev, V.A. (eds). Red Data Book of the Volgograd region. Vol. 2. Plants and other organisms, 236-249. Izdat-Print, Voronezh.

335 Zemlyanskaya, I.V. \& Rebriev, Yu.A. 2008: Myxomycetes (in Russian). In: Popov, E.S. \& Rebriev, Yu.A. (eds). IX Rabochee soveshchanie Komissii po izucheniyu makromitsetov (Vyoshenskaya, 4-10 oktyabrya 2006 g.). Annotirovannye spiski vidov gribov i miksomitsetov. Sbornik statey, 58-60. Rostov-on-Don.
336 Zemlyanskaya, I.V. \& Yanitskaya, A.V. 1999: Complex of myxomycete species formed in undisturbed phytocoenoses of the city of Volgograd (in Russian). Vestnik Volgogradskoy meditsinskoi akademii 55: 71-73.

337 Zemlyanskaya, I.V., Novozhilov, Yu.K., Smol'nyakova, Yu.A. \& Kurbatova, M.Ye. 2018: New finds of Myxomycetes of the Nature Park Volgo-Akhtuba floodplain (in Russian with English abstract). Proceedings of Voronezh State University. Series: Chemistry. Biology. Pharmacy 2: 129-134.

338 Zemlyanskaya, I.V., Smolnyakova, Yu.A., Kotelnikova, D.A. \& Novozhilov, Yu.K. 2016: Myxomycetes of the Nature Park "Volgo-Akhtuba Bottomland" (in Russian with English abstract). Mikologiya i Fitopatologiya 50: 347-353.

339 Zhulidov, D.A., Robarts, R.D., Zhulidov, A.V., Zhulidova, O.V., Markelov, D.A., Rusanov, V.A. \& Headley, J.V. 2002: Zinc accumulation by the slime mold Fuligo septica (L.) Wiggers in the former Soviet Union and North Korea. Journal of Environmental Quality 31: 1038-1042. 